\title{
Ester von (Hydroxymethyl)diorganylsilanen: Synthese und thermisch induzierte Umlagerung
}

\author{
Reinhold Tacke*a, Frank Wiesenberger ${ }^{\natural}$, Beate Beckera, Regine Rohr-Aehle ${ }^{a}$, Petra B. Schneider ${ }^{b}$, Uwe Ulbrich ${ }^{b}$, \\ Stefan M. Sarge ${ }^{b}$, Heiko K. Cammenga ${ }^{b}$, Thorsten Koslowski ${ }^{b}$ und Wolfgang von Niessen ${ }^{b}$ \\ Institut für Anorganische Chemie der Universität Karlsruhe ${ }^{\natural}$, \\ Engesserstraße, Geb. 30.45, W-7500 Karlsruhe 1 \\ Institut für Physikalische und Theoretische Chemie der Technischen Universität Braunschweig ${ }^{b}$, \\ Hans-Sommer-Straße 10, W-3300 Braunschweig \\ Eingegangen am 10. September 1991
}

Key Words: (Hydroxymethyl)diorganylsilanes, esters of / Rearrangement, thermally induced / Kinetics / DSC / Calculations, ab initio

\begin{abstract}
Esters of (Hydroxymethyl)diorganylsilanes: Synthesis and Thermally Induced Rearrangement
Twenty silanes of the type $\mathrm{R}^{1} \mathrm{R}^{2} \mathrm{Si}(\mathrm{H}) \mathrm{CH}_{2} \mathrm{OR}^{3}$ (A) were synthesized $\left[\mathrm{R}^{1}, \mathrm{R}^{2}=\mathrm{Me}, \mathrm{Ph}\right.$, 1-naphthyl, $\mathrm{PhCH}_{2}, \mathrm{Me}_{3} \mathrm{SiCH}_{2} \mathrm{OR}^{3}$ $=\mathrm{OC}(\mathrm{O}) \mathrm{Me}, \mathrm{OC}(\mathrm{O}) \mathrm{Ph}, \mathrm{OC}(\mathrm{O}) \mathrm{CF}_{3}, \mathrm{OS}(\mathrm{O})_{2} \mathrm{CF}_{3}, \mathrm{OP}(\mathrm{O}) \mathrm{Ph}_{2}$, $\mathrm{OC}(\mathrm{O}) \mathrm{Cl}]$ and studied for their thermal behaviour. The silanes $A$ undergo a thermally induced rearrangement to give the corresponding silanes $\mathrm{R}^{1} \mathrm{R}^{2} \mathrm{Si}\left(\mathrm{OR}^{3}\right) \mathrm{Me}(\mathrm{B})$. For compounds with $\mathrm{OR}^{3}=\mathrm{OC}(\mathrm{O}) \mathrm{Cl}$, an additional decarboxylation takes place to yield the chlorosilanes $R^{1} R^{2} S i(C l) M e$. Except for the derivatives with $\mathrm{OR}^{3}=\mathrm{OC}(\mathrm{O}) \mathrm{Cl}$, the energetic (reaction enthalpy)

and entropy of activation) of these reactions were studied by means of differential scanning calorimetry (DSC). In addition the kinetics of all reactions were investigated by ${ }^{1} \mathrm{H}-\mathrm{NMR}$ spectroscopy. The transition state of the rearrangement was investigated by an ab initio study based on the model compound $\left.\mathrm{H}_{3} \mathrm{SiCH}_{2} \mathrm{OC}(\mathrm{O}) \mathrm{H} \mapsto \mathrm{MeH}_{2} \mathrm{SiOC}(\mathrm{O}) \mathrm{H}\right]$. The theoretical date and the experimentally obtained energetic and kinetic data are discussed in terms of mechanistic aspects of the rearrangement reaction $\mathbf{A} \rightarrow \mathbf{B}$.
\end{abstract} and kinetic data (reaction order, frequency factor, enthalpy
In vorangegangenen Mitteilungen konnten wir zeigen, daß Silane des Typs A einer thermisch induzierten Umlagerung zu Silanen des Typs B unterliegen (Schema 1) ${ }^{[1]}$. Diese Reaktion beinhaltet eine 1,2-Wasserstoff-Verschiebung sowie eine gegenläufige 1,2-Wanderung eines von einer Elementsauerstoffsäure $\mathrm{HOR}^{3}$ abzuleitenden Säurerestes $\mathrm{OR}^{3}$. Handelt es sich bei $\mathbf{A}$ um ein Kohlensäureester-Derivat, kann sich an die Umlagerungsreaktion prinzipiell noch eine rasche Decarboxylierung anschließen ${ }^{[1 b]}$.

Schema 1

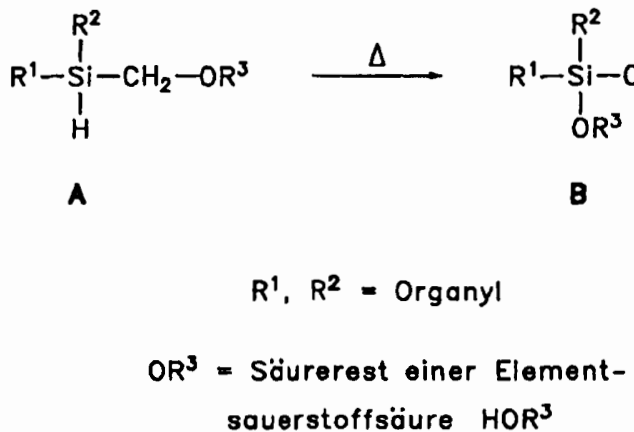

Hatten wir bisher vor allem über präparative Aspekte und erste Ergebnisse kinetischer Untersuchungen berichtet, beschreiben wir nun hier die Synthese weiterer Silane des Typs A und stellen ein umfangreicheres kinetisches Datenmaterial vor, das weitergehende Aussagen über die Struktur-Reaktivitäts-Beziehungen und eine tiefergehende mechanistische Interpretation der Umlagerungsreaktion $\mathbf{A} \rightarrow \mathbf{B}$ erlaubt. Wir berichten über die Ergebnisse kinetischer Untersuchungen $\left({ }^{1} \mathrm{H}-\mathrm{NMR}\right.$ - und DSC-Analysen) zur Umlagerung der Silane $1 \mathrm{a}-15 \mathrm{a}(\rightarrow 1 \mathrm{~b}-15 \mathrm{~b})$ (Schema 2$),[D]-11 \mathrm{a}(\rightarrow[\mathrm{D}]$ 11 b) (Schema 3) und 16a $(\rightarrow 16 b)$ (Schema 4$)$.

Außerdem werden die Ergebnisse kinetischer Experimente zur thermisch induzierten Umwandlung der Silane $17 \mathrm{a}-20 \mathrm{a}$ vorgestellt, die nach der Umlagerung zu 17b-20b sehr rasch decarboxylieren $(\rightarrow 17 c-20 c)$ (Schema 5).

Darüber hinaus berichten wir über die Ergebnisse theoretischer Untersuchungen zur Umlagerung der Modellverbindung 21 a $(\rightarrow 21$ b) (Schema 6$)$.

\section{Synthesen}

Úber die Darstellung der Silane $1 \mathrm{a}-3 \mathrm{a}^{[1 \mathrm{a}]}, 11 \mathrm{a}^{[1 \mathrm{c}]}, 12 \mathrm{a}^{[1 \mathrm{c}]}$ und $20 \mathrm{a}^{[1 \mathrm{e}]}$ haben wir bereits berichtet; die Synthese der Silane $4 a-10 a, 13 a-19 a$ und [D]-11 a wird hier erstmalig beschrieben.

Die Darstellung des (Acetoxymethyl)hydridosilans 4a erfolgte - ausgehend von dem bereits bekannten (Chlormethyl)dimethoxymethylsilan ${ }^{[2]}(22)$ - durch eine vierstufige Synthese (Schema 7). Durch Umsetzung von 22 mit (1Naphthyl)magnesiumbromid wurde zunächst das entsprechende (1-Naphthyl)silan 23 hergestelit, welches dann mit 
Schema 2<smiles>[R]C[SiH]([R1])[R]</smiles><smiles>C=CC</smiles>

$10-150$

\begin{tabular}{|c|c|c|c|}
\hline & $R^{1}$ & $\mathrm{R}^{2}$ & $O R^{3}$ \\
\hline $1 a, b$ & $\mathrm{CH}_{3}$ & $\mathrm{CH}_{3}$ & $\mathrm{OC}(0) \mathrm{CH}_{3}$ \\
\hline $2 a, b$ & $\mathrm{CH}_{3}$ & $\mathrm{C}_{6} \mathrm{H}_{5}$ & $\mathrm{OC}(0) \mathrm{CH}_{3}$ \\
\hline $3 a, b$ & $\mathrm{C}_{6} \mathrm{H}_{5}$ & $\mathrm{C}_{6} \mathrm{H}_{5}$ & $\mathrm{OC}(0) \mathrm{CH}_{3}$ \\
\hline $40, b$ & $\mathrm{CH}_{3}$ & 1-Naphthyl & $\mathrm{OC}(\mathrm{O}) \mathrm{CH}_{3}$ \\
\hline $5 a, b$ & $\mathrm{CH}_{3}$ & $\mathrm{C}_{6} \mathrm{H}_{5} \mathrm{CH}_{2}$ & $\mathrm{OC}(0) \mathrm{CH}_{3}$ \\
\hline $6 a, b$ & $\mathrm{CH}_{3}$ & $\left(\mathrm{CH}_{3}\right)_{3} \mathrm{SiCH}_{2}$ & $\mathrm{OC}(\mathrm{O}) \mathrm{CH}_{3}$ \\
\hline $7 a, b$ & $\left(\mathrm{CH}_{3}\right)_{3} \mathrm{SiCH}_{2}$ & $\left(\mathrm{CH}_{3}\right)_{3} \mathrm{SiCH}_{2}$ & $\mathrm{OC}(\mathrm{O}) \mathrm{CH}_{3}$ \\
\hline $80, b$ & $\mathrm{CH}_{3}$ & 1 -Naphthyl & $\mathrm{OC}(\mathrm{O}) \mathrm{C}_{6} \mathrm{H}_{5}$ \\
\hline $9 a, b$ & $\mathrm{C}_{6} \mathrm{H}_{5}$ & $\mathrm{C}_{6} \mathrm{H}_{5}$ & $\mathrm{OC}(0) \mathrm{C}_{6} \mathrm{H}_{5}$ \\
\hline $100, b$ & $\mathrm{C}_{6} \mathrm{H}_{5}$ & $\mathrm{C}_{6} \mathrm{H}_{5}$ & $\mathrm{OC}(0) \mathrm{CF}_{3}$ \\
\hline $11 a, b$ & $\mathrm{CH}_{3}$ & $\mathrm{CH}_{3}$ & $\mathrm{OS}(\mathrm{O})_{2} \mathrm{CF}_{3}$ \\
\hline $12 a, b$ & $\mathrm{CH}_{3}$ & $\mathrm{C}_{6} \mathrm{H}_{5}$ & $\mathrm{OS}(0)_{2} \mathrm{CF}_{3}$ \\
\hline $130, b$ & $\mathrm{C}_{6} \mathrm{H}_{5}$ & $\mathrm{C}_{6} \mathrm{H}_{5}$ & $\mathrm{OS}(0)_{2} \mathrm{CF}_{3}$ \\
\hline $140, b$ & $\mathrm{CH}_{3}$ & $\mathrm{CH}_{3}$ & $\operatorname{OP}(0)\left(\mathrm{C}_{6} \mathrm{H}_{3}\right)_{2}$ \\
\hline $15 a, b$ & $\mathrm{C}_{8} \mathrm{H}_{5}$ & $\mathrm{C}_{6} \mathrm{H}_{5}$ & $O P(O)\left(C_{6} H_{5}\right)_{2}$ \\
\hline
\end{tabular}

Schema 3<smiles>[2H][Si](C)(C)C[SeH2]O[Mg]</smiles><smiles>C=CC</smiles>

[D]-110<smiles>[R]O[Si]([R1])([R])C[2H]</smiles>

$1 b-15 b$

Schema 4<smiles>CCC(C[SiH2]C)[SiH](CC)CC(C)=O</smiles>

$16 a$

$\Delta$<smiles>CC[Si](CC)(C[SiH3])C[SiH3]</smiles>

$16 b$

Schema 5

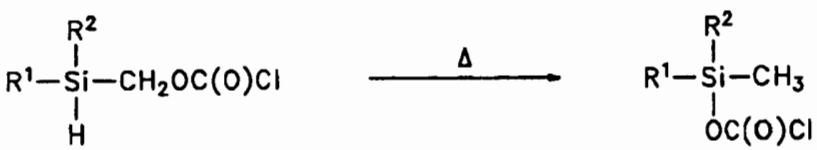

$17 a-20 a \quad 17 b-20 b$

\begin{tabular}{l|ll} 
& $\mathrm{R}^{1}$ & $\mathrm{R}^{2}$ \\
\hline $17 a, b, c$ & $\mathrm{CH}_{3}$ & $\mathrm{C}_{6} \mathrm{H}_{3} \mathrm{CH}_{2}$ \\
$18 a, b, c$ & $\mathrm{CH}_{3}$ & $\left(\mathrm{CH}_{3}\right)_{3} \mathrm{SiCH}_{2}$ \\
$19 a, b, c$ & $\left(\mathrm{CH}_{3}\right)_{3} \mathrm{SiCH}_{2}$ & $\left(\mathrm{CH}_{3}\right)_{3} \mathrm{SiCH}_{2}$ \\
$20 a, b, c$ & $\mathrm{H}$ & $\left(\mathrm{CH}_{3}\right)_{3} \mathrm{SiCH}_{2}$
\end{tabular}<smiles>CC(C)(C)C</smiles><smiles>[R][Si]([R1])(C)Cl</smiles>

$17 c-20 c$

Natriumacetat in siedendem Dimethylformamid in das (Acetoxymethyl)silan 24 übergeführt wurde. Dessen Umsetzung mit Lithiumaluminiumhydrid - gefolgt von einer Aufarbeitung mit Salzsäure - ergab das (Hydroxymethyl)hydridosilan 25, dessen Reaktion mit Essigsäureanhydrid dann zu 4a führte (Gesamtausbeute $31 \%$ ).

Das (Acetoxymethyl)hydridosilan 5a wurde - ausgehend von käuflichem Dichlor(chlormethyl)methylsilan (26) durch eine vierstufige Synthese erhalten (Schema 8). Durch Umsetzung von 26 mit Benzylmagnesiumchlorid stellte man zunächst das entsprechende Benzylsilan 27 her, welches dann mit Essigsäure/Triethylamin in siedendem Toluol in das Acetoxy(acetoxymethyl)silan 28 übergeführt wurde. Dessen Umsetzung mit Lithiumaluminiumhydrid - gefolgt von einer Aufarbeitung mit Salzsäure - ergab das entspre-

Schema 6

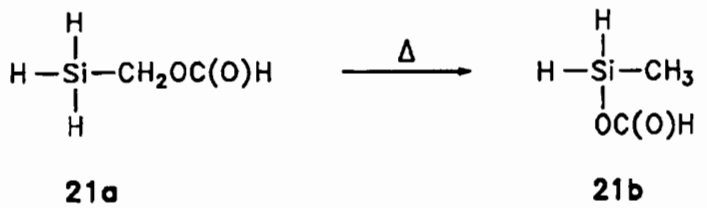

chende (Hydroxymethyl)hydridosilan 29, welches dann mit Essigsäureanhydrid in $\mathbf{5 a}$ übergeführt wurde (Gesamtausbeute $26 \%$ ).

Die (Acetoxymethyl)hydridosilane 6a und 7a wurden ausgehend von Dichlor(chlormethyl)methylsilan (26) bzw. Dichlor(chlormethyl)[(trimethylsilyl)methyl] $\operatorname{silan}^{[1 e]}$ (30) durch eine jeweils vierstufige Synthese dargestellt (Schema 9). Durch Umsetzung von 26 oder 30 mit [(Trimethylsilyl)methyl]magnesiumchlorid wurden zunächst die entsprechenden [(Trimethylsilyl)methyl]silane 31 bzw. 32 hergestellt, welche dann mit Essigsäure/Triethylamin in siedendem Toluol in die Acetoxy(acetoxymethyl)silane 33 bzw. 34 übergeführt wurden. Deren Umsetzung mit Lithiumalumi- 
Schema 7

$\mathrm{OCH}_{3}$
1
$\mathrm{CH}_{3}-\stackrel{\mathrm{Si}}{1}-\mathrm{CH}_{2} \mathrm{Cl}$
$\stackrel{\mathrm{O}}{\mathrm{C} \mathrm{H}_{3}}$

22<smiles>C[SiH](CO)c1cccc2ccccc12</smiles>

25 $\left[\mathrm{CH}_{3} \mathrm{C}(\mathrm{O})\right]_{2} \mathrm{O}$<smiles>CC(=O)OC[SiH](C)c1cccc2ccccc12</smiles>

40

Schema 8<smiles>C[Si](Cl)(Cl)CCl</smiles>

26

1. $\mathrm{LiAlH}_{4}$

2. $\mathrm{H}_{2} \mathrm{O} / \mathrm{H}_{3} \mathrm{O}^{+}$<smiles>CO[Si](C)(COC(C)=O)c1cccc2ccccc12</smiles>

24

23

$\mathrm{NoCH}_{3} \mathrm{COO}$<smiles>C[Si](Cl)(CCl)Cc1ccccc1</smiles>

27

$\mathrm{CH}_{3} \mathrm{COOH}$ $\mathrm{N}\left(\mathrm{C}_{2} \mathrm{H}_{5}\right)_{3}$<smiles>C[SiH](CO)Cc1ccccc1</smiles>

1. $\mathrm{LAIH}_{4}$

2. $\mathrm{H}_{2} \mathrm{O} / \mathrm{H}_{3} \mathrm{O}^{+}$<smiles>CC(=O)OC(C)=O</smiles>

28 $\left[\mathrm{CH}_{3} \mathrm{C}(\mathrm{O})\right]_{2} \mathrm{O}$<smiles>CC(=O)OC[Si](C)([18O])Cc1ccccc1</smiles>

Chem. Ber. 1992, 125, 591-605 niumhydrid - gefolgt von einer Aufarbeitung mit Salzsäure - ergab die entsprechenden (Hydroxymethyl)hydridosilane 35 und 36, welche dann mit Essigsäureanhydrid in $6 \mathrm{a}$ bzw. 7a übergeführt wurden (Gesamtausbeute 30 bzw. 34\%).

Schema 9<smiles>[R][Si](Cl)(Cl)CCl</smiles>

26,30<smiles>[R][Si]([R])(Cl)CCl</smiles>

31,32

$\mathrm{CH}_{3} \mathrm{COOH}$ $\mathrm{N}\left(\mathrm{C}_{2} \mathrm{H}_{5}\right)_{3}$

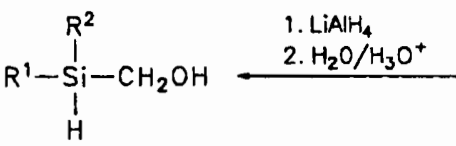

35,36<smiles></smiles>

33.34 $\left[\mathrm{CH}_{3} \mathrm{C}(\mathrm{O})\right]_{2} \mathrm{O}$

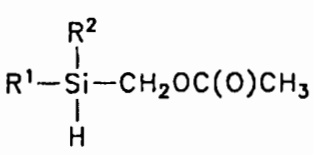

$6 a, 7 a$

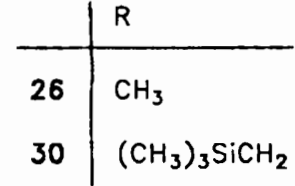

$30 \quad\left(\mathrm{CH}_{3}\right)_{3} \mathrm{SiCH}_{2}$

\begin{tabular}{l|ll} 
& $R^{\prime}$ & $R^{2}$ \\
\hline $60,31,33,35$ & $\mathrm{CH}_{3}$ & $\left(\mathrm{CH}_{3}\right)_{3} \mathrm{SiCH}_{2}$ \\
$7 \mathrm{a}, 32,34,36$ & $\left(\mathrm{CH}_{3}\right)_{3} \mathrm{SiCH}_{2}$ & $\left(\mathrm{CH}_{3}\right)_{3} \mathrm{SiCH}_{2}$
\end{tabular}

Die (Benzoyloxymethyl)hydridosilane 8a und 9a wurden gemäß Schema 10 durch Umsetzung von (Hydroxymethyl)methyl(1-naphthyl)silan (25) bzw. (Hydroxymethyl)diphenylsilan ${ }^{[3]}$ (37) mit Benzoylchlorid/Triethylamin hergestellt (Ausbeute 81 bzw. 91\%).

Schema 10

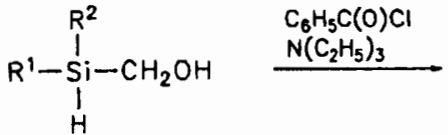

25,37<smiles>[R][Si]([R])([R])COC(=O)OCC</smiles>

80,90

\begin{tabular}{l|ll} 
& $R^{1}$ & $R^{2}$ \\
\hline 80,25 & $\mathrm{CH}_{3}$ & 1 -Naphthyl \\
90,37 & $\mathrm{C}_{6} \mathrm{H}_{5}$ & $\mathrm{C}_{6} \mathrm{H}_{5}$
\end{tabular}


Das (Trifluoracetoxymethyl)hydridosilan 10a und das (Trifluormethylsulfonyloxymethyl)hydridosilan 13a wurden entsprechend Schema 11 durch Umsetzung von (Hydroxymethyl)diphenylsilan ${ }^{[3]}$ (37) mit Trifluoressigsäureanhydrid bzw. Trifluormethansulfonsäureanhydrid in Gegenwart von Triethylamin dargestellt (Ausbeute 95 bzw. 50\%).

Schema 11<smiles>[CH][Si](CO)(c1ccccc1)c1ccccc1</smiles>

37

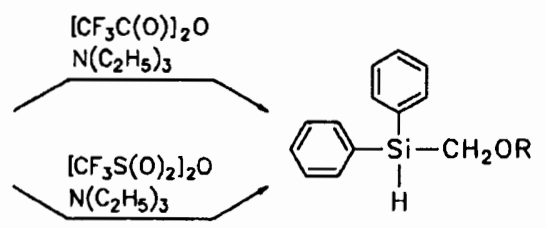

100,130

$$
\begin{array}{l|l} 
& R \\
\hline 100 & C(0) C F_{3} \\
130 & S(0)_{2} C F_{3}
\end{array}
$$

Die Darstellung der (Diphenylphosphinyloxymethyl)hydridosilane 14a und 15a erfolgte gemäß Schema 12 durch Umsetzung von (Hydroxymethyl)dimethylsilan ${ }^{[3]}$ (38) bzw. (Hydroxymethyl)diphenylsilan ${ }^{[3]}$ (37) mit Diphenylphosphinsäurechlorid/Triethylamin (Ausbeute 70 bzw. 82\%).

Schema 12<smiles>[R][SiH]([R])CO</smiles><smiles></smiles>

38,37

140,150

\begin{tabular}{l|l} 
& $R$ \\
\hline 140,38 & $\mathrm{CH}_{3}$ \\
150,37 & $\mathrm{C}_{6} \mathrm{H}_{5}$
\end{tabular}

Das Deuterio(trifluormethylsulfonyloxymethyl)silan [D]11 a wurde gemäß Schema 13 durch Umsetzung von Deuterio(hydroxymethyl)dimethylsilan ([D]-38) mit Trifluormethansulfonsäureanhydrid/Triethylamin hergestellt (Ausbeute $71 \%$ ).

\section{Schema 13}<smiles>[2H][Si](C)(C)CO</smiles>

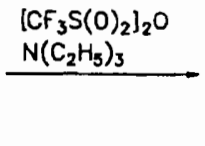

[D]-38<smiles>[2H][Si](C)(C)CO[AsH3]</smiles>

[D]-110
Das (1-Acetoxyethyl)hydridosilan 16a wurde - ausgehend von Dichlorbis[(trimethylsilyl)methyl] $\operatorname{silan}^{[4]}$ (39) - durch eine fünfstufige Synthese dargestelit (Schema 14). Durch Umsetzung von 39 mit (1-Methoxyvinyl)lithium wurde zunächst das entsprechende (1-Methoxyvinyl)silan 40 hergestellt, das dann - im Gemisch mit nicht umgesetztem 39 und dem Disubstitutionsprodukt $\left[\left(\mathrm{CH}_{3}\right)_{3} \mathrm{SiCH}_{2}\right]_{2} \mathrm{Si}-$ $\left[\mathrm{C}\left(\mathrm{OCH}_{3}\right)=\mathrm{CH}_{2}\right]_{2}-$ mit Lithiumaluminiumhydrid in das entsprechende Hydridosilan 41 übergeführt wurde. Durch Hydrolyse mit Salzsäure wurde hieraus das Acetylsilan $\mathbf{4 2}$ hergestellt. Dessen Umsetzung mit Lithiumaluminiumhydrid - gefolgt von einer Aufarbeitung mit Salzsäure - ergab das entsprechende (1-Hydroxyethyl)silan 43, welches dann mit Essigsäureanhydrid in $\mathbf{1 6 a}$ äbergeführt wurde (Gesamtausbeute $14 \%$ ).

\section{Schema 14}<smiles>[R][Si]([R])(Cl)Cl</smiles><smiles>[R][SiH]([R])C(C)=O</smiles>

42 41

1. $\mathrm{LiAlH}_{4}$

2. $\mathrm{H}_{2} \mathrm{O} / \mathrm{H}_{3} \mathrm{O}^{+}$

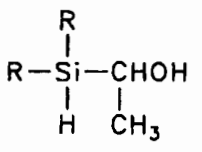

43<smiles>CC(C)(C)O[13C](C)(C)C</smiles><smiles>[R][SiH]([R])C(C)OC(C)=O</smiles>

$$
\mathrm{R}=\left(\mathrm{CH}_{3}\right)_{3} \mathrm{SiCH}_{2}
$$

Die (Chlorcarbonyloxymethyl)hydridosilane 17a-19a wurden - ausgehend von den entsprechenden (Hydroxymethyl)diorganylhydridosilanen 29, 35 bzw. 36 - gemäß Schema 15 durch Umsetzung mit Phosgen synthetisiert (Ausbeute 37, 56 bzw. 67\%).

Die durch Erhitzen der Silane 1a-20a auch im präparativen Maßstab zugänglichen Verbindungen $\mathbf{1 b}-\mathbf{1 6} \mathbf{b}$ (Schema 2 und 4) und 17c-20c (Schema 5) waren bereits bekannt [gleiche Darstellungsmethode: $\mathbf{1} b-\mathbf{3} \mathbf{b}^{[1 \mathrm{a}]}, \mathbf{1 1} \mathbf{b}^{[1 \mathrm{c}]}$, $12 b^{[1 c]}, 20 c^{[1 e]}$; andere Darstellungsmethode: $14 b^{[5]}, 17 c^{[6]}$, $\left.18 \mathrm{c}^{(7)}\right]$ oder wurden im Rahmen dieser Arbeit erstmalig synthetisiert [4b-10b, 13b, 15b, 16b, 19c]. Mit Ausnahme der ${ }^{31}$ P-NMR-spektroskopisch nicht ganz einheitlichen Verbindungen $14 b$ und $15 b$ wurden die genannten Umwandlungs- 
Schema 15

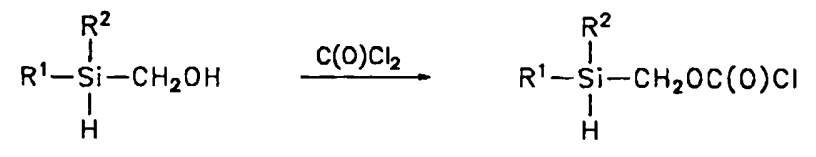

$29,35,36$

$17 a-19 a$

\begin{tabular}{l|ll} 
& $R^{1}$ & $R^{2}$ \\
\hline 170,29 & $\mathrm{CH}_{3}$ & $\mathrm{C}_{6} \mathrm{H}_{5} \mathrm{CH}_{2}$ \\
180,35 & $\mathrm{CH}_{3}$ & $\left(\mathrm{CH}_{3}\right)_{3} \mathrm{SiCH}_{2}$ \\
190,36 & $\left(\mathrm{CH}_{3}\right)_{3} \mathrm{SiCH}_{2}$ & $\left(\mathrm{CH}_{3}\right)_{3} \mathrm{SiCH}_{2}$
\end{tabular}

produkte mit nahezu quantitativer Ausbeute in NMR-spektroskopisch $\left({ }^{1} \mathrm{H},{ }^{13} \mathrm{C}\right)$ reiner Form erhalten.

\section{${ }^{1}$ H-NMR-Untersuchungen zur Kinetik}

Die Kinetik der thermisch induzierten Umwandlungen der Silane 1 a-20a wurde ${ }^{1} \mathrm{H}-\mathrm{NMR}$-spektroskopisch an ca. 0.6-0.1 M Lösungen der Substanzen in $\left[D_{6}\right]$ Benzol verfolgt. Die Umlagerung von 9a wurde außerdem an Lösungen dieser Verbindung in Cyclohexan und Aceton studiert.

Tab. 1. Mittels 'H-NMR-Spektroskopie bestimmte Halbwertszeiten $\tau_{1 / 2}$ und Geschwindigkeitskonstanten $k_{9}^{\text {NMR }}$ der thermisch induzierten Umwandlungen von $1 \mathrm{a}-20 \mathrm{a}$ in $\mathrm{C}_{6} \mathrm{D}_{6}{ }^{(\text {a) }}$ sowie Vergleich mit den durch DSC-Messungen ${ }^{[b]}$ erhaltenen Geschwindigkeitskonstanten $k_{9}^{\text {DSC }}$ der Umlagerungen von 1a-16a

\begin{tabular}{|c|c|c|c|c|}
\hline Umlagerung & $\frac{\tau_{1 / 2}}{h}$ & $\frac{\vartheta}{{ }^{0} \mathrm{C}}$ & $\frac{k_{i}^{N M R}}{10^{-5} s^{-1}}$ & $\frac{k_{i}^{D S C}}{10^{-3} s^{-1}}$ \\
\hline $1 a \rightarrow 1 b$ & $\overline{11}$ & $\overline{\overline{135}}$ & $\overline{1.8}$ & $2.70 \pm 0.25$ \\
\hline $2 a \rightarrow 2 b$ & 7.7 & 135 & 2.5 & $2.66 \pm 0.04$ \\
\hline $\mathbf{3 a} \rightarrow \mathbf{8 b}$ & 4.2 & 135 & 4.6 & $5.27 \pm 0.34$ \\
\hline $4 a \rightarrow 4 b$ & 30 & 135 & 0.6 & $1.01 \pm 0.04$ \\
\hline $5 \mathbf{a} \rightarrow 5 \mathbf{b}$ & 9.2 & 135 & 2.1 & $3.14 \pm 0.20$ \\
\hline $6 \mathbf{a} \rightarrow 6 \mathrm{~b}$ & 11 & 135 & 1.8 & $2.45 \pm 0.27$ \\
\hline $7 \mathrm{a} \rightarrow 7 \mathrm{~b}$ & 9.6 & 135 & 2.0 & $2.48 \pm 0.08$ \\
\hline $8 a \rightarrow 8 b$ & 15 & 135 & 1.3 & $1.90 \pm 0.07$ \\
\hline $8 \mathbf{a} \rightarrow 9 \mathbf{b}$ & 2.0 & 135 & 9.6 & $10.96 \pm 0.19$ \\
\hline $10 \mathrm{a} \rightarrow 10 \mathrm{~b}$ & 8.7 & 135 & 2.2 & $3.91 \pm 0.55$ \\
\hline $11 a \rightarrow 11 b$ & 0.8 & 80 & 25.7 & $27.24 \pm 0.58$ \\
\hline $12 \mathrm{a} \rightarrow 12 \mathrm{~b}$ & 1.7 & 80 & 11.3 & $12.47 \pm 0.18$ \\
\hline $13 \mathrm{a} \rightarrow 13 \mathrm{~b}$ & 3.6 & 80 & 5.3 & $6.77 \pm 0.13$ \\
\hline $14 a \rightarrow 14 b$ & 2.6 & 80 & 7.3 & $6.82 \pm 0.04$ \\
\hline $15 a \rightarrow 15 b$ & 2.4 & 60 & 8.1 & $8.57 \pm 0.27$ \\
\hline $16 a \rightarrow 16 b$ & 6.2 & 135 & 3.1 & $4.37 \pm 0.08$ \\
\hline $17 \mathrm{a} \rightarrow 17 \mathrm{c}$ & 3.2 & 135 & 6.0 & - \\
\hline $18 a \rightarrow 18 c$ & 3.6 & 135 & 5.3 & - \\
\hline $18 \mathrm{a} \rightarrow 18 \mathrm{c}$ & 2.8 & 135 & 6.8 & - \\
\hline $20 a \rightarrow 20 c$ & 3.3 & 135 & 5.8 & - \\
\hline
\end{tabular}

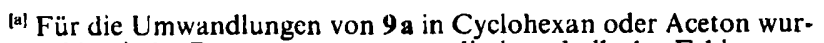
den kinetische Parameter gemessen, die innerhalb der Fehlergrenzen mit den Daten übereinstimmen, die für die Umlagerung in $C_{6} D_{6}$ bestimmt wurden. - ${ }^{[b]}$ Umwandlung in Substanz (1a-9a, $11 \mathrm{a}-13 \mathrm{a}, 16 \mathrm{a})$ oder in $C_{6} D_{6}(10 \mathrm{a}, 14 \mathrm{a}, 15 \mathrm{a})$.
Die Ergebnisse dieser Untersuchungen sind in Tab. $1 \mathrm{zu}-$ sammengefaßt und werden dort den durch DSC-Untersuchungen erhaltenen Daten gegenübcrgestellt.

\section{DSC-Untersuchungen zur Kinetik}

Die kinetischen und energetischen Parameter (Frequenzfaktor, Aktivierungsenergie, Reaktionsenthalpie) der Umlagerungsreaktionen der Silane 1a-16a und [D]-11a wurden mit Hilfe der Dynamischen Differenzkalorimetrie ${ }^{[8]}$ (Differential Scanning Calorimetry, DSC) ermittelt. Nach der Methode der Multiplen Linearen Regression (MLR) ergab sich für die Umlagerungen der untersuchten Substanzen jeweils eine Reaktionsordnung $n$ nahe $1( \pm 0.05)$. Die mit Hilfe der MLR erhaltenen Werte für den Frequenzfaktor $A$ und die Aktivierungsenergie $E_{A}$ unterscheiden sich somit nur wenig von jenen Werten, die unter Anwendung der Auswertungsmethode von Borchardt und Daniels ${ }^{[9]}$ bei Annahme einer Reaktionsordnung von $n=1$ crhalten wurden. In Tab. 2 sind daher nur die Ergebnisse der letztgenannten Auswertung (im Umsatzbereich von 10 bis $90 \%$ ) und die daraus berechneten kinetischen Daten aufgelistet; in Tab. 3 sind die gemessenen Reaktionsenthalpien $\Delta_{\mathrm{r}} H$ zusammengestellt. Angegeben sind jeweils der Mittelwert $\bar{x}$ aus $j$ bzw. $i$ Messungen ${ }^{[10]}$ und der mittlere Fehler des Mittelwertes $\sigma_{\bar{x}}$ (Details: exp. Teil und Lit. ${ }^{(1 d .11)}$. Die mittels DSC-Untersuchungen bestimmten Geschwindigkeitskonstanten für die Umlagerungen von $1 \mathbf{a}-9 \mathrm{a}, 11 \mathrm{a}-13 \mathbf{a}$ und 16a in Substanz sowie für die Umlagerungen von $10 \mathrm{a}, 14 \mathrm{a}$ und $15 \mathrm{a}$ in $\mathrm{C}_{6} \mathrm{D}_{6}$ werden in Tab. 1 den durch ${ }^{1} \mathrm{H}$-NMR-Messungen ermittelten Geschwindigkeitskonstanten für die Umlagerungen von $1 \mathrm{a}-16 \mathrm{a}$ in $\mathrm{C}_{6} \mathrm{D}_{6}$ gegenübergestellt.

Eine verläßliche Bestimmung der Reaktionsordnung und der kinetischen Parameter für die Umwandlungen der Silane $17 \mathrm{a}-20 \mathrm{a}(\rightarrow 17 \mathrm{c}-20 \mathrm{c})$ mittels DSC-Untersuchungen war nicht möglich. Die in Lit. ${ }^{(9)}$ beschriebene Auswertungsmethode ist auf diese Umwandlungen strenggenommen nicht anwendbar, weil davon auszugehen ist, da $B$ sich diese $\mathrm{Re}$ aktionen jeweils aus zwei Teilschritten (Umlagerung + Decarboxylierung) mit unterschiedlichen Geschwindigkeitskonstanten zusammensetzen. Die in Lit. ${ }^{\left[{ }^{[9]}\right.}$ beschriebene Methode liefert aber nur dann sinnvolle Ergebnisse, wenn die zweite Reaktion sehr schnell gegenüber der ersten ist. Ein weiteres Problem besteht darin, daß bei der Bildung eines gasförmigen Produktes (hier $\mathrm{CO}_{2}$ ) störendc Wärmeeffekte auftreten können.

\section{Theoretische Untersuchungen}

Die Umlagerungsreaktion $\mathbf{A} \rightarrow \mathbf{B}$ wurde am Beispiel der Modellverbindungen 21 a (Edukt) und 21 b (Produkt) und des entsprechenden Übergangszustands theoretisch untersucht. Zur Berechnung der Moleküleigenschaften wurden das semiempirische MNDO-Verfahren ${ }^{[12]}$ und das ab-initioPaket GAUSSIAN 82 ${ }^{[13]}$ benutzt (Details: exp. Teil). Die aufwendigen ab-initio-Rechnungen legten eine Beschränkung auf möglichst wenige Basisfunktionen - also auch auf möglichst wenige Atome - nahe. Die bereits erwähnten Modellverbindungen sind die kleinstmöglichen, die den experimentell untersuchten Verbindungen entsprechen. 
Tab. 2. Mittels DSC-Messungen bestimmte kinetische Parameter der Umlagerungen von 1a $-16 \mathbf{a}$ und $[D]-11 \mathbf{a}^{[a]}$

\begin{tabular}{|c|c|c|c|c|c|c|c|}
\hline Umls & $\begin{array}{l}\text { Lösungs- } \\
\text { mittel }\end{array}$ & $j$ & $\lg \left(A / s^{-1}\right)$ & $\frac{E_{A}}{\mathrm{~kJ} \mathrm{~mol}^{-1}}$ & $\frac{\Delta H_{408}^{!}}{\mathrm{kJ} \mathrm{mol}}$ & $\frac{\Delta S_{\mathrm{lng}}^{t}}{\mathrm{~J} \mathrm{~K}^{-1} \mathrm{~mol}^{-1}}$ & $\frac{\Delta G_{408}^{\mathrm{t}}}{\mathrm{kJ} \mathrm{mol}^{-1}}$ \\
\hline & & & 31 & $111.7 \pm 2.7$ & $08.3 \pm 2.7$ & $-69.9 \pm 5.9$ & $136.8 \pm 0.3$ \\
\hline $2 a \rightarrow 2 b$ & & & 10.8 & $120.49 \pm 0.83$ & $117.09 \pm 0.83$ & $-48.2 \pm 1.9$ & $136.78 \pm 0.06$ \\
\hline $\mathbf{3 a} \rightarrow \mathbf{3 b}$ & & 2 & 10.6 & $116.2 \pm 2.2$ & $112.9 \pm 2.2$ & $-52.9 \pm 4.8$ & $134.5 \pm 0.2$ \\
\hline & & & 0.22 & $116.20 \pm 0.94$ & $112.80 \pm 0.94$ & $-66.2 \pm 2.1$ & $139.84 \pm 0.10$ \\
\hline$\rightarrow \overline{5 b}$ & & & 10.3 & $116.2 \pm 1.8$ & $112.8 \pm 1.8$ & $-57.5 \pm 4.0$ & $136.2 \pm 0.2$ \\
\hline $6 a \rightarrow 6 b$ & & & 10.6 & $119.4 \pm 2.4$ & $6.1 \pm 2.4$ & $-51.5 \pm 5.0$ & $1 \pm 0.4$ \\
\hline $7 a \rightarrow 7 b$ & & 8 & 10.9 & $121.87 \pm 0.67$ & $118.48 \pm 0.67$ & $-45.5 \pm 1.4$ & $137.08 \pm 0.12$ \\
\hline & & 3 & 9.6 & $112.3 \pm 1.0$ & $108.9 \pm 1.0$ & $-71.2 \pm 2.3$ & $137.9 \pm 0.1$ \\
\hline$+9 \mathrm{~b}$ & & 4 & 10.4 & $112.4 \pm 1.1$ & $.0 \pm 1.1$ & $-56.4 \pm 2.4$ & $D \pm 0.1$ \\
\hline$\theta a \rightarrow \theta b$ & -1 & 3 & 10.0 & $109.6 \pm 1.6$ & $106.2 \pm 1.6$ & $-62.8 \pm 3.7$ & $131.9 \pm 0.1$ \\
\hline $9 \mathbf{a} \rightarrow \mathbf{9 b}$ & $\left(\mathrm{CH}_{3}\right)_{2} \mathrm{CO}$ & 3 & 10.00 & $109.2 \pm 2.0$ & $105.8 \pm 2.0$ & $-64.4 \pm 4.7$ & $132.1 \pm 0.2$ \\
\hline & & 1 & 9.60 & $109.6 \pm 1.7$ & $106.2 \pm 1.7$ & $-72.0 \pm 3.8$ & $135.6 \pm 0.4$ \\
\hline$\rightarrow 1$ & & 4 & 10.0 & $92.13 \pm 0.59$ & $88.73 \pm 0.59$ & $-63.2 \pm 1.5$ & $41 \pm 0.04$ \\
\hline$-11 a \rightarrow[D]-11$ & & 5 & 10.3 & $95.21 \pm 0.22$ & $91.82 \pm 0.22$ & $-58.16 \pm 0.58$ & $55 \pm 0.03$ \\
\hline$\rightarrow 12 b$ & & 6 & 10.79 & $99.31 \pm 0.54$ & $95.95 \pm 0.54$ & $-49.3 \pm 1.4$ & $116.07 \pm 0.04$ \\
\hline- & & 5 & 10.8 & $101.24 \pm 0.22$ & $97.85 \pm 0.22$ & $-48.99 \pm 0.59$ & $117.85 \pm 0.06$ \\
\hline $14 a \rightarrow 14 b$ & 000 & $1-$ & 11.2 & $104.5 \pm 1.0$ & $101.1 \pm 1.0$ & $-39.7 \pm 2.9$ & $117.3 \pm 0.2$ \\
\hline $15 a \rightarrow 15 b$ & & 4 & 0.23 & $101.4 \pm 1.6$ & $98.0 \pm 1.6$ & $-30.7 \pm 4.5$ & $110.5 \pm 0.2$ \\
\hline $15 a \rightarrow 15 b$ & $\mathrm{C}_{6} \mathrm{D}_{6}$ & 2 & 10.92 & $95.6 \pm 1.2$ & $92.2 \pm 1.2$ & $-46.8 \pm 3.3$ & $111.3 \pm 0.2$ \\
\hline $16 a \rightarrow 16 b$ & & - & $10.75 \pm 0.15$ & $118.1 \pm 1.3$ & $114.7 \pm 1.3$ & $-50.0 \pm 2.9$ & $135.1 \pm 0.1$ \\
\hline
\end{tabular}

[a] In jenen Fällen, in denen kein Lösungsmittel angegeben ist, beziehen sich die Daten auf die Umlagerungen der reinen Substanzen. Die Korrelationskoeffizienten für die Einzelauswertungen nach der Methode von Borchardt und Daniels ${ }^{91}$ sind in allen Fällen größer als 0.9990 .

Tab. 3. Mittels DSC-Messungen bestimmte Reaktionsenthalpien der Umlagerungen von $1 \mathrm{a}-16 \mathrm{a}$ und $[\mathrm{D}]-11 \mathbf{a}^{[\mathrm{a}]}$

\begin{tabular}{|c|c|c|c|}
\hline Umlagerung & $\begin{array}{l}\text { Lösungs- } \\
\text { mittel }\end{array}$ & 2 & $\frac{\Delta_{r} H}{\mathrm{~kJ} \mathrm{~mol}^{-1}}$ \\
\hline 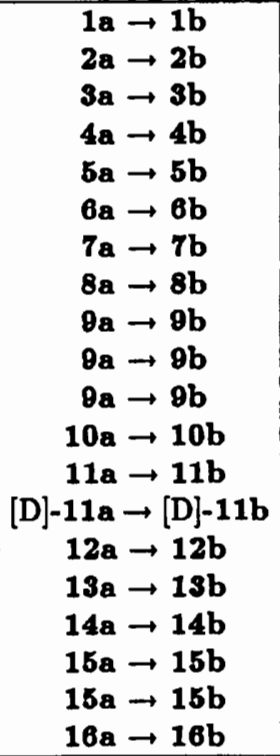 & $\begin{array}{c}\mathrm{C}_{6} \mathrm{H}_{12} \\
\left(\mathrm{CH}_{3}\right)_{2} \mathrm{CO} \\
\mathrm{C}_{6} \mathrm{D}_{6}\end{array}$ & $\begin{array}{l}1 \\
4 \\
3 \\
4 \\
4 \\
2 \\
8 \\
3 \\
4 \\
3 \\
3 \\
4 \\
4 \\
5 \\
4\end{array}$ & $\begin{array}{l}-178.2 \\
-180.3 \pm 2.7 \\
-187.4 \pm 2.3 \\
-175.5 \pm 1.3 \\
-147.9 \pm 8.6 \\
-183.4 \pm 4.3 \\
-168.1 \pm 1.6 \\
-164.1 \pm 2.6 \\
-169.1 \pm 2.8 \\
-180.7 \pm 4.3 \\
-168.6 \pm 9.8 \\
-131.6 \pm 6.9 \\
-218.0 \pm 5.8 \\
-198.0 \pm 0.5 \\
-161.4 \pm 2.2 \\
-193.4 \pm 1.3 \\
-216.5 \\
-201.3 \pm 1.3 \\
-219.2 \\
-163.1 \pm 4.3\end{array}$ \\
\hline
\end{tabular}

[a] Siehe Fußnote von Tab. 2.

Aus den mit „double-zeta“-Basissätzen berechneten Bildungsenthalpien von 21 a und 21 b ergibt sich für die Reaktionsenthalpie der Umlagerung $21 \mathbf{a} \rightarrow 21$ b ein Wert von
$\Delta_{\mathrm{r}} H=-170 \mathrm{~kJ} \mathrm{~mol}^{-1}$. Die Aktivierungsenthalpie beträgt $\Delta H^{*}=117 \mathrm{~kJ} \mathrm{~mol}^{-1}$.

Liegen die Bindungsabstände und -winkel vom Anfangsund Endzustand im Rahmen des Erwarteten, so weist die Geometrie des Úbergangszustands erhebliche Abweichungen von tabellierten Bindungslängen ${ }^{[14]}$ auf. Die Nichtwasserstoff-Atome des Übergangszustands (Schema 16) bilden einen planaren Fünfring mit der Atomsequenz $\mathrm{Si}-\mathrm{C}(1)-\mathrm{O}(1)-\mathrm{C}(2)-\mathrm{O}(2)$. Jeder Versuch, diesen Ring zu verformen, führt zu einem Anstieg der Aktivierungsenthalpie. Die Bindungswinkel innerhalb des Rings betragen $103^{\circ}$ $[\mathrm{Si}-\mathrm{C}(1)-\mathrm{O}(1)], 105^{\circ}[\mathrm{C}(1)-\mathrm{O}(1)-\mathrm{C}(2)], 119^{\circ}[\mathrm{O}(1)-$ $\mathrm{C}(2)-\mathrm{O}(2)], 122^{\circ}[\mathrm{C}(2)-\mathrm{O}(2)-\mathrm{Si}]$ und $88^{\circ}[\mathrm{O}(2)-\mathrm{Si}-$ $\mathrm{C}(1)]$. Die Bindungsabstände innerhalb des Rings ergeben sich zu $1.58 \AA[\mathrm{Si}-\mathrm{C}(1)], 2.19 \AA[\mathrm{C}(1)-\mathrm{O}(1)], 1.25 \AA$ $[\mathrm{O}(1)-\mathrm{C}(2)], 1.22 \AA[\mathrm{C}(2)-\mathrm{O}(2)]$ und $2.06 \AA[\mathrm{O}(2)-\mathrm{Si}]$.

Die Geometrie des Übergangszustands ist - bei Nichtbeachtung des Unterschieds zwischen dem Si- und C(1)Atom - beinahe symmetrisch bezüglich einer senkrecht zur Molekülebene stehenden Spiegelebene, die das C(2)-Atom und den Mittelpunkt der $\mathrm{Si}-\mathrm{C}(1)$-Bindung schneidet. $\mathrm{Zu}$ beachten sind der verkürzte $\mathrm{Si}-\mathrm{C}(1)$-Abstand sowie die ähnlich langen $\mathrm{C}(1)-\mathrm{O}(1)$ - und $\mathrm{Si}-\mathrm{O}(2)$-Bindungen. Das wandernde Wasserst off-Atom ist zwischen dem Si- und C(1)Atom lokalisiert; der $\mathrm{Si}-\mathrm{H}$-Abstand beträgt $1.84 \AA$, und der entsprechende Winkel $\mathrm{H}-\mathrm{Si}-\mathrm{C}(1)$ ergibt sich zu $71^{\circ}$. Das wandernde Wasserstoff-Atom liegt innerhalb der Ringebene etwa gleich weit vom $\mathrm{Si}$ - und $\mathrm{C}(1)$-Atom entfernt.

Im Verlauf der Geometrieoptimierung zerfällt zunächst der Fünfring [Bruch der $\mathrm{C}(1)-\mathrm{O}(1)$-Bindung und Festigung 
Schema 16

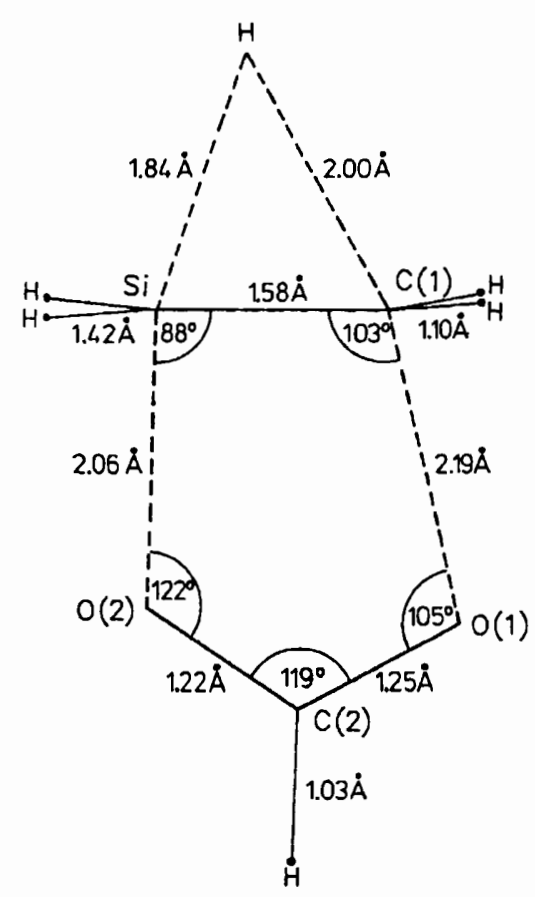

der $\mathrm{Si}-\mathrm{O}(2)$-Bindung]; erst gegen Ende der Reaktion erfolgt die 1,2-Wasserstoff-Verschiebung vom Si-zum C(1)-Atom.

\section{Zum Mechanismus der Umlagerung}

Die hier untersuchte Umlagerung $\mathbf{A} \rightarrow \mathbf{B}$ läßt sich formal dem in der Literatur ${ }^{[15]}$ an mehreren Beispielen dokumentierten Reaktionstyp $\mathrm{R}_{2}(X) \mathrm{Si}-\mathrm{C}(Y) \mathrm{R}_{2} \rightarrow \mathrm{R}_{2}(Y) \mathrm{Si}-\mathrm{C}(X) \mathrm{R}_{2}$ zuordnen, der durch eine thermisch induzierte 1,2-Verschiebung von $X$ und $Y$ im Sinne eines Substituentenaustauschs am Silicium und Kohlenstoff gekennzeichnet ist $[X / Y=$ $\mathrm{SiMe}_{3} / \mathrm{OAc}^{[15 b]} ; \mathrm{Ph} / \mathrm{F}, \mathrm{Cl}, \mathrm{Br}, \mathrm{OAc}, \mathrm{OTs}^{[15 \mathrm{~s}, \mathrm{e}, \mathrm{g}, \mathrm{l}]} ; \mathrm{Me} / \mathrm{Br}, \mathrm{OAc}$, $\left.\mathrm{OAc}_{f}, \mathrm{OP}(\mathrm{O}) \mathrm{R}_{2}{ }^{[15 e-\mathrm{g}]} ; \mathrm{Et} / \mathrm{Br}^{[15 e]} ; \mathrm{Cl} / \mathrm{F}^{[15 d]}\right]$. Die wenigen bisher durchgeführten Untersuchungen ${ }^{[15 e, g]}$ zum Mechanismus dieser Reaktion machen allerdings deutlich, daß die Zusammenfassung aller in Lit. ${ }^{[15]}$ beschriebenen Umlagerungen zu einem Reaktionstyp nur formalen Charakter haben kann, da sich die einzelnen Umlagerungen nicht durch einen einheitlichen Reaktionsmechanismus beschreiben lassen. Die von uns durch Zufall entdeckte thermisch induzierte Umlagerung $\mathbf{A} \rightarrow \mathbf{B}$ (vgl. hierzu Lit. ${ }^{[1 \mathrm{a}]}$ ) stellt mit ihrer 1,2-Wasserstoff-Verschiebung $(X=\mathrm{H})$ einen Sonderfall dar und forderte deshalb zu mechanistischen Studien heraus ${ }^{[16]}$.

Die Reaktion $\mathbf{A} \rightarrow \mathbf{B}$ zeigt allgemein eine hohe negative Reaktionsenthalpie $\Delta_{\mathrm{r}} H$ (Tab. 3). Die experimentell bestimmten Werte stimmen in den meisten Fällen sehr gut mit dem für die Modellreaktion $21 \mathbf{a} \rightarrow \mathbf{2 1} \mathbf{b}$ berechneten Wert von $\Delta_{\mathrm{r}} H=-170 \mathrm{~kJ} \mathrm{~mol}^{-1}$ überein. Gleiches gilt auch für die experimentell bestimmten Aktivierungsenthalpien und den für das Modellsystem berechneten Wert von $\Delta H^{*}=$ $117 \mathrm{~kJ} \mathrm{~mol}^{-1}$.

Die kinetischen Experimente ergaben, daß die Silane $1 \mathrm{a}-20$ a gemäß einer Kinetik 1 . Ordnung umlagern, wobei Unterschiede im Frequenzfaktor um mehr als zwei Größen- ordnungen beobachtet wurden (Tab. 1 und 2). Für die mittels ' $\mathrm{H}$-NMR- und DSC-Untersuchungen bestimmten Geschwindigkeitskonstanten wurde zumeist eine recht gute Übereinstimmung gefunden (Tab. 1) ${ }^{[17]}$. Auffällig ist, daß vor allem der Säurerest $\mathrm{OR}^{3}$ die Reaktionsgeschwindigkeit beeinflußt, während die an das Silicium-Atom gebundenen Substituenten $\mathbf{R}^{1}$ und $\mathbf{R}^{2}$ - abgesehen von dem die Umlagerung erschwerenden 1-Naphthyl-Rest - nur wenig Einfluß haben. Die Umlagerung der Silane 11a-15a (Trillate und Phosphinate) erfolgt schon unter so milden Bedingungen, daß die Gewinnung reiner Präparate dieser Verbindungen bereits zu einem Problem wird.

Durch Kreuzungsexperimente mit 2a und dem von 3a abzuleitenden Isotopomer $\mathrm{Ph}_{2} \mathrm{Si}(\mathrm{D}) \mathrm{CH}_{2} \mathrm{OAc}$ konnten wir bereits früher für die Umlagerung dieser Silane eine intramolekulare 1,2-Wasserstoff-Verschiebung nachweisen ${ }^{[1]}$. $\mathrm{Da}$ der für die Umlagerung von 11 a und [D]-11a beobachtete kinetische Isotopeneffe $k t\left[k_{\mathrm{H}} / k_{\mathrm{D}}=1.55\right.$; berechnet aus den entsprechenden Werten für $k_{80}^{\mathrm{DSC}}$ : $(27.24 \pm 0.58)$ $10^{-5} \mathrm{~s}^{-1}$ (11 a) bzw. (17.54 \pm 0.14$) 10^{-5} \mathrm{~s}^{-1}$ ([D]-11 a)] vergleichsweise klein ist, kann die 1,2-Wasserstoff-Verschiebung als geschwindigkeitsbestimmender Schritt ausgeschlossen werden. Unter Einbeziehung der experimentell bestimmten Reaktionsordnung $(n=1)$ läßt sich damit auch auf eine intramolekulare 1,2-Wanderung des Säurerestes $\mathrm{OR}^{3}$ schließen.

Die Tatsache, daß die Umlagerungsgeschwindigkeit praktisch unabhängig von der Polarität des verwendeten Lösungsmittels ist (Umlagerung von 9a in Substanz, in Cyclohexan und in Aceton; Tab. 2), läßt einen ionischen Mechanismus unter Beteiligung von Kontakt-Ionenpaaren unwahrscheinlich erscheinen. Die hohe negative Aktivierungsentropie $\Delta S_{408}^{*}$ der Umlagerung (Tab. 2) legt vielmehr die Annahme eines hochgeordneten cyclischen Übergangszustands nahe, wie er auch aus den theoretischen Untersuchungen folgt (Schema 16). Ein weiteres experimentelles Indiz für einen derartigen Ưbergangszustand ist die hohe Beschleunigung der Reaktion durch Druck, wie sie am Beispiel der Umlagerung 9a $\rightarrow$ 9b nachgewiesen wurde ${ }^{[18]}$.

Auf der Basis der hier vorgestellten experimentellen und theoretischen Untersuchungen schlagen wir für die Umlagerung $\mathbf{A} \rightarrow \mathbf{B}$ den folgenden Mechanismus vor: Im ersten Schritt der Reaktion kommt es zur Ausbildung eines hochgeordneten cyclischen Übergangszustands mit einem pentakoordinierten Silicium-Atom ${ }^{[19]}$, wie er in Schema 16 für die Reaktion der Modellverbindung 21 a skizziert ist. Dieser Übergangszustand zerfällt unter Aufbau der neuen $\mathrm{Si}-\mathrm{O}$ Bindung und Spaltung der C-O-Bindung, worauf sich schließlich eine rasche 1,2-Wasserstoff-Verschiebung anschließt. Diese Vorstellungen decken sich weitgehend mit dem Modell, das für die Umlagerung $\mathrm{Me}_{2} \mathrm{PhSi}-\mathrm{C}(\mathrm{OAc}) \mathrm{Ph}_{2}$ $\rightarrow \mathrm{Me}_{2}(\mathrm{OAc}) \mathrm{Si}-\mathrm{CPh}_{3}$ entwickelt wurde ${ }^{[15 \mathrm{~g}]}$. Bei der letztgenannten Reaktion führt der Austausch des Acetat- durch den Trifluoracetat-Rest allerdings zu einer starken Reaktionsbeschleunigung, während wir selbst bei einem Acetat/ Trifluoracetat-Austausch ( $\mathbf{3}$ a/10a) eine kleine Verringerung der Reaktionsgeschwindigkeit beobachtet haben (Tab. 2). Dies muß aber kein Widerspruch sein, denn bereits die Da- 
ten in Tab. 2 machen deutlich, daß die Effekte der einzelnen Substituenten $\mathbf{R}^{1}, \mathbf{R}^{2}$ und $\mathrm{OR}^{3}$ auf die Reaktionsgeschwindigkeit nicht isoliert betrachtet werden dürfen, sondern daß diese Substituenteneffekte selbst vielmehr von den jeweils anderen Substituenten mit beeinflußt werden.

Diese Untersuchungen wurden durch Mittel der Deutschen Forschungsgemeinschaft und des Fonds der Chemischen Industrie sowie durch Chemikalienspenden der Bayer $A G$ (Leverkusen und Wuppertal-Elberfeld) unterstützt. Den Herren Prof. Dr. F.-G. Klärner und Dipl.-Chem. D. Hochstrate, Universität Bochum, danken wir für die Durchführung der Umlagerungsexperimente bei hohem Druck.

\section{Experimenteller Teil}

a) Synthesen: Alle Reaktionen wurden unter Schutzgas (trockener Stickstom) durchgeführt; die verwendeten Lösungsmittel waren wasserfrei (Siedebereich des Petrolethers $40-70^{\circ} \mathrm{C}$ ). - Schmelzpunkte: Schmelzpunktbestimmungsgerät der Fa. Büchi, Typ 530. - ${ }^{1} \mathrm{H}$ NMR: WM-400- (400.1 MHz), AM-400- (400.1 MHz), AM-300(300.1 MHz) oder AC-200-Gerät (200.1 MHz) der Fa. Bruker. Lösungsmittel $\mathrm{C}_{6} \mathrm{D}_{6}$ bzw. $\mathrm{CDCl}_{3}$; interner Standard $\mathrm{C}_{6} \mathrm{D}_{5} \mathrm{H}(\delta=7.15)$, $\mathrm{CHCl}_{3}(\delta=7.25)$ oder TMS $(\delta=0)$. $-{ }^{13} \mathrm{C}-\mathrm{NMR}$ : WM-400- $(100.6$ MHz), AM-400- (100.6 MHz), AM-300- (75.5 MHz) oder AC-200Gerät $(50.3 \mathrm{MHz})$ der Fa. Bruker. Lösungsmittel und interner Standard $\mathrm{C}_{6} \mathrm{D}_{6}(\delta=128.0)$ oder $\mathrm{CDCl}_{3}(\delta=77.05)$. Die Signalzuordnungen wurden durch DEPT-Experimente unterstützt; die Ergebnisse dieser Messungen sind in den Zuordnungen enthalten. $-{ }^{29} \mathrm{Si}$ NMR: AC-200-Gerät $(39.8 \mathrm{MHz})$ der Fa. Bruker. Lösungsmittel $\mathrm{C}_{6} \mathrm{D}_{6}$ oder $\mathrm{CDCl}_{3}$; interner Standard TMS $(\delta=0)$. - ${ }^{31} \mathrm{P}-\mathrm{NMR}$ AC-200-Gerät $(81.0 \mathrm{MHz})$ der Fa. Bruker. Lösungsmittel $\mathrm{C}_{6} \mathrm{D}_{6}$ externer Standard 85proz. $\mathrm{H}_{3} \mathrm{PO}_{4}(\delta=0)$. - EI-MS $(70 \mathrm{eV})$ : $\mathrm{CH}$ 7-Gerät der Fa. Varian MAT oder 8222-Gerät der Fa. Finnigan MAT. Den angegebenen $m / z$-Werten für die Molekül-Ionen und die ausgewählten charakteristischen Fragmente liegen die Massenzahlen der jeweiligen Isotope mit der größten natürlichen relativen Häufigkeit zugrunde. - CI-MS $\left(\mathrm{NH}_{3}\right): 8430$-Gerät der Fa. Finnigan MAT.

(Acetoxymethyl)dimethylsilan (1 a), (Acetoxymethyl)methylphenylsilan (2a) und (Acetoxymethyl)diphenylsilan (3a) wurden gemäß Lit. $^{[1 \mathrm{~s}]}$ synthetisiert.

(Acetoxymethyl)methyl(1-naphthyl)silan (4a): Eine Mischung aus $4.00 \mathrm{~g}(19.8 \mathrm{mmol}) 25$ und $2.62 \mathrm{~g}(25.7 \mathrm{mmol})$ Essigsäureanhydrid wurde 70 min bei $70^{\circ} \mathrm{C}$ gerührt und das Produktgemisch sodann in Portionen von jeweils $1 \mathrm{~g}$ einer Kugelrohrdestillation unterworfen $\left(130^{\circ} \mathrm{C} / 0.001\right.$ Torr); Ausb. $4.45 \mathrm{~g}(92 \%)$ einer farblosen Flüssigkeit. - ${ }^{1} \mathrm{H}-\mathrm{NMR}\left(\mathrm{CDCl}_{3}\right): \delta=0.57\left(\mathrm{~d},{ }^{3} J_{\mathrm{HH}}=3.8 \mathrm{~Hz}, 3 \mathrm{H}\right.$ $\left.\mathrm{SiCH}_{3}\right), 1.99\left(\mathrm{~s}, 3 \mathrm{H} ; \mathrm{CCH}_{3}\right), 4.15\left(\delta_{\mathrm{A}}\right)$ und $4.22\left(\delta_{\mathrm{B}}\right)$ [AB-Teil des ABX-Systems $\mathrm{Si}\left(\mathrm{H}_{\mathrm{x}}\right) \mathrm{CH}_{\mathrm{A}} \mathrm{H}_{\mathrm{B}} \mathrm{O}, J_{\mathrm{AB}}=14.4 \mathrm{~Hz}, J_{\mathrm{AX}}=3.4 \mathrm{~Hz}$, $\left.J_{\mathrm{BX}}=2.6 \mathrm{~Hz}, 2 \mathrm{H} ; \mathrm{SiCH}_{2} \mathrm{O}\right], 4.93(\mathrm{~m}, 1 \mathrm{H} ; \mathrm{SiH}), 7.4-8.1(\mathrm{~m}, 7 \mathrm{H}$ $\left.\mathrm{SiC}_{10} \mathrm{H}_{7}\right) .-{ }^{13} \mathrm{C}-\mathrm{NMR}\left(\mathrm{CDCl}_{3}\right): \delta=-6.6\left(\mathrm{SiCH}_{3}\right), 20.7\left(\mathrm{CCH}_{3}\right)$, $55.3\left(\mathrm{SiCH}_{2} \mathrm{O}\right), 125.1,125.7,126.2,127.5,129.0,130.7,131.7,133.1$ 134.6 und $137.0\left(\mathrm{SiC}_{10} \mathrm{H}_{7}\right), 171.6(\mathrm{CO})$. - ${ }^{29} \mathrm{Si}-\mathrm{NMR}\left(\mathrm{C}_{6} \mathrm{D}_{6}\right): \delta=$ -19.7. - EI-MS: $m / z(\%)=244(5)\left[M^{+}\right], 243(13)\left[M^{+}-\mathrm{H}\right]$, $229(27)\left[M^{+}-\mathrm{CH}_{3}\right], 185(38)\left[M^{+}-\mathrm{OC}(\mathrm{O}) \mathrm{CH}_{3}\right], 171(41)$ $\left[M^{+}-\mathrm{CH}_{2} \mathrm{OC}(\mathrm{O}) \mathrm{CH}_{3}\right], 117(100)\left[M^{+}-\mathrm{C}_{10} \mathrm{H}_{7}\right]$.

$$
\mathrm{C}_{14} \mathrm{H}_{16} \mathrm{O}_{2} \mathrm{Si}(244.4) \text { Ber. C } 68.81 \text { H } 6.60 \text { Si } 11.49
$$
Gef. C 69.1 H 6.9 Si 11.5

Acetoxydimethyl(1-naphthyl)silan (4b): Darstellung durch Erhitzen von $4 \mathrm{a}\left(170^{\circ} \mathrm{C}, 33 \mathrm{~h}\right)$; farblose Flüssigkeit. - 'H-NMR $\left(\mathrm{CDCl}_{3}\right)$ : $\delta=0.73\left(\mathrm{~s}, 6 \mathrm{H} ; \mathrm{SiCH}_{3}\right), 2.10\left(\mathrm{~s}, 3 \mathrm{H} ; \mathrm{CCH}_{3}\right), 7.4-8.1(\mathrm{~m}, 7 \mathrm{H}$, $\left.\mathrm{SiC}_{10} \mathrm{H}_{7}\right) .-{ }^{13} \mathrm{C}-\mathrm{NMR}\left(\mathrm{CDCl}_{3}\right): \delta=-0.2\left(\mathrm{SiCH}_{3}\right), 22.9\left(\mathrm{CCH}_{3}\right)$,
$125.1,125.6,126.2,127.6,129.2,130.9,133.4,133.7,133.8$ und 136.3 $\left(\mathrm{SiC}_{10} \mathrm{H}_{7}\right), 171.4(\mathrm{CO}) .-$ EI-MS: $m / 2(\%)=244(19)\left[M^{+}\right], 229$ (93) $\left[M^{+}-\mathrm{CH}_{3}\right], 187(100)\left[\mathrm{C}_{11} \mathrm{H}_{11} \mathrm{OSi}^{+}\right]$.

$$
\mathrm{C}_{14} \mathrm{H}_{16} \mathrm{O}_{2} \mathrm{Si}(244.4) \text { Ber. C } 68.81 \text { H } 6.60 \text { Si } 11.49
$$

$$
\text { Gef. C } 68.7 \text { H } 6.8 \text { Si } 11.4
$$

(Acetoxymethyl) benzylmethylsilan (5a): Darstellung analog zur Synthese von $4 \mathrm{a}$ durch Umsetzung von $3.33 \mathrm{~g}(20.0 \mathrm{mmol}) 29 \mathrm{mit}$

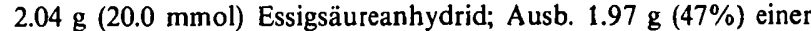
farblosen Flüssigkeit, Sdp. $58^{\circ} \mathrm{C} / 0.1$ Torr. - ${ }^{1} \mathrm{H}-\mathrm{NMR}\left(\mathrm{CDCl}_{3}\right)$ : $\delta=0.14\left(\mathrm{~d},{ }^{3} J_{\mathrm{HH}}=3.7 \mathrm{~Hz}, 3 \mathrm{H} ; \mathrm{SiCH}_{3}\right), 2.04\left(\mathrm{~s}, 3 \mathrm{H} ; \mathrm{CCH}_{3}\right), 2.27$ $\left(\mathrm{d},{ }^{3} \mathrm{~J}_{\mathrm{HH}}=3.2 \mathrm{~Hz}, 2 \mathrm{H} ; \mathrm{SiCH}_{2} \mathrm{C}\right), 3.83\left(\mathrm{~d},{ }^{3} \mathrm{~J}_{\mathrm{HH}}=2.6 \mathrm{~Hz} 2 \mathrm{H}\right.$ $\left.\mathrm{SiCH}_{2} \mathrm{O}\right), 4.03(\mathrm{~m}, 1 \mathrm{H} ; \mathrm{SiH}), 7.0-7.2\left(\mathrm{~m}, 5 \mathrm{H} ; \mathrm{CC}_{6} \mathrm{H}_{5}\right) .-{ }^{13} \mathrm{C}-\mathrm{NMR}$ $\left(\mathrm{CDCl}_{3}\right): \delta=-7.8\left(\mathrm{SiCH}_{3}\right), 20.7\left(\mathrm{CCH}_{3}\right), 21.1\left(\mathrm{SiCH}_{2} \mathrm{C}\right), 54.3$ $\left(\mathrm{SiCH}_{2} \mathrm{O}\right), 124.6,128.2,128.5$ und $138.6\left(\mathrm{CC}_{6} \mathrm{H}_{5}\right)$ - EI-MS: $\mathrm{m} / z=$ 208 (1) $\left[M^{+}\right], 207$ (1) $\left[M^{+}-\mathrm{H}\right], 149$ (4) $\left[M^{+}-\mathrm{OC}(\mathrm{O}) \mathrm{CH}_{3}\right]$ 135 (6) $\left[M^{+}-\mathrm{CH}_{2} \mathrm{OC}(\mathrm{O}) \mathrm{CH}_{3}\right], 117(100)\left[M^{+}-\mathrm{CH}_{2} \mathrm{C}_{6} \mathrm{H}_{5}\right]$.

$\mathrm{C}_{11} \mathrm{H}_{16} \mathrm{O}_{2} \mathrm{Si}$ (208.3) Ber. C $63.42 \mathrm{H} 7.74$ Si 13.48 Gef. C 62.9 H 7.5 Si 13.2

Acetoxybenzyldimethylsilan (5b): Darstellung durch Erhitzen von 5 a $\left(135^{\circ} \mathrm{C}, 65 \mathrm{~h}\right)$; farblose Flüssigkeit. $-{ }^{1} \mathrm{H}-\mathrm{NMR}\left(\mathrm{CDCl}_{3}\right)$ $\delta=0.26\left(\mathrm{~s}, 6 \mathrm{H} ; \mathrm{SiCH}_{3}\right), 2.04\left(\mathrm{~s}, 3 \mathrm{H} ; \mathrm{CCH}_{3}\right), 2.36\left(\mathrm{~s}, 2 \mathrm{H} ; \mathrm{SiCH}_{2} \mathrm{C}\right)$, 6.9-7.3 (m, 5 H; $\left.\mathrm{CC}_{6} \mathrm{H}_{5}\right) .-{ }^{13} \mathrm{C}-\mathrm{NMR}\left(\mathrm{CDCl}_{3}\right): \delta=-2.3\left(\mathrm{SiCH}_{3}\right)$, $22.8\left(\mathrm{CCH}_{3}\right), 25.8\left(\mathrm{SiCH}_{2} \mathrm{C}\right), 124.6,128.4(2 \mathrm{C})$ und $137.9\left(\mathrm{CC}_{6} \mathrm{H}_{5}\right)$ 172.0 (CO). - EI-MS: $m / z(\%)=208(6)\left[M^{+}\right], 193(22)\left[M^{+}-\right.$ $\left.\mathrm{CH}_{3}\right], 149(10)\left[M^{+}-\mathrm{OC}(\mathrm{O}) \mathrm{CH}_{3}\right], 117(100)\left[M^{+}-\mathrm{CH}_{2} \mathrm{C}_{6} \mathrm{H}_{5}\right]$ $\mathrm{C}_{11} \mathrm{H}_{16} \mathrm{O}_{2} \mathrm{Si}(208.3)$ Ber. C 63.42 H 7.74 Gef. C 63.2 H 7.8

(Acetoxymethyl)methyl/(trimethylsilyl)methyl]silan (6a): Darstellung analog zur Synthese von $4 \mathrm{a}$ durch Umsetzung von $3.25 \mathrm{~g}$ (20.0 mmol) $35 \mathrm{mit} 2.44 \mathrm{~g} \mathrm{(23.9} \mathrm{mmol)} \mathrm{Essigsäureanhydrid} \mathrm{(Reak-}$ tionszeit 3 h); Ausb. $2.83 \mathrm{~g} \mathrm{(69 \% )} \mathrm{einer} \mathrm{farblosen} \mathrm{Flüssigkeit,} \mathrm{Sdp}$ $32^{\circ} \mathrm{C} / 0.05$ Torr. $-{ }^{1} \mathrm{H}-\mathrm{NMR}\left(\mathrm{CDCl}_{3}\right): \delta=-0.25\left(\delta_{\mathrm{A}}\right)$ und -0.17 $\left(\delta_{\mathrm{B}}\right)$ [AB-Teil des ABX-Systems $\mathrm{Si}\left(\mathrm{H}_{\mathrm{X}}\right) \mathrm{CH}_{\mathrm{A}} \mathrm{H}_{\mathrm{B}} \mathrm{Si}, J_{\mathrm{AB}}=13.9 \mathrm{~Hz}$ $\left.J_{\mathrm{AX}}=4.9 \mathrm{~Hz}, J_{\mathrm{BX}}=3.2 \mathrm{~Hz}, 2 \mathrm{H} ; \mathrm{SiCH}_{2} \mathrm{Si}\right], 0.01\left[\mathrm{~s}, 9 \mathrm{H} ; \mathrm{Si}\left(\mathrm{CH}_{3}\right)_{3}\right]$, $0.12\left(\mathrm{~d},{ }^{3} J_{\mathrm{HH}}=3.7 \mathrm{~Hz}, 3 \mathrm{H} ; \mathrm{SiCH}_{3}\right), 2.01\left(\mathrm{~s}, 3 \mathrm{H} ; \mathrm{CCH}_{3}\right), 3.79(\mathrm{~d}$ $\left.{ }^{3} J_{\mathrm{HH}}=2.7 \mathrm{~Hz}, 2 \mathrm{H} ; \mathrm{SiCH}_{2} \mathrm{O}\right), 3.9-4.0(\mathrm{~m}, 1 \mathrm{H} ; \mathrm{SiH}) .-{ }^{13} \mathrm{C}-\mathrm{NMR}$ $\left(\mathrm{CDCl}_{3}\right): \delta=-5.0\left(\mathrm{SiCH}_{3}\right),-1.8\left(\mathrm{SiCH}_{2} \mathrm{Si}\right), 0.7\left[\mathrm{Si}\left(\mathrm{CH}_{3}\right)_{3}\right], 20.6$ $\left(\mathrm{CCH}_{3}\right), 56.5\left(\mathrm{SiCH}_{2} \mathrm{O}\right), 171.4(\mathrm{CO})$. - EI-MS: $m / z(\%)=204$ (3) $\left[M^{+}\right], 203(14)\left[M^{+}-\mathrm{H}\right], 189(20)\left[M^{+}-\mathrm{CH}_{3}\right], 145$ (12) $\left[M^{+}-\mathrm{OC}(\mathrm{O}) \mathrm{CH}_{3}\right], 131(100)\left[M^{+}-\mathrm{CH}_{2} \mathrm{OC}(\mathrm{O}) \mathrm{CH}_{3}\right], 117(64)$ $\left[M^{+}-\mathrm{CH}_{2} \mathrm{Si}\left(\mathrm{CH}_{3}\right)_{3}\right]$.

$$
\mathrm{C}_{8} \mathrm{H}_{20} \mathrm{O}_{2} \mathrm{Si}_{2} \text { (204.4) Ber. C } 47.01 \text { H } 9.86 \mathrm{Si} 27.48
$$

Gef. C 46.8 H 9.5 Si 27.0

Acetoxydimethyl/(trimethylsilyl)methyl/silan (6b): Darstellung durch Erhitzen von 6a $\left(135^{\circ} \mathrm{C}, 67 \mathrm{~h}\right)$; farblose Flüssigkeit. - ${ }^{1} \mathrm{H}$ $\operatorname{NMR}\left(\mathrm{CDCl}_{3}\right): \delta=0.02\left[\mathrm{~s}, 9 \mathrm{H} ; \mathrm{Si}\left(\mathrm{CH}_{3}\right)_{3}\right], 0.03\left(\mathrm{~s}, 2 \mathrm{H} ; \mathrm{SiCH}_{2} \mathrm{Si}\right)$ $0.26\left(\mathrm{~s}, 6 \mathrm{H} ; \mathrm{SiCH}_{3}\right), 2.01\left(\mathrm{~s}, 3 \mathrm{H} ; \mathrm{CCH}_{3}\right) .-{ }^{13} \mathrm{C}-\mathrm{NMR}\left(\mathrm{CDCl}_{3}\right): \delta=$ $0.9\left(\mathrm{SiCH}_{3}\right), 1.0\left[\mathrm{Si}\left(\mathrm{CH}_{3}\right)_{3}\right], 4.3\left(\mathrm{SiCH}_{2} \mathrm{Si}\right), 23.0\left(\mathrm{CCH}_{3}\right), 171.7(\mathrm{CO})$ - EI-MS: $m / z(\%)=204$ (1) $\left[M^{+}\right], 189(34)\left[M^{+}-\mathrm{CH}_{3}\right], 145$ (5) $\left[M^{+}-\mathrm{OC}(\mathrm{O}) \mathrm{CH}_{3}\right], 131$ (86) $\left[M^{+}-\mathrm{Si}\left(\mathrm{CH}_{3}\right)_{3}\right], 73(100)$ $\left[\mathrm{Si}\left(\mathrm{CH}_{3}\right)_{3}^{+}\right]$

$\mathrm{C}_{8} \mathrm{H}_{20} \mathrm{O}_{2} \mathrm{Si}_{2}$ (204.4) Ber. C 47.01 H 9.86 Gef. C 46.9 H 9.9

(Acetoxymethyl)bis ( trimethylsilyl)methyl]silan (7a): Darstellung analog zur Synthese von 4a durch Umsetzung von $3.24 \mathrm{~g}(13.8$ $\mathrm{mmol}) 36 \mathrm{mit} 1.55 \mathrm{~g}(15.2 \mathrm{mmol})$ Essigsäureanhydrid (Reaktionszeit $1.5 \mathrm{~h})$; Ausb. $2.55 \mathrm{~g}(67 \%)$ einer farblosen Flüssigkeit, Sdp. $67^{\circ} \mathrm{C} /$ 0.1 Torr. - ${ }^{1} \mathrm{H}-\mathrm{NMR}\left(\mathrm{CDCl}_{3}\right): \delta=-0.22\left(\delta_{\mathrm{A}}\right)$ und $-0.16\left(\delta_{\mathrm{B}}\right)$ [AB-Teil des ABX-Systems $\mathrm{Si}\left(\mathrm{H}_{\mathrm{X}}\right) \mathrm{CH}_{\mathrm{A}} \mathrm{H}_{\mathrm{B}} \mathrm{Si}, J_{\mathrm{AB}}=13.9 \mathrm{~Hz}, J_{\mathrm{AX}}=$ $\left.3.5 \mathrm{~Hz}, J_{\mathrm{BX}}=4.3 \mathrm{~Hz}, 4 \mathrm{H} ; \mathrm{SiCH}_{2} \mathrm{Si}\right], 0.02\left(\mathrm{~s}, 18 \mathrm{H} ; \mathrm{SiCH}_{3}\right), 2.02(\mathrm{~s}$ $\left.3 \mathrm{H} ; \mathrm{CCH}_{3}\right), 3.78\left(\mathrm{~d},{ }^{3} \mathrm{~J}_{\mathrm{HH}}=2.5 \mathrm{~Hz}, 2 \mathrm{H} ; \mathrm{SiCH}_{2} \mathrm{O}\right), 4.02(\mathrm{~m}, 1 \mathrm{H}$ $\mathrm{SiH}) .-{ }^{13} \mathrm{C}-\mathrm{NMR}\left(\mathrm{CDCl}_{3}\right): \delta=-0.7\left(\mathrm{SiCH}_{2} \mathrm{Si}\right), 0.8\left[\mathrm{Si}\left(\mathrm{CH}_{3}\right)_{3}\right]$, $20.8\left(\mathrm{CCH}_{3}\right), 56.8\left(\mathrm{SiCH}_{2} \mathrm{O}\right), 171.5(\mathrm{CO}) .-\mathrm{EI}-\mathrm{MS}: \mathrm{m} / z(\%)=276$ 
(7) $\left[M^{+}\right], 275(26)\left[M^{+}-\mathrm{H}\right], 233(48)\left[M^{+}-\mathrm{C}(\mathrm{O}) \mathrm{CH}_{3}\right], 203$ (90) $\left[M^{+}-\mathrm{CH}_{2} \mathrm{OC}(\mathrm{O}) \mathrm{CH}_{3}\right], 189(96)\left[M^{+}-\mathrm{CH}_{2} \mathrm{Si}\left(\mathrm{CH}_{3}\right)_{3}\right], 73$ (100) $\left[\mathrm{Si}\left(\mathrm{CH}_{3}\right)_{3}^{+}\right]$.

$$
\mathrm{C}_{11} \mathrm{H}_{28} \mathrm{O}_{2} \mathrm{Si}_{3} \text { (276.6) Ber. C } 47.77 \text { H } 10.20 \text { Si } 30.46
$$
Gef. C 47.8 H 10.5 Si 30.5

Acetoxymethylbis/(trimethylsilyl)methyl]silan (7b): Darstellung durch Erhitzen von 7 a $\left(135^{\circ} \mathrm{C}, 60 \mathrm{~h}\right)$; farblose Flüssigkeit. - ' $\mathrm{H}$ NMR $\left(\mathrm{CDCl}_{3}\right)$ : $\delta=-0.01\left(\delta_{\mathrm{A}}\right)$ und $0.13\left(\delta_{\mathrm{B}}\right)\left(\mathrm{AB}-\mathrm{System}, J_{\mathrm{AB}}=\right.$ $\left.13.8 \mathrm{~Hz}, 4 \mathrm{H} ; \mathrm{SiCH}_{2} \mathrm{Si}\right), 0.04\left[\mathrm{~s}, 18 \mathrm{H} ; \mathrm{Si}\left(\mathrm{CH}_{3}\right)_{3}\right], 0.29\left(\mathrm{~s}, 3 \mathrm{H} ; \mathrm{SiCH}_{3}\right)$, $2.02\left(\mathrm{CCH}_{3}\right) .-{ }^{13} \mathrm{C}-\mathrm{NMR}\left(\mathrm{CDCl}_{3}\right): \delta=1.2\left[\mathrm{Si}\left(\mathrm{CH}_{3}\right)_{3}\right], 1.9\left(\mathrm{SiCH}_{3}\right)$, $5.5\left(\mathrm{SiCH}_{2} \mathrm{Si}\right), 23.2\left(\mathrm{CCH}_{3}\right), 171.6(\mathrm{CO})$ - EI-MS: $m / z(\%)=261$ (100) $\left[M^{+}-\mathrm{CH}_{3}\right], 217(6)\left[M^{+}-\mathrm{OC}(\mathrm{O}) \mathrm{CH}_{3}\right], 203(98)\left[M^{+}-\right.$ $\left.\mathrm{Si}\left(\mathrm{CH}_{3}\right)_{3}\right]$.

$$
\begin{array}{lllll}
\mathrm{C}_{11} \mathrm{H}_{28} \mathrm{O}_{2} \mathrm{Si}_{3} \text { (276.6) } & \text { Ber. C } 47.77 \text { H } 10.20 \text { Si } 30.46 \\
& \text { Gef. C } 47.5 \text { H } 10.2 \text { Si } 30.3
\end{array}
$$

(Benzoyloxymethyl)methyl(1-naphthyl)silan (8a): Zu einer Lösung von $5.00 \mathrm{~g}(24.7 \mathrm{mmol}) 25$ und $2.50 \mathrm{~g}(24.7 \mathrm{mmol})$ Triethylamin in $200 \mathrm{ml}$ Petrolether wurde bei $-70^{\circ} \mathrm{C}$ unter Rühren innerhalb von 20 min eine Lösung von $3.47 \mathrm{~g}(24.7 \mathrm{mmol})$ Benzoylchlorid in $25 \mathrm{ml}$ Petrolether getropft. Nach beendeter Zugabe rührte man $20 \mathrm{~h}$ bei Raumtemp., filtrierte und befreite das Filtrat unter vermindertem Druck vom Lösungsmittel. Der Rückstand wurde in $40 \mathrm{ml}$ Diethylether aufgenommen und die etherische Lösung mit $20 \mathrm{ml}$ Wasser gewaschen. Man extrahierte die wäßrige Phase zweimal mit je $20 \mathrm{ml}$ Diethylether, trocknete die vereinigten organischen Extrakte mit $\mathrm{Na}_{2} \mathrm{SO}_{4}$, entfernte das Lösungsmittel unter vcrmindertem Druck und kristallisierte den Rückstand bei $-20^{\circ} \mathrm{C}$ aus Diethylether/Petrolether (1/2, v/v); Ausb. $6.12 \mathrm{~g}(81 \%)$ einer bei Raumtemp. flüssigen, farblosen Substanz. - 'H-NMR (CDCl $)_{3}$ : $\delta=0.65\left(\mathrm{~d},{ }^{3} J_{\mathrm{HH}}=3.8 \mathrm{~Hz}, 3 \mathrm{H} ; \mathrm{SiCH}_{3}\right), 4.40\left(\delta_{\mathrm{A}}\right)$ und $4.46\left(\delta_{\mathrm{B}}\right)$ [AB-Teil des ABX-Systems $\mathrm{Si}\left(\mathrm{H}_{\mathrm{X}}\right) \mathrm{CH}_{\mathrm{A}} \mathrm{H}_{\mathrm{B}} \mathrm{O}, J_{\mathrm{AB}}=14.35 \mathrm{~Hz}$, $\left.J_{\mathrm{AX}}=3.2 \mathrm{~Hz}, J_{\mathrm{BX}}=2.6 \mathrm{~Hz}, 2 \mathrm{H} ; \mathrm{SiCH}_{2} \mathrm{O}\right], 5.04(\mathrm{~m}, 1 \mathrm{H} ; \mathrm{SiH})$, 7.3-8.2 (m, $\left.12 \mathrm{H} ; \mathrm{SiC}_{10} \mathrm{H}_{7}, \mathrm{CC}_{6} \mathrm{H}_{5}\right) .-{ }^{13} \mathrm{C}-\mathrm{NMR}\left(\mathrm{CDCl}_{3}\right): \delta=$ -6.5 $\left(\mathrm{SiCH}_{3}\right), 55.7\left(\mathrm{SiCH}_{2} \mathrm{O}\right), 125.2,125.8,126.4,127.6,128.3,129.1$, 129.6, 130.3, 130.8, 131.8, 132.8, 134.7 und $137.1\left(\mathrm{SiC}_{10} \mathrm{H}_{7}, \mathrm{CC}_{6} \mathrm{H}_{5}\right)$, 167.3 (CO). - EI-MS: $m / z(\%)=305(4)\left[M^{+}-\mathrm{H}\right], 179(44)$ $\left[M^{+}-\mathrm{C}_{10} \mathrm{H}_{7}\right], 105(100)\left[\mathrm{C}_{6} \mathrm{H}_{5} \mathrm{CO}^{+}\right]$.

\section{$\mathrm{C}_{19} \mathrm{H}_{18} \mathrm{O}_{2} \mathrm{Si}$ (306.4) Ber. C 74.47 H 5.92 Gef. C 74.9 H 5.9}

(Benzoyloxy)dimethyl(1-naphthyl)silan (8b): Darstellung durch Erhitzen von 8a $\left(150^{\circ} \mathrm{C}, 48 \mathrm{~h}\right)$; farblose Flüssigkeit. - ${ }^{1} \mathrm{H}-\mathrm{NMR}$ $\left(\mathrm{C}_{6} \mathrm{D}_{6}\right): \delta=0.78\left(\mathrm{~s}, 6 \mathrm{H} ; \mathrm{SiCH}_{3}\right), 7.0-8.3\left(\mathrm{~m}, 12 \mathrm{H} ; \mathrm{SiC}_{10} \mathrm{H}_{7}, \mathrm{CC}_{6} \mathrm{H}_{5}\right)$. - ${ }^{13} \mathrm{C}$-NMR $\left(\mathrm{C}_{6} \mathrm{D}_{6}\right): \delta=0.7\left(\mathrm{SiCH}_{3}\right), 126.0,126.6,127.2,128.8$, $129.2,130.2,131.3,132.0,132.5,133.7,134.7,134.8,135.1$ und 137.7 $\left(\mathrm{SiC}_{10} \mathrm{H}_{7}, \mathrm{CC}_{6} \mathrm{H}_{5}\right), 167.1(\mathrm{CO}) .-$ EI-MS: $m / z(\%)=306(6)\left[M^{+}\right]$, $291(100)\left[M^{+}-\mathrm{CH}_{3}\right], 179(26)\left[M^{+}-\mathrm{C}_{10} \mathrm{H}_{7}\right]$.

\section{$\mathrm{C}_{19} \mathrm{H}_{18} \mathrm{O}_{2} \mathrm{Si}$ (306.4) Ber. C 74.47 H 5.92 Gef. C 74.9 H 6.1}

(Benzoyloxymethyl)diphenylsilan (9a): Darstellung analog zur Synthese von $8 \mathrm{a}$ durch Umsetzung von $5.00 \mathrm{~g}(23.3 \mathrm{mmol}) 37 \mathrm{mit}$ $2.53 \mathrm{~g}(25.0 \mathrm{mmol})$ Triethylamin und $3.51 \mathrm{~g}(25.0 \mathrm{mmol})$ Benzoylchlorid in $200 \mathrm{ml}$ Pentan; Ausb. $6.77 \mathrm{~g}(91 \%)$ weiße Kristalle, Schmp. $38^{\circ} \mathrm{C}$ (Aceton). $-{ }^{1} \mathrm{H}-\mathrm{NMR}\left(\mathrm{CDCl}_{3}\right): \delta=4.56\left(\mathrm{~d},{ }^{3} J_{\mathrm{HH}}=\right.$ $\left.2.9 \mathrm{~Hz}, 2 \mathrm{H} ; \mathrm{SiCH}_{2} \mathrm{O}\right), 5.13\left(\mathrm{t},{ }^{3} \mathrm{JHH}_{\mathrm{HH}}=2.9 \mathrm{~Hz}, 1 \mathrm{H} ; \mathrm{SiH}\right), 7.4-8.0(\mathrm{~m}$ $\left.15 \mathrm{H} ; \mathrm{SiC}_{6} \mathrm{H}_{5}, \mathrm{CC}_{6} \mathrm{H}_{5}\right) .-{ }^{13} \mathrm{C}-\mathrm{NMR}\left(\mathrm{CDCl}_{3}\right): \delta=54.4\left(\mathrm{SiCH}_{2} \mathrm{O}\right)$, $128.2,128.3,129.3,130.2,130.3,131.4,132.8$ und $135.4\left(\mathrm{SiC}_{6} \mathrm{H}_{5}\right.$ $\left.\mathrm{CC}_{6} \mathrm{H}_{5}\right), 167.2(\mathrm{CO}) .-$ EI-MS: $m / z(\%)=318(6)\left[M^{+}\right], 317(20)$ $\left[M^{+}-\mathrm{H}\right], 241(100)\left[M^{+}-\mathrm{C}_{6} \mathrm{H}_{5}\right]$.

$\mathrm{C}_{20} \mathrm{H}_{18} \mathrm{O}_{2} \mathrm{Si} \mathrm{(318.4)}$ Ber. C 75.43 H 5.70 Gef. C 76.0 H 5.8

(Benzoyloxy)methyldiphenylsilan (9b): Darstellung durch Erhitzen von $9 \mathrm{a}\left(150^{\circ} \mathrm{C}, 24 \mathrm{~h}\right)$; farblose Flüssigkeit. - ${ }^{1} \mathrm{H}-\mathrm{NMR}\left(\mathrm{C}_{6} \mathrm{D}_{6}\right)$ : $\delta=0.93\left(\mathrm{~s}, 3 \mathrm{H} ; \mathrm{SiCH}_{3}\right), 7.0-8.2\left(\mathrm{~m}, 15 \mathrm{H} ; \mathrm{SiC}_{6} \mathrm{H}_{5}, \mathrm{CC}_{6} \mathrm{H}_{5}\right) .{ }^{13} \mathrm{C}-$
$\operatorname{NMR}\left(\mathrm{C}_{6} \mathrm{D}_{6}\right): \delta=-2.3\left(\mathrm{SiCH}_{3}\right), 127.9,128.5,130.5,130.6,131.8$, 133.1, 134.5 und $135.0\left(\mathrm{SiC}_{6} \mathrm{H}_{5}, \mathrm{CC}_{6} \mathrm{H}_{5}\right), 166.3(\mathrm{CO})$. - EI-MS: $m / z(\%)=303(10)\left[M^{+}-\mathrm{CH}_{3}\right], 241(42)\left[M^{+}-\mathrm{C}_{6} \mathrm{H}_{5}\right], 197$ (100) $\left[M^{+}-\mathrm{OC}(\mathrm{O}) \mathrm{C}_{6} \mathrm{H}_{5}\right]$.

\section{$\mathrm{C}_{20} \mathrm{H}_{18} \mathrm{O}_{2} \mathrm{Si} \mathrm{(318.4)}$ Ber. C 75.43 H 5.70 Gef. C 75.4 H 6.1}

Diphenyl(trifluoracetoxymethyl) silan (10a): $\mathrm{Zu}$ einer Lösung von $2.14 \mathrm{~g}(9.98 \mathrm{mmol}) 37$ und $1.01 \mathrm{~g}(9.98 \mathrm{mmol})$ Triethylamin in $30 \mathrm{ml}$ Diethylether $/$ Pentan $(1 / 2, v / v)$ wurde bei $-40^{\circ} \mathrm{C}$ unter Rühren innerhalb von 5 min einc Lösung von $2.10 \mathrm{~g}(10.0 \mathrm{mmol})$ Trifluoressigsäureanhydrid in $20 \mathrm{ml}$ Pentan getropft. Nach beendeter Zugabe rührte man weitere $10 \mathrm{~min}$ bei $-40^{\circ} \mathrm{C}$, extrahierte dann zügig dreimal mit je $10 \mathrm{ml}$ Wasser, trocknete die etherische Phase mit $\mathrm{Na}_{2} \mathrm{SO}_{4}$, befreite unter vermindertem Druck vom Lösungsmittel und kristallisierte den Rückstand bej $-20^{\circ} \mathrm{C}$ aus Pentan; Ausb. $2.94 \mathrm{~g}(95 \%)$, weiße Kristalle, Schmp. ca. $17^{\circ} \mathrm{C}$ - ${ }^{1} \mathrm{H}-\mathrm{NMR}$ $\left(\mathrm{CDCl}_{3}\right): \delta=4.57\left(\mathrm{~d},{ }^{3} \mathrm{~J}_{\mathrm{HH}}=2.9 \mathrm{~Hz}, 2 \mathrm{H} ; \mathrm{SiCH}_{2} \mathrm{O}\right), 5.08\left(\mathrm{t},{ }^{3} \mathrm{~J}_{\mathrm{HH}}=\right.$ $2.9 \mathrm{~Hz}, 1 \mathrm{H} ; \mathrm{SiH}), 7.3-7.6\left(\mathrm{~m}, 10 \mathrm{H} ; \mathrm{SiC}_{6} \mathrm{H}_{5}\right) .-{ }^{13} \mathrm{C}-\mathrm{NMR}\left(\mathrm{CDCl}_{3}\right)$ : $\delta=58.2\left(\mathrm{SiCH}_{2} \mathrm{O}\right), 114.6\left(\mathrm{q},{ }^{1} J_{\mathrm{CF}}=285.1 \mathrm{~Hz} ; \mathrm{CCF}_{3}\right), 128.4,129.8$, 130.7 und $135.4\left(\mathrm{SiC}_{6} \mathrm{H}_{5}\right), 158.0\left(\mathrm{q},{ }^{2} J_{\mathrm{CF}}=42.2 \mathrm{~Hz} ; \mathrm{CO}\right) .-{ }^{29} \mathrm{Si}$ NMR $\left(\mathrm{CDCl}_{3}\right): \delta=-21.7$. - EI-MS: $m / 2(\%)=309(16)\left[M^{+}\right.$ $-\mathrm{H}], 233(36)\left[M^{+}-\mathrm{C}_{6} \mathrm{H}_{5}\right], 183(100)\left[M^{+}-\mathrm{CH}_{2} \mathrm{OC}(\mathrm{O}) \mathrm{CF}_{3}\right]$. $\mathrm{C}_{15} \mathrm{H}_{13} \mathrm{~F}_{3} \mathrm{O}_{2} \mathrm{Si}$ (310.3) Ber. C 58.05 H 4.22 Gef. C 58.3 H 4.2

Methyldiphenyl(trifluoracetoxy)silan (10b): Darstellung durch Erhitzen von $10 \mathrm{a}\left(150^{\circ} \mathrm{C}, 12 \mathrm{~h}\right)$; farblose Flüssigkeit. - ' $\mathrm{H}$-NMR $\left(\mathrm{CDCl}_{3}\right): \delta=0.98\left(\mathrm{~s}, 3 \mathrm{H} ; \mathrm{SiCH}_{3}\right), 7.4-7.7\left(\mathrm{~m}, 10 \mathrm{H} ; \mathrm{SiC}_{6} \mathrm{H}_{5}\right)$. ${ }^{13} \mathrm{C}-\mathrm{NMR}\left(\mathrm{CDCl}_{3}\right): \delta=-2.5\left(\mathrm{SiCH}_{3}\right), 114.6\left(\mathrm{q},{ }^{\prime} J_{\mathrm{CF}}=286.0 \mathrm{~Hz}\right.$; $\left.\mathrm{CCF}_{3}\right), 128.9,131.8,132.0$ und $135.0\left(\mathrm{SiC}_{6} \mathrm{H}_{5}\right), 156.4\left(\mathrm{q},{ }^{2} J_{\mathrm{CF}}=\right.$ $42.6 \mathrm{~Hz} ; \mathrm{CO})$. - EI-MS: $m / z(\%)=310(12)\left[M^{+}\right], 295(11)\left[M^{+}\right.$ $\left.-\mathrm{CH}_{3}\right], 201$ (100), $105(30)\left[\mathrm{SiC}_{6} \mathrm{H}_{5}^{+}\right]$.

$\mathrm{C}_{15} \mathrm{H}_{13} \mathrm{~F}_{3} \mathrm{O}_{2} \mathrm{Si}$ (310.3) Ber. C 58.05 H 4.22 Gef. C 58.3 H 4.5

Dimethyl(trifluormethylsulfonyloxymethyl)silan (11a): Darstellung gemäß Lit. ${ }^{[t c]}$.

Deuteriodimethyl(trifluormethylsulfonyloxymethyl)silan ([D]11a): Darstellung in Analogie zu 11 a durch Umsetzung von [D]38 mit Trifluormethansulfonsäureanhydrid; Ausb. $71 \%$. - ' $\mathrm{H}$ $\operatorname{NMR}\left(\mathrm{CDCl}_{3}\right): \delta=0.24\left(1: 1: 1-\mathrm{t},{ }^{3} J_{\mathrm{HD}}=0.5 \mathrm{~Hz}, 6 \mathrm{H} ; \mathrm{SiCH}_{3}\right), 4.35$ (,s", $\left.2 \mathrm{H} ; \mathrm{SiCH}_{2} \mathrm{O}\right) .-{ }^{13} \mathrm{C}-\mathrm{NMR}\left(\mathrm{CDCl}_{3}\right): \delta=-6.9\left(\mathrm{SiCH}_{3}\right), 69.5$ $\left(\mathrm{SiCH}_{2} \mathrm{O}\right), 118.9\left(\mathrm{q},{ }^{1} J_{\mathrm{CF}}=320.8 \mathrm{~Hz} ; \mathrm{SCF}_{3}\right) .-{ }^{29} \mathrm{Si}-\mathrm{NMR}\left(\mathrm{C}_{6} \mathrm{D}_{6}\right)$ : $\delta=-15.9\left(1: 1: 1-\mathrm{t},{ }^{1} J_{\text {SiD }}=30.4 \mathrm{~Hz}\right)$.

\section{$\mathrm{C}_{4} \mathrm{H}_{8} \mathrm{DF}_{3} \mathrm{O}_{3} \mathrm{SSi}(223.3) \quad$ Ber. C $21.52 \mathrm{H}(\mathrm{D}) 4.51 \mathrm{~S} 14.36$ Gef. C $21.8 \quad H(D) 4.3 \quad$ S 14.5}

Methylphenyl (trifluormethylsulfonyloxymethyl) silan (12a): Darstellung gemäß Lit. ${ }^{[1 c]}$.

Diphenyl(trifluormethylsulfonyloxymethyl)silan (13a): Zu einer Lösung von $6.50 \mathrm{~g}(30.3 \mathrm{mmol}) 37$ und $3.40 \mathrm{~g}(33.6 \mathrm{mmol})$ Triethylamin in $35 \mathrm{ml}$ Tetrachlormethan wurde bei $-60^{\circ} \mathrm{C}$ unter Rühren innerhalb von $5 \mathrm{~min}$ eine Lösung von $9.20 \mathrm{~g}$ (32.6 mmol) Trifluormethansulfonsäureanhydrid in $35 \mathrm{ml}$ Tetrachlormethan getropft. Nach Entfernen des Kühlbads rührte man weitere $5 \mathrm{~min}$, trennte durch Dekantieren von dem ölig anfallenden Triethylammoniumtrifluormethansulfonat $a b$, wusch die Tetrachlormethan-Phase zweimal mit je $30 \mathrm{ml}$ Wasser, trocknete mit $\mathrm{Na}_{2} \mathrm{SO}_{4}$ und befreite unter vermindertem Druck vom Lösungsmittel. Der Rückstand wurde in $10 \mathrm{ml}$ Pentan aufgenommen und bei $-60^{\circ} \mathrm{C}$ kristallisiert; Ausb. $5.2 \mathrm{~g} \mathrm{(50 \% )}$ einer bei Raumtemp. farblosen Flüssigkeit. ${ }^{1} \mathrm{H}-\mathrm{NMR}\left(\mathrm{CDCl}_{3}\right): \delta=4.72\left(\mathrm{qd},{ }^{3} J_{\mathrm{HH}}=2.8 \mathrm{~Hz},{ }^{5} J_{\mathrm{HF}}=0.3 \mathrm{~Hz}\right.$, $\left.2 \mathrm{H} ; \mathrm{SiCH}_{2} \mathrm{O}\right), 5.10\left(\mathrm{t},{ }^{3} \mathrm{~J}_{\mathrm{HH}}=2.8 \mathrm{~Hz}, 1 \mathrm{H} ; \mathrm{SiH}\right), 7.3-7.6(\mathrm{~m}, 10 \mathrm{H}$; $\left.\mathrm{SiC}_{6} \mathrm{H}_{5}\right) .-{ }^{13} \mathrm{C}-\mathrm{NMR}\left(\mathrm{CDCl}_{3}\right): \delta=67.4\left(\mathrm{SiCH}_{2} \mathrm{O}\right), 118.8\left(\mathrm{q},{ }^{1} J_{\mathrm{CF}}=\right.$ $\left.320.4 \mathrm{~Hz} ; \mathrm{SCF}_{3}\right), 128.6,128.8,131.1$ und $135.4\left(\mathrm{SiC}_{6} \mathrm{H}_{5}\right) .-{ }^{29} \mathrm{Si}-$ NMR $\left(\mathrm{CDCl}_{3}\right): \delta=-21.6$. - EI-MS: $m / z(\%)=346(5)\left[M^{+}\right]$, 
$345(12)\left[M^{+}-\mathrm{H}\right], 269(18)\left[M^{+}-\mathrm{C}_{6} \mathrm{H}_{5}\right], 197(18)\left[M^{+}-\right.$ $\left.\mathrm{OS}(\mathrm{O})_{2} \mathrm{CF}_{3}\right], 183(100)\left[M^{+}-\mathrm{CH}_{2} \mathrm{OS}(\mathrm{O})_{2} \mathrm{CF}_{3}\right]$.

$\mathrm{C}_{14} \mathrm{H}_{13} \mathrm{~F}_{3} \mathrm{O}_{3} \mathrm{SSi}$ (346.4) Ber. C 48.54 H 3.78 Gef. C 48.7 H 3.6

Methyldiphenyl(trifluormethylsulfonyloxy) silan (13b): Darstellung durch Erhitzen von $13 \mathrm{a}\left(110^{\circ} \mathrm{C}, 1.5 \mathrm{~h}\right)$; farblose Flüssigkeit. - 'H-NMR $\left(\mathrm{C}_{6} \mathrm{D}_{6}\right): \delta=0.68\left(\mathrm{~s}, 3 \mathrm{H} ; \mathrm{SiCH}_{3}\right), 7.0-7.2$ und 7.4-7.5 $\left(\mathrm{m}, 10 \mathrm{H} ; \mathrm{SiC}_{6} \mathrm{H}_{5}\right) .-{ }^{13} \mathrm{C}-\mathrm{NMR}\left(\mathrm{C}_{6} \mathrm{D}_{6}\right): \delta=2.7\left(\mathrm{SiCH}_{3}\right), 121.9(\mathrm{q}$, $\left.{ }^{1} J_{\mathrm{CF}}=317.8 \mathrm{~Hz} ; \mathrm{SCF}_{3}\right), 129.3,131.2,132.5$ und $135.3\left(\mathrm{SiC}_{6} \mathrm{H}_{5}\right)$. EI-MS: $m / z(\%)=346(40)\left[M^{+}\right], 331(38)\left[M^{+}-\mathrm{CH}_{3}\right], 277(3)$ $\left[M^{+}-\mathrm{CF}_{3}\right], 201(100), 197(36)\left[M^{+}-\mathrm{OS}(\mathrm{O})_{2} \mathrm{CF}_{3}\right] .-$ MSHochauflösung für $\mathrm{C}_{14} \mathrm{H}_{13} \mathrm{~F}_{3} \mathrm{O}_{3} \mathrm{SSi}\left[M^{+}\right]$: 346.0307 (ber.), 346.0286 (gef.); MS-Hochauflösung für $\left[M^{+}-\mathrm{CH}_{3}\right]: 331.0072$ (ber.), 331.0079 (gef.)

(Diphenylphosphinyloxymethyl) dimethylsilan (14a): Zu einer Lösung von $4.88 \mathrm{~g}(54.1 \mathrm{mmol}) 38$ und $5.60 \mathrm{~g}(55.3 \mathrm{mmol})$ Triethylamin in $40 \mathrm{ml}$ Toluol wurde bei $0^{\circ} \mathrm{C}$ unter Rühren innerhalb von $10 \mathrm{~min}$ eine Lösung von $11.7 \mathrm{~g}(49.4 \mathrm{mmol})$ Diphenylphosphinsäurechlorid in $40 \mathrm{ml}$ Toluol getropft. Man rührte $1 \mathrm{~h}$ bei $0^{\circ} \mathrm{C}$ und $15 \mathrm{~h}$ bei Raumtemp. und versetzte dann mit $40 \mathrm{ml} 1 \mathrm{~m}$ Salzsäure und $100 \mathrm{ml}$ Diethylether. Die organische Phase wurde abgetrennt und nacheinander mit $20 \mathrm{ml}$ Wasser, $40 \mathrm{ml} 5$ proz. wäßriger $\mathrm{NaHCO}_{3}$-Lösung und $20 \mathrm{ml}$ Wasser gewaschen. Dann trocknete man mit $\mathrm{Na}_{2} \mathrm{SO}_{4}$, filtrierte durch eine $5 \mathrm{~cm}$ dicke Schicht von Kieselgel 60 (aufgeschwemmt in Diethylether), befreite unter vermindertem Druck vom Lösungsmittel und kristallisierte den Rückstand bei $-20^{\circ} \mathrm{C}$ aus Petrolether; Ausb. $10.0 \mathrm{~g}(70 \%)$ weiße Kristalle, Schmp. $38-39^{\circ} \mathrm{C} .-{ }^{1} \mathrm{H}-\mathrm{NMR}\left(\mathrm{C}_{6} \mathrm{D}_{6}\right): \delta=-0.02\left(\mathrm{~d},{ }^{3} \mathrm{~J}_{\mathrm{HH}}=3.6 \mathrm{~Hz}, 6 \mathrm{H}\right.$; $\left.\mathrm{SiCH}_{3}\right), 3.72\left(\mathrm{dd},{ }^{3} J_{\mathrm{HH}}=2.6 \mathrm{~Hz},{ }^{3} J_{\mathrm{HP}}=6.9 \mathrm{~Hz}, 2 \mathrm{H} ; \mathrm{SiCH}_{2} \mathrm{O}\right), 4.08$ (tsept, ${ }^{3} J_{\mathrm{HH}}=2.6 \mathrm{~Hz},{ }^{3} J_{\mathrm{HH}}=3.6 \mathrm{~Hz}, 1 \mathrm{H} ; \mathrm{SiH}$ ), $7.0-7.1$ und $7.8-7.9\left(\mathrm{~m}, 10 \mathrm{H} ; \mathrm{PC}_{6} \mathrm{H}_{5}\right) .-{ }^{13} \mathrm{C}-\mathrm{NMR}\left(\mathrm{CDCl}_{3}\right): \delta=-6.3\left(\mathrm{SiCH}_{3}\right)$, $55.5\left(\mathrm{~d},{ }^{2} J_{\mathrm{CP}}=8.3 \mathrm{~Hz}, 2 \mathrm{H} ; \mathrm{SiCH}_{2} \mathrm{O}\right), 128.7\left(\mathrm{~d}, J_{\mathrm{CP}}=12.8 \mathrm{~Hz}\right), 131.6$ (d, $\left.J_{\mathrm{CP}}=2.8 \mathrm{~Hz}\right), 132.1\left(\mathrm{~d}, J_{\mathrm{CP}}=9.6 \mathrm{~Hz}\right)$ und $133.0\left(\mathrm{~d}, J_{\mathrm{CP}}=\right.$ $135.1 \mathrm{~Hz})\left(\mathrm{PC}_{6} \mathrm{H}_{5}\right)$. - ${ }^{31} \mathrm{P}-\mathrm{NMR}\left(\mathrm{C}_{6} \mathrm{D}_{6}\right): \delta=31.6 .-{ }^{29} \mathrm{Si}-\mathrm{NMR}$ $\left(\mathrm{C}_{6} \mathrm{D}_{6}\right): \delta=-14.9\left(\mathrm{~d},{ }^{3} J_{\mathrm{SiP}}=10.8 \mathrm{~Hz}\right) .-\mathrm{EI}-\mathrm{MS}: m / z(\%)=289$ (100) $\left[M^{+}-\mathrm{H}\right], 275(40)\left[M^{+}-\mathrm{CH}_{3}\right]$.

$\mathrm{C}_{15} \mathrm{H}_{19} \mathrm{O}_{2}$ PSi (290.4) Ber. C 62.05 H 6.59 Si 9.67 Gef. C $61.8 \quad$ H $6.7 \quad$ Si 9.4

(Diphenylphosphinyloxy)trimethylsilan (14b): Darstellung durch Erhitzen einer $0.4 \mathrm{M}$ Lösung von $14 \mathrm{a}$ in Toluol $\left(100^{\circ} \mathrm{C}, 12 \mathrm{~h}\right)$ und nachfolgende Verdampfung des Lösungsmittels im Vakuum; weiBer, nicht ganz einheitlicher Feststoff. - ${ }^{3} \mathrm{H}-\mathrm{NMR}\left(\mathrm{C}_{6} \mathrm{D}_{6}\right): \delta=0.18$ (s, 9H; $\left.\mathrm{SiCH}_{3}\right), 7.05-7.1$ und $7.8-7.9\left(\mathrm{~m}, 10 \mathrm{H} ; \mathrm{PC}_{6} \mathrm{H}_{5}\right) .-{ }^{13} \mathrm{C}-$ NMR $\left(\mathrm{C}_{6} \mathrm{D}_{6}\right): \delta=1.3\left(\mathrm{SiCH}_{3}\right), 128.5\left(\mathrm{~d}, J_{\mathrm{CP}}=13.0 \mathrm{~Hz}\right), 131.5(\mathrm{~d}$, $\left.J_{\mathrm{CP}}=10.1 \mathrm{~Hz}\right), 131.6\left(\mathrm{~d}, J_{\mathrm{CP}}=2.8 \mathrm{~Hz}\right)$ und $135.7\left(\mathrm{~d}, J_{\mathrm{CP}}=\right.$ $138.6 \mathrm{~Hz})\left(\mathrm{PC}_{6} \mathrm{H}_{5}\right) .-{ }^{31} \mathrm{P}-\mathrm{NMR}\left(\mathrm{C}_{6} \mathrm{D}_{6}\right): \delta=21.5$.

(Diphenylphosphinyloxymethyl)diphenylsilan (15a): Darstellung analog zur Synthese von 14a durch Umsetzung von $4.80 \mathrm{~g}(22.4$ mmol) $37 \mathrm{mit} 2.40 \mathrm{~g}(23.7 \mathrm{mmol})$ Triethylamin und $5.10 \mathrm{~g}(21.6$ mmol) Diphenylphosphinsäurechlorid in $20 \mathrm{ml}$ Toluol; Ausb. $7.30 \mathrm{~g}(82 \%)$, weiße Kristalle, Schmp. (Zers.) $80-85^{\circ} \mathrm{C}$. - ${ }^{1} \mathrm{H}-\mathrm{NMR}$ $\left(\mathrm{C}_{6} \mathrm{D}_{6}\right): \delta=4.27\left(\mathrm{dd},{ }^{3} J_{\mathrm{HH}}=2.9 \mathrm{~Hz},{ }^{3} J_{\mathrm{HP}}=5.9 \mathrm{~Hz}, 2 \mathrm{H} ; \mathrm{SiCH}_{2} \mathrm{O}\right)$, $5.15\left(\mathrm{t},{ }^{3} J_{\mathrm{HH}}=2.8 \mathrm{~Hz}, 1 \mathrm{H} ; \mathrm{SiH}\right), 7.0-7.9\left(\mathrm{~m}, 20 \mathrm{H} ; \mathrm{SiC}_{6} \mathrm{H}_{5}, \mathrm{PC}_{6} \mathrm{H}_{5}\right)$. - ${ }^{13} \mathrm{C}-\mathrm{NMR}\left(\mathrm{C}_{6} \mathrm{D}_{6}\right): \delta=53.7\left(\mathrm{~d},{ }^{2} J_{\mathrm{CP}}=8.5 \mathrm{~Hz} ; \mathrm{SiCH}_{2} \mathrm{O}\right), 128.4$, $128.6\left(\mathrm{~d}, J_{\mathrm{CP}}=12.2 \mathrm{~Hz}\right), 130.4,131.5,131.9\left(\mathrm{~d}, J_{\mathrm{CP}}=2.6 \mathrm{~Hz}\right), 132.1$ $\left(\mathrm{d}, J_{\mathrm{CP}}=9.9 \mathrm{~Hz}\right), 134.8\left(\mathrm{~d}, J_{\mathrm{CP}}=134.8 \mathrm{~Hz}\right)$ und $135.8\left(\mathrm{SiC}_{6} \mathrm{H}_{5}\right.$, $\mathrm{PC}_{6} \mathrm{H}_{5}$ ). - ${ }^{31} \mathrm{P}-\mathrm{NMR}\left(\mathrm{C}_{6} \mathrm{D}_{6}\right): \delta=32.5$. - CI-MS (pos.; $\mathrm{NH}_{3}$, $\left.120^{\circ} \mathrm{C}\right): m / z(\%)=415(21)\left[M^{+}+\mathrm{H}\right], 337(100)\left[M^{+}-\mathrm{C}_{6} \mathrm{H}_{5}\right]$. $\mathrm{C}_{25} \mathrm{H}_{23} \mathrm{O}_{2}$ PSi (414.5) Ber. C 72.44 H 5.59 Gef. C 72.7 H 5.9

(Diphenylphosphinyloxy)methyldiphenylsilan (15b): Darstellung durch Erhitzen einer $0.4 \mathrm{M}$ Lösung von $15 \mathrm{a}$ in Toluol $\left(100^{\circ} \mathrm{C}, 3 \mathrm{~h}\right)$ und nachfolgendes Verdampfen des Lösungsmittels; farblose, nicht ganz einheitliche Flüssigkeit. - 'H-NMR $\left(\mathrm{C}_{6} \mathrm{D}_{6}\right): \delta=0.78(\mathrm{~s}, 3 \mathrm{H}$; $\left.\mathrm{SiCH}_{3}\right), 7.1-7.9\left(\mathrm{~m}, 20 \mathrm{H} ; \mathrm{SiC}_{6} \mathrm{H}_{5}, \mathrm{PC}_{6} \mathrm{H}_{5}\right) .-{ }^{31} \mathrm{P}-\mathrm{NMR}\left(\mathrm{C}_{6} \mathrm{D}_{6}\right): \delta=$ 23.2.

(1-Acetoxyethyl)bis[(trimethylsilyl)methyl]silan (16a): Darstellung analog zur Synthese von $4 \mathrm{a}$ durch Umsetzung von $4.97 \mathrm{~g}(20.0$ mmol) 43 mit $2.24 \mathrm{~g}(21.9 \mathrm{mmol})$ Essigsäurcanhydrid (Reaktionsdauer $2 \mathrm{~h}$ ); Ausb. $3.30 \mathrm{~g} \mathrm{(57 \% )} \mathrm{einer} \mathrm{farblosen} \mathrm{Flüssigkeit,} \mathrm{Sdp.}$ $60^{\circ} \mathrm{C} / 0.07$ Torr. - 'H-NMR $\left(\mathrm{CDCl}_{3}\right): \delta=-0.35-0.15(\mathrm{~m}, 4 \mathrm{H}$; $\left.\mathrm{SiCH}_{2} \mathrm{Si}\right),-0.01\left(\mathrm{~s}, 9 \mathrm{H} ; \mathrm{SiCH}_{3}\right), 0.00\left(\mathrm{~s}, 9 \mathrm{H} ; \mathrm{SiCH}_{3}\right), 1.23\left(\mathrm{~d},{ }^{3} \mathrm{HHH}_{\mathrm{HH}}=\right.$ $\left.7.4 \mathrm{~Hz}, 3 \mathrm{H} ; \mathrm{SiCHCH}_{3}\right), 1.74\left[\mathrm{~s}, 3 \mathrm{H} ; \mathrm{C}(\mathrm{O}) \mathrm{CH}_{3}\right], 4.05(\mathrm{~m}, 1 \mathrm{H} ; \mathrm{SiH})$, $4.67\left(\mathrm{qd},{ }^{3} J_{\mathrm{HH}}=7.4 \mathrm{~Hz},{ }^{3} \mathrm{~J}_{\mathrm{HH}}=1.4 \mathrm{~Hz}, 1 \mathrm{H} ; \mathrm{SiCHCH}\right) .-{ }^{13} \mathrm{C}-$ NMR $\left(\mathrm{CDCl}_{3}\right): \delta=-1.8\left(\mathrm{SiCH}_{2} \mathrm{Si}\right),-1.6\left(\mathrm{SiCH}_{2} \mathrm{Si}\right), 0.88\left(\mathrm{SiCH}_{3}\right)$, $0.91\left(\mathrm{SiCH}_{3}\right), 16.8(\mathrm{SiCHCH}), 20.9\left[\mathrm{C}(\mathrm{O}) \mathrm{CH}_{3}\right], 63.5\left(\mathrm{SiCHCH}_{3}\right)$, 170.0 (CO). - EI-MS: $m / z(\%)=290(1)\left[M^{+}\right], 275(5)\left[M^{+}-\right.$ $\left.\mathrm{CH}_{3}\right], 203(100)\left[M^{+}-\mathrm{CH}\left(\mathrm{CH}_{3}\right) \mathrm{OC}(\mathrm{O}) \mathrm{CH}_{3}\right], 73(78)\left[\mathrm{Si}\left(\mathrm{CH}_{3}\right)_{3}^{+}\right]$

\section{$\mathrm{C}_{12} \mathrm{H}_{30} \mathrm{O}_{2} \mathrm{Si}_{3}$ (290.6) Ber. C 49.59 H 10.40 Si 28.99} Gef. C 49.3 H 10.5 Si 29.2

Acetoxyethylbis[(trimethylsilyl)methyl]silan (16b): Darstellung durch Erhitzen von $16 \mathrm{a}\left(135^{\circ} \mathrm{C}, 60 \mathrm{~h}\right)$; farblose Flüssigkeit. $-{ }^{1} \mathrm{H}$ $\operatorname{NMR}\left(\mathrm{C}_{6} \mathrm{D}_{6}\right): \delta=0.03\left(\delta_{\mathrm{A}}\right)$ und $0.07\left(\delta_{\mathrm{B}}\right)\left(\mathrm{AB}-\mathrm{Teil}, J_{\mathrm{AB}}=13.4 \mathrm{~Hz}\right.$, $\left.4 \mathrm{H} ; \mathrm{SiCH}_{2} \mathrm{Si}\right), 0.07\left(\mathrm{~s}, 18 \mathrm{H} ; \mathrm{SiCH}_{3}\right), 0.79\left(\mathrm{q},{ }^{3} J_{\mathrm{HH}}=7.9 \mathrm{~Hz}, 2 \mathrm{H}\right.$; $\left.\mathrm{SiCH}_{2} \mathrm{CH}_{3}\right), 0.98\left(\mathrm{t},{ }^{3} J_{\mathrm{HH}}=7.9 \mathrm{~Hz}, 3 \mathrm{H} ; \mathrm{SiCH}_{2} \mathrm{CH}_{3}\right), 1.77[\mathrm{~s}, 3 \mathrm{H}$; $\left.\mathrm{C}(\mathrm{O}) \mathrm{CH}_{3}\right] .-{ }^{13} \mathrm{C}-\mathrm{NMR}\left(\mathrm{C}_{6} \mathrm{D}_{6}\right): \delta=1.2\left(\mathrm{SiCH}_{3}\right), 3.2\left(\mathrm{SiCH}_{2} \mathrm{Si}\right), 6.9$ $\left(\mathrm{SiCH}_{2} \mathrm{CH}_{3}\right), 9.8\left(\mathrm{SiCH}_{2} \mathrm{CH}_{3}\right), 22.5\left[\mathrm{C}(\mathrm{O}) \mathrm{CH}_{3}\right], 170.2(\mathrm{CO}) .-\mathrm{EI}-$ MS: $m / z(\%)=275(26)\left[M^{+}-\mathrm{CH}_{3}\right], 261(31)\left[M^{+}-\mathrm{CH}_{2} \mathrm{CH}_{3}\right]$, $203(100)\left[M^{+}-\mathrm{CH}_{2} \mathrm{Si}\left(\mathrm{CH}_{3}\right)_{3}\right]$.

$\mathrm{C}_{12} \mathrm{H}_{30} \mathrm{O}_{2} \mathrm{Si}_{3}$ (290.6) Ber. C 49.59 H 10.40 Gef. C 49.7 H 10.4

Benzyl(chlorformyloxymethyl)methylsilan (17a): In eine Lösung von $8.00 \mathrm{~g}$ (48.1 mmol) 29 in $30 \mathrm{ml}$ Phosgen-gesättigtem Benzol wurde innerhalb von $3 \mathrm{~h}$ unter Rühren bei $6^{\circ} \mathrm{C}$ ein schwacher Phosgen-Strom geleitet. Anschließend rührte man noch $30 \mathrm{~min}$ bei Raumtemp., entfernte durch Einleiten eines Stickstoffstromes das überschüssige Phosgen und den gebildeten Chlorwasserstoff, befreite unter vermindertem Druck von den leichtflüchtigen Bestandteilen und unterwarf den Rückstand einer mehrmaligen schonenden Vakuum-Destillation über eine Vigreux-Kolonne; Ausb. $4.10 \mathrm{~g}$ (37\%) einer farblosen Flüssigkeit, Sdp. $56^{\circ} \mathrm{C} / 0.15$ Torr. - 'H-NMR $\left(\mathrm{CDCl}_{3}\right): \delta=0.19\left(\mathrm{~d},{ }^{3} J_{\mathrm{HH}}=3.7 \mathrm{~Hz} ; \mathrm{SiCH}_{3}\right), 2.29\left(\mathrm{~d},{ }^{3} J_{\mathrm{HH}}=\right.$ $3.4 \mathrm{~Hz}, 2 \mathrm{H}$; $\mathrm{SiCH}_{2} \mathrm{C}$ ), $4.0-4.2\left(\mathrm{~m}, 3 \mathrm{H}\right.$; $\mathrm{SiH}$ und $\mathrm{SiCH}_{2} \mathrm{O}$ ), 7.0-7.3 (m, 5H; $\left.\mathrm{CC}_{6} \mathrm{H}_{5}\right) .-{ }^{13} \mathrm{C}-\mathrm{NMR}\left(\mathrm{CDCl}_{3}\right): \delta=-8.0\left(\mathrm{SiCH}_{3}\right), 20.7$ $\left(\mathrm{SiCH}_{2} \mathrm{C}\right), 63.2\left(\mathrm{SiCH}_{2} \mathrm{O}\right), 125.1,128.2,128.8$ und $137.7\left(\mathrm{CC}_{6} \mathrm{H}_{5}\right)$, 151.7 (CO). - EI-MS: $m / z(\%)=137(100)\left[M^{+}-\mathrm{CH}_{2} \mathrm{C}_{6} \mathrm{H}_{5}\right]$, $135(6)\left[M^{+}-\mathrm{CH}_{2} \mathrm{OC}(\mathrm{O}) \mathrm{Cl}\right]$.

$$
\begin{aligned}
& \mathrm{C}_{10} \mathrm{H}_{13} \mathrm{ClO}_{2} \mathrm{Si}(228.8) \\
& \text { Ber. C } 52.51 \text { H } 5.73 \mathrm{Cl} 15.50 \text { Si } 12.28 \\
& \text { Gef. C } 52.3 \text { H } 6.0 \text { Cl } 15.1 \text { Si } 12.3
\end{aligned}
$$

Benzylchlordimethylsilan (17c): Darstellung durch Erhitzen von $17 \mathbf{a}\left(135^{\circ} \mathrm{C}, 36 \mathrm{~h}\right)$; farblose Flüssigkeit. - 'H-NMR $\left(\mathrm{CDCl}_{3}\right): \delta=$ 0.40 (s, 6 H; $\left.\mathrm{SiCH}_{3}\right), 2.42$ (s, $\left.2 \mathrm{H} ; \mathrm{SiCH}_{2} \mathrm{C}\right), 7.0-7.4\left(\mathrm{~m}, 5 \mathrm{H} ; \mathrm{CC}_{6} \mathrm{H}_{5}\right)$. $-{ }^{13} \mathrm{C}$-NMR $\left(\mathrm{CDCl}_{3}\right): \delta=1.2\left(\mathrm{SiCH}_{3}\right), 28.9\left(\mathrm{SiCH}_{2} \mathrm{C}\right), 125.0,128.5$, 128.7 und $137.1\left(\mathrm{CC}_{6} \mathrm{H}_{5}\right)$. - EI-MS: $m / z(\%)=184(78)\left[M^{+}\right], 149$ (10) $\left[M^{+}-\mathrm{Cl}\right], 93(100)\left[M^{+}-\mathrm{CH}_{2} \mathrm{C}_{6} \mathrm{H}_{5}\right]$.

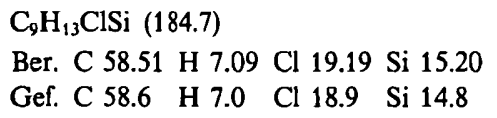

(Chlorformyloxymethyl)methyl[(trimethylsilyl)methyl]silan (18a): Darstellung analog zur Synthese von 17a durch Umsetzung von $6.36 \mathrm{~g}$ (39.2 mmol) $35 \mathrm{mit}$ Phosgen; Ausb. $4.90 \mathrm{~g}$ (56\%) einer farblosen Flüssigkeit, Sdp. $39^{\circ} \mathrm{C} / 0.06$ Torr. - ${ }^{1} \mathrm{H}-\mathrm{NMR}\left(\mathrm{CDCl}_{3}\right)$ : $\delta=-0.18\left(\delta_{\mathrm{A}}\right)$ und $-0.11\left(\delta_{\mathrm{B}}\right)$ [AB-Teil des ABX-Systems Si$\left(\mathrm{H}_{\mathrm{X}}\right) \mathrm{CH}_{\mathrm{A}} \mathrm{H}_{\mathrm{B}} \mathrm{Si}, J_{\mathrm{AB}}=13.9 \mathrm{~Hz}, J_{\mathrm{AX}}=5.2 \mathrm{~Hz}, J_{\mathrm{BX}}=3.1 \mathrm{~Hz}, 2 \mathrm{H}$; 
$\left.\mathrm{SiCH}_{2} \mathrm{Si}\right], 0.05\left[\mathrm{~s}, 9 \mathrm{H} ; \mathrm{Si}\left(\mathrm{CH}_{3}\right)_{3}\right], 0.20\left(\mathrm{~d},{ }^{3} J_{\mathrm{HH}}=3.7 \mathrm{~Hz}, 3 \mathrm{H}\right.$; $\left.\mathrm{SiCH}_{3}\right), 4.1-4.2\left(\mathrm{~m}, 3 \mathrm{H}\right.$; $\mathrm{SiH}$ und $\left.\mathrm{SiCH}_{2} \mathrm{O}\right) .-{ }^{13} \mathrm{C}-\mathrm{NMR}\left(\mathrm{CDCl}_{3}\right)$ : $\delta=-5.2\left(\mathrm{SiCH}_{3}\right),-2.2\left(\mathrm{SiCH}_{2} \mathrm{Si}\right), 0.6\left[\mathrm{Si}\left(\mathrm{CH}_{3}\right)_{3}\right], 65.5\left(\mathrm{SiCH}_{2} \mathrm{O}\right)$, 151.7 (CO). - EI-MS: $m / z(\%)=209(4)\left[M^{+}-\mathrm{CH}_{3}\right], 145(38)$ $\left[M^{+}-\mathrm{OC}(\mathrm{O}) \mathrm{Cl}\right], 137(68)\left[M^{+}-\mathrm{CH}_{2} \mathrm{Si}\left(\mathrm{CH}_{3}\right)_{3}\right], 131$ (100) $\left[M^{+}-\mathrm{CH}_{2} \mathrm{OC}(\mathrm{O}) \mathrm{Cl}\right]$.

$$
\mathrm{C}_{7} \mathrm{H}_{17} \mathrm{ClO}_{2} \mathrm{Si}_{2} \text { (224.8) Ber. C } 37.40 \mathrm{H} 7.62 \mathrm{Cl} 15.77
$$
Gef. C $37.4 \quad$ H $7.5 \quad$ Cl 16.1

Chlordimethyl/(trimethylsilyl)methyl]silan (18c): Darstellung durch Erhitzen von $18 \mathrm{a}\left(135^{\circ} \mathrm{C}, 40 \mathrm{~h}\right)$; farblose Flüssigkeit. - 'HNMR $\left(\mathrm{CDCl}_{3}\right): \delta=0.08\left[\mathrm{~s}, 9 \mathrm{H} ; \mathrm{Si}\left(\mathrm{CH}_{3}\right)_{3}\right], 0.15\left(\mathrm{~s}, 2 \mathrm{H} ; \mathrm{SiCH}_{2} \mathrm{C}\right)$, $0.43\left[\mathrm{~s}, 6 \mathrm{H} ; \mathrm{Si}\left(\mathrm{CH}_{3}\right)_{2}\right] .-{ }^{13} \mathrm{C}-\mathrm{NMR}\left(\mathrm{CDCl}_{3}\right): \delta=1.1\left[\mathrm{Si}\left(\mathrm{CH}_{3}\right)_{3}\right]$, $4.7\left(\mathrm{Si}\left(\mathrm{CH}_{3}\right)_{2}\right), 7.8\left(\mathrm{SiCH}_{2} \mathrm{C}\right) .-$ EI-MS: $m / 2(\%)=180(28)\left[M^{+}\right]$ $165(100)\left[M^{+}-\mathrm{CH}_{3}\right], 93(8)\left[M^{+}-\mathrm{CH}_{2} \mathrm{Si}\left(\mathrm{CH}_{3}\right)_{3}\right], 73(50)$ $\left[\mathrm{Si}\left(\mathrm{CH}_{3}\right)_{3}^{+}\right]$.

$$
\begin{array}{llllll}
\mathrm{C}_{6} \mathrm{H}_{17} \mathrm{ClSi}_{2} \text { (180.8) } & \text { Ber. C } 39.85 & \mathrm{H} 9.48 & \mathrm{Cl} 19.61 \\
& \text { Gef. C } 40.1 & \text { H } 9.8 & \text { Cl } 19.6
\end{array}
$$

(Chlorformyloxymethyl)bis/(trimethylsilyl)methyl/silan (19a): Darstellung analog zur Synthese von 17 a durch Umsetzung von $3.20 \mathrm{~g}$ (13.6 mmol) $36 \mathrm{mit}$ Phosgen; Ausb. $2.70 \mathrm{~g} \mathrm{(67 \% )} \mathrm{einer} \mathrm{farb-}$ losen Flüssigkeit, Sdp. $53^{\circ} \mathrm{C} / 0.1$ Torr. $-{ }^{1} \mathrm{H}-\mathrm{NMR}\left(\mathrm{CDCl}_{3}\right): \delta=$ $-0.16\left(\delta_{\mathrm{A}}\right)$ und $-0.11\left(\delta_{\mathrm{B}}\right)$ [AB-Tcil des ABX-Systems $\mathrm{Si}\left(\mathrm{H}_{\mathrm{X}}\right)$ $\mathrm{CH}_{\mathrm{A}} \mathrm{H}_{\mathrm{B}} \mathrm{Si}, J_{\mathrm{AB}}=14.0 \mathrm{~Hz}, J_{\mathrm{AX}}=3.6 \mathrm{~Hz}, J_{\mathrm{BX}}=4.2 \mathrm{~Hz}, 4 \mathrm{H} ; \mathrm{Si}-$ $\left.\mathrm{CH}_{2} \mathrm{Si}\right], 0.06\left(\mathrm{~s}, 18 \mathrm{H} ; \mathrm{SiCH}_{3}\right), 4.12\left(\mathrm{~d},{ }^{3} \mathrm{~J}_{\mathrm{HH}}=2.5 \mathrm{~Hz}, 2 \mathrm{H} ; \mathrm{SiCH}_{2} \mathrm{O}\right)$, $4.08(\mathrm{~m}, 1 \mathrm{H} ; \mathrm{SiH}) .-{ }^{13} \mathrm{C}$-NMR $\left(\mathrm{CDCl}_{3}\right): \delta=-1.2\left(\mathrm{SiCH}_{2} \mathrm{Si}\right), 0.6$ $\left(\mathrm{SiCH}_{3}\right), 65.7\left(\mathrm{SiCH}_{2} \mathrm{O}\right), 151.7(\mathrm{CO})$ - - EI-MS: $m / z(\%)=217(8)$ $\left[M^{+}-\mathrm{OC}(\mathrm{O}) \mathrm{Cl}\right], 209\left[M^{+}-\mathrm{CH}_{2} \mathrm{Si}\left(\mathrm{CH}_{3}\right)_{3}\right], 203(58)\left[M^{+}-\right.$ $\left.\mathrm{CH}_{2} \mathrm{OC}(\mathrm{O}) \mathrm{Cl}\right], 73(100)\left[\mathrm{Si}\left(\mathrm{CH}_{3}\right)_{3}^{+}\right]$.

$$
\begin{aligned}
& \mathrm{C}_{10} \mathrm{H}_{25} \mathrm{ClO}_{2} \mathrm{Si}_{3}(297.0) \\
& \text { Ber. C } 40.44 \mathrm{H} 8.48 \mathrm{Cl} 11.94 \mathrm{Si} 28.37 \\
& \text { Gef. C } 40.4 \quad \mathrm{H} 8.6 \quad \mathrm{Cl} 12.1 \quad \mathrm{Si} 28.2
\end{aligned}
$$

Chlormethylbis/(trimethylsilyl)methyl]silan (19c): Darstellung durch Erhitzen von $19 \mathrm{a}\left(135^{\circ} \mathrm{C}, 30 \mathrm{~h}\right)$; farblose Flüssigkeit. - ${ }^{1} \mathrm{H}$ $\operatorname{NMR}\left(\mathrm{CDCl}_{3}\right): \delta=0.08\left[\mathrm{~s}, 18 \mathrm{H} ; \mathrm{Si}\left(\mathrm{CH}_{3}\right)_{3}\right], 0.09\left(\delta_{\mathrm{A}}\right)$ und $0.15\left(\delta_{\mathrm{B}}\right)$ (AB-System, $\left.J_{A B}=13.6 \mathrm{~Hz}, 4 \mathrm{H} ; \mathrm{SiCH}_{2} \mathrm{Si}\right), 0.43\left(\mathrm{~s}, 3 \mathrm{H} ; \mathrm{SiCH}_{3}\right.$ ). ${ }^{13} \mathrm{C}-\mathrm{NMR}\left(\mathrm{CDCl}_{3}\right): \delta=1.2\left[\mathrm{Si}\left(\mathrm{CH}_{3}\right)_{3}\right], 5.9\left(\mathrm{SiCH}_{3}\right), 9.1\left(\mathrm{SiCH}_{2} \mathrm{Si}\right)$. - EI-MS: $m / z(\%)=252(3)\left[M^{+}\right], 237(100)\left[M^{+}-\mathrm{CH}_{3}\right]$.

$\mathrm{C}_{9} \mathrm{H}_{25} \mathrm{ClSi}_{3}$ (253.0) Ber. C $42.73 \mathrm{H} 9.96 \mathrm{Cl} 14.01$ Gef. C 43.1 H $10.1 \quad \mathrm{Cl} 14.1$

(Chlorformyloxymethyl)[(trimethylsilyl)methyl]silan (20a): Darsteilung gemäß Lit. ${ }^{\left[{ }^{[t]} \text {. }\right.}$

\author{
(Chlormethyl)dimethoxymethylsilan (22): Darstellung gemäß \\ Lit. ${ }^{[2]}$.
}

(Chlormethyl)methoxymethyl( 1-naphthyl)silan (23): Zu einer Lösung von $75.8 \mathrm{~g}(0.49 \mathrm{~mol}) 22$ in $200 \mathrm{ml}$ Diethylether wurde bei Raumtemp. unter Rühren innerhalb von $2 \mathrm{~h}$ eine aus $12.2 \mathrm{~g}(0.50$ mol) Magnesium und $103.5 \mathrm{~g}(0.50 \mathrm{~mol}) 1-$ Naphthylbromid in $560 \mathrm{ml}$ Diethylether/Toluol $(1.15 / 1, \mathrm{v} / \mathrm{v})$ hergestellte Grignard-Lösung getropft. Nach beendeter Zugabe rührte man $20 \mathrm{~h}$ unter Rückfluß, filtrierte den Niederschlag ab, befreite das Filtrat unter vermindertem Druck vom Lösungsmittel und nahm den Rückstand in 11 Petrolether auf. Es wurde erneut filtriert, das Filtrat unter vermindertem Druck vom Lösungsmittel befreit und der Rückstand im Vakuum fraktionierend über eine Vigreux-Kolonne destilliert; Ausb. $73.7 \mathrm{~g}(60 \%)$ einer farblosen Flüssigkeit, Sdp. $112^{\circ} \mathrm{C} / 0.1$ Torr. - 'H-NMR $\left(\mathrm{CDCl}_{3}\right): \delta=0.69\left(\mathrm{~s}, 3 \mathrm{H} ; \mathrm{SiCH}_{3}\right), 3.14\left(\delta_{\mathrm{A}}\right)$ und 3.22 $\left(\delta_{\mathrm{B}}\right)\left(\mathrm{AB}-\mathrm{System}, J_{\mathrm{AB}}=14.1 \mathrm{~Hz}, 2 \mathrm{H} ; \mathrm{SiCH}_{2} \mathrm{Cl}\right), 3.57\left(\mathrm{~s}, 3 \mathrm{H} ; \mathrm{OCH}_{3}\right)$, 7.4-8.2 (m, 7 H; $\left.\mathrm{SiC}_{10} \mathrm{H}_{7}\right) .-{ }^{13} \mathrm{C}-\mathrm{NMR}\left(\mathrm{CDCl}_{3}\right): \delta=-4.3\left(\mathrm{SiCH}_{3}\right)$, $29.1\left(\mathrm{SiCH}_{2} \mathrm{Cl}\right), 51.4\left(\mathrm{OCH}_{3}\right), 125.0,125.8,126.5,127.6,129.1,131.2$, 131.8, 133.3, 134.7 und $136.9\left(\mathrm{SiC}_{10} \mathrm{H}_{7}\right) .-{ }^{29} \mathrm{Si}-\mathrm{NMR}\left(\mathrm{C}_{6} \mathrm{D}_{6}\right): \delta=$
3.5. - EI-MS: $m / z(\%)=250(3)\left[M^{+}\right], 201(63)\left[M^{+}-\mathrm{CH}_{2} \mathrm{Cl}\right]$, $141(100)\left[\mathrm{C}_{11} \mathrm{H}_{9}^{+}\right]$.

$$
\mathrm{C}_{13} \mathrm{H}_{15} \mathrm{ClOSi} \text { (250.8) Ber. C } 62.26 \mathrm{H} 6.03 \mathrm{Si} 11.20
$$$$
\text { Gef. C } 62.2 \text { H } 6.4 \text { Si } 10.9
$$

(Acetoxymethyl)methoxymethyl(1-naphthyl)silan (24): Ein Gemisch aus $10.5 \mathrm{~g}$ (41.9 mmol) $23,3.50 \mathrm{~g}(42.7 \mathrm{mmol})$ Natriumacetat und $60 \mathrm{ml}$ Dimethylformamid wurde $3 \mathrm{~h}$ unter Rückfluß gerührt. Der Niederschlag wurde abfiltriert, das Lösungsmittel abdestillicrt und der Rückstand in Petrolether/Diethylether $(3 / 1, v / v)$ aufgenommen. Dann filtrierte man erneut, befreite das Filtrat unter vermindertem Druck vom Lösungsmittel und destillierte den Rückstand im Kugelrohrverdampfer $\left(130-210^{\circ} \mathrm{C} / 0.001\right.$ Torr); Ausb. $9.80 \mathrm{~g}$ $(85 \%)$ einer farblosen Flüssigkeit. - ${ }^{1} \mathrm{H}-\mathrm{NMR}\left(\mathrm{CDCl}_{3}\right): \delta=0.62$ (s, $\left.3 \mathrm{H} ; \mathrm{SiCH}_{3}\right), 1.99\left(\mathrm{~s}, 3 \mathrm{H} ; \mathrm{CCH}_{3}\right), 3.53\left(\mathrm{~s}, 3 \mathrm{H} ; \mathrm{OCH}_{3}\right), 4.16\left(\delta_{\mathrm{A}}\right)$ und $4.20\left(\delta_{\mathrm{B}}\right)\left(\mathrm{AB}-\mathrm{System}, J_{\mathrm{AB}}=14.8 \mathrm{~Hz}, 2 \mathrm{H} ; \mathrm{SiCH}_{2} \mathrm{O}\right), 7.4-8.3$ $\left(\mathrm{m}, 7 \mathrm{H} ; \mathrm{SiC}_{10} \mathrm{H}_{7}\right) .-{ }^{13} \mathrm{C}-\mathrm{NMR}\left(\mathrm{CDCl}_{3}\right): \delta=-4.1\left(\mathrm{SiCH}_{3}\right), 20.7$ $\left(\mathrm{CCH}_{3}\right), 51.1\left(\mathrm{OCH}_{3}\right), 56.0\left(\mathrm{SiCH}_{2} \mathrm{O}\right), 125.0,125.7,126.4,127.8$, 128.9, 131.1, 132.1, 133.3, 134.4 und $136.9\left(\mathrm{SiC}_{10} \mathrm{H}_{7}\right), 171.6(\mathrm{CO})$. ${ }^{29} \mathrm{Si}-\mathrm{NMR}\left(\mathrm{C}_{6} \mathrm{D}_{6}\right): \delta=4.1$. - EI-MS: $m / z(\%)=259(20)\left[M^{+}-\right.$ $\left.\mathrm{CH}_{3}\right], 216(25)\left[M^{+}-\mathrm{CH}_{3}-\mathrm{OCH}_{3}\right], 201(100)\left[M^{+}-\right.$ $\left.\mathrm{CH}_{2} \mathrm{OC}(\mathrm{O}) \mathrm{CH}_{3}\right]$.

$$
\mathrm{C}_{15} \mathrm{H}_{18} \mathrm{O}_{3} \mathrm{Si}(274.4) \quad \text { Ber. C } 65.66 \mathrm{H} 6.61 \mathrm{Si} 10.24
$$
Gef. C 66.2 H 6.9 Si 9.9

(Hydroxymethyl)methyl(1-naphthyl)silan (25): Zu einer Suspension von $6.90 \mathrm{~g}(182 \mathrm{mmol})$ Lithiumaluminiumhydrid in $340 \mathrm{ml}$ Diethylether wurde bei $0^{\circ} \mathrm{C}$ unter Rühren innerhalb von $40 \mathrm{~min}$ eine Lösung von $46.9 \mathrm{~g}$ (171 mmol) 24 in $140 \mathrm{ml}$ Diethylether getropft. Nach beendeter Zugabe rührte man $6 \mathrm{~h}$ unter Rückflu B, tropfte das erkaltete Reaktionsgemisch unter starkem Rühren und Eiskühlung zu $260 \mathrm{ml} 12.5$ proz. Salzsäure, trennte die etherische Lösung $a b$, extrahierte die wäßrige Phase dreimal mit je $80 \mathrm{ml}$ Diethylether, trocknete die vereinigten organischen Extrakte mit $\mathrm{Na}_{2} \mathrm{SO}_{4}$, befreite unter vermindertem Druck vom Lösungsmittel und destillierte den Rückstand im Vakuum fraktionierend über eine Vigreux-Kolonne; Ausb. $23.1 \mathrm{~g}(67 \%)$ einer farblosen Flüssigkeit, Sdp. $104-110^{\circ} \mathrm{C} / 0.001$ Torr. $-{ }^{1} \mathrm{H}-\mathrm{NMR}\left(\mathrm{CDCl}_{3}\right): \delta=0.63$ (d, $\left.{ }^{3} J_{\mathrm{HH}}=3.9 \mathrm{~Hz}, 3 \mathrm{H} ; \mathrm{SiCH}_{3}\right), 1.7$ (s, verbreitert, nicht lagekonstant, $1 \mathrm{H}$; OH), $3.81\left(\delta_{\mathrm{A}}\right)$ und $3.86\left(\delta_{\mathrm{B}}\right)$ [AB-Teil des ABX-Systems Si$\left(\mathrm{H}_{\mathrm{X}}\right) \mathrm{CH}_{\mathrm{A}} \mathrm{H}_{\mathrm{B}} \mathrm{O}, J_{\mathrm{AB}}=14.2 \mathrm{~Hz}, J_{\mathrm{AX}}=2.9 \mathrm{~Hz}, J_{\mathrm{BX}}=2.7 \mathrm{~Hz}, 2 \mathrm{H}$; $\left.\mathrm{SiCH}_{2} \mathrm{O}\right], 4.94(\mathrm{~m}, 1 \mathrm{H} ; \mathrm{SiH}), 7.5-8.2\left(\mathrm{~m}, 7 \mathrm{H} ; \mathrm{SiC}_{10} \mathrm{H}_{7}\right) .-{ }^{13} \mathrm{C}-$ $\operatorname{NMR}\left(\mathrm{CDCl}_{3}\right): \delta=-7.0\left(\mathrm{SiCH}_{3}\right), 53.5\left(\mathrm{SiCH}_{2} \mathrm{O}\right), 125.2,125.7$, 126.2, 127.6, 128.9, 130.5, 132.3, 133.2, 134.7 und $137.2\left(\mathrm{SiC}_{10} \mathrm{H}_{7}\right)$. ${ }^{29} \mathrm{Si}-\mathrm{NMR}\left(\mathrm{C}_{6} \mathrm{D}_{6}\right) . \delta=-20.7$. - EI-MS: $m / z(\%)=202(19)\left[M^{+}\right]$, $171(100)\left[M^{+}-\mathrm{CH}_{2} \mathrm{OH}\right]$

$$
\begin{array}{lllll}
\mathrm{C}_{12} \mathrm{H}_{14} \mathrm{OSi} \text { (202.3) } & \text { Ber. C } 71.24 & \text { H } 6.97 & \text { Si } 13.88 \\
& \text { Gef. C } 71.0 & \text { H } 7.1 & \text { Si } 14.2
\end{array}
$$

Dichlor(chlormethyl)methylsilan (26) stand als Handelsprodukt zur Verfügung.

Benzylchlor(chlormethyl)methylsilan (27): Ein aus $12.2 \mathrm{~g}(0.50$ mol) Magnesium und $63.3 \mathrm{~g}(0.50 \mathrm{~mol})$ Benzylchlorid in $500 \mathrm{ml}$ Diethylether hergestelltes Grignard-Reagenz wurde bei $0^{\circ} \mathrm{C}$ unter starkem Rühren innerhalb von $4 \mathrm{~h}$ zu einer Lösung von $81.8 \mathrm{~g}(0.50$ mol) 26 in $400 \mathrm{ml}$ Petrolether getropft. Nach beendeter Zugabe rührte man $14 \mathrm{~h}$ bei Raumtemp. und $8 \mathrm{~h}$ unter RückfluB, filtrierte den Niederschlag ab, wusch diesen dreimal mit je $50 \mathrm{ml}$ Petrolether, vereinigte Filtrat und Waschlösungen, befreite unter vermindertem Druck vom Lösungsmittel und destillierte den Rückstand im Vakuum fraktionierend über eine Vigreux-Kolonne; Ausb. $78.9 \mathrm{~g}$ (72\%) einer farblosen Flüssigkeit, Sdp. $112^{\circ} \mathrm{C} / 15$ Torr. - 'H-NMR $\left(\mathrm{CDCl}_{3}\right): \delta=0.57\left(\mathrm{~s}, 3 \mathrm{H} ; \mathrm{SiCH}_{3}\right), 2.60\left(\mathrm{~s}, 2 \mathrm{H} ; \mathrm{SiCH}_{2} \mathrm{C}\right), 2.96(\mathrm{~s}, 2 \mathrm{H}$; $\left.\mathrm{SiCH}_{2} \mathrm{Cl}\right), 7.1-7.4\left(\mathrm{~m}, 5 \mathrm{H} ; \mathrm{CC}_{6} \mathrm{H}_{5}\right) .-{ }^{13} \mathrm{C}-\mathrm{NMR}\left(\mathrm{CDCl}_{3}\right): \delta=$ 
-1.9 $\left(\mathrm{SiCH}_{3}\right), 25.1\left(\mathrm{SiCH}_{2} \mathrm{C}\right), 28.7\left(\mathrm{SiCH}_{2} \mathrm{Cl}\right), 125.5,128.69,128.71$ und $135.8\left(\mathrm{CC}_{6} \mathrm{H}_{5}\right)$. - EI-MS: $m / z(\%)=218(25)\left[M^{+}\right], 203(1)$ $\left[M^{+}-\mathrm{CH}_{3}\right], 183(5)\left[M^{+}-\mathrm{Cl}\right], 169(40)\left[M^{+}-\mathrm{CH}_{2} \mathrm{Cl}\right], 91$ (100) $\left[\mathrm{C}_{7} \mathrm{H}_{7}^{+}\right]$.

\section{$\mathrm{C}_{9} \mathrm{H}_{12} \mathrm{Cl}_{2} \mathrm{Si}(219.2)$ Ber. C 49.32 H 5.52 Gel. C 48.9 H 5.5}

Acetoxy (acetoxymethyl) benzylmethylsilan (28): Zu einer Lösung von $65.8 \mathrm{~g}(0.30 \mathrm{~mol}) 27$ und $130 \mathrm{ml}$ Triethylamin in $400 \mathrm{ml}$ Toluol wurde bei Raumtemp. unter starkem Rühren innerhalb von $6.5 \mathrm{~h}$ eine Lösung von $44.4 \mathrm{~g}(0.74 \mathrm{~mol})$ Eisessig in $100 \mathrm{ml}$ Toluol getropft. Anschließend rührte man $10 \mathrm{~h}$ unter Rückfluß, befreite unter vermindertem Druck vom überschüssigen Triethylamin und vom Lösungsmittel, versetzte den Rückstand mit $250 \mathrm{ml}$ Petrolether, filtrierte den Niederschlag ab, wusch diesen zweimal mit je $50 \mathrm{ml}$ Petrolcther, vereinigte Filtrat und Waschlösungen, befreite vom Lösungsmittel und destillierte den Rückstand im Vakuum fraktionierend über eine Vigreux-Kolonne; Ausb. $70.9 \mathrm{~g}(89 \%)$ einer farblosen Flüssigkeit, Sdp. $104^{\circ} \mathrm{C} / 0.5$ Torr. $-{ }^{1} \mathrm{H}-\mathrm{NMR}\left(\mathrm{CDCl}_{3}\right): \delta=0.28$ (s, $\left.3 \mathrm{H} ; \mathrm{SiCH}_{3}\right), 1.956\left(\mathrm{~s}, 3 \mathrm{H} ; \mathrm{CCH}_{3}\right), 1.961\left(\mathrm{~s}, 3 \mathrm{H} ; \mathrm{CCH}_{3}\right), 2.42(\mathrm{~s}$ $2 \mathrm{H} ; \mathrm{SiCH}_{2} \mathrm{C}$ ), 3.81 (s, $\left.2 \mathrm{H} ; \mathrm{SiCH}_{2} \mathrm{O}\right), 7.0-7.2\left(\mathrm{~m}, 5 \mathrm{H} ; \mathrm{CC}_{6} \mathrm{H}_{5}\right)$. ${ }^{13} \mathrm{C}-\mathrm{NMR}\left(\mathrm{CDCl}_{3}\right): \delta=-4.7\left(\mathrm{SiCH}_{3}\right), 19.9\left(\mathrm{CCH}_{3}\right), 22.1\left(\mathrm{CCH}_{3}\right)$, $23.2\left(\mathrm{SiCH}_{2} \mathrm{C}\right), 54.9\left(\mathrm{SiCH}_{2} \mathrm{O}\right), 124.6,128.15,128.20$ und 136.8 $\left(\mathrm{CC}_{6} \mathrm{H}_{5}\right), 171.3(\mathrm{CO}), 171.6(\mathrm{CO})$. - EI-MS: $m / z(\%)=251(1)\left[\mathrm{M}^{+}\right.$ $\left.-\mathrm{CH}_{3}\right], 207$ (10) $\left[M^{+}-\mathrm{OC}(\mathrm{O}) \mathrm{CH}_{3}\right], 193$ (32) $\left[M^{+}-\right.$ $\left.\mathrm{CH}_{2} \mathrm{OC}(\mathrm{O}) \mathrm{CH}_{3}\right], 175(89)\left[M^{+}-\mathrm{CH}_{2} \mathrm{C}_{6} \mathrm{H}_{5}\right], 91(100)\left[\mathrm{C}_{7} \mathrm{H}_{7}^{+}\right]$.

$\mathrm{C}_{13} \mathrm{H}_{18} \mathrm{O}_{4} \mathrm{Si}(266.4)$ Ber. C $58.62 \mathrm{H} 6.81$ Gcf. C 58.4 H 6.7

Benzyl(hydroxymethyl)methylsilan (29): Darstellung analog zur Synthese von 25 durch Umsetzung von $21.3 \mathrm{~g}(80.0 \mathrm{mmol}) 28 \mathrm{mit}$ $6.10 \mathrm{~g}(161 \mathrm{mmol})$ Lithiumaluminiumhydrid in $100 \mathrm{ml}$ Diethylether (10 h Rückfluß); Ausb. $11.5 \mathrm{~g}(86 \%)$ einer farblosen Flüssigkeit, Sdp. $88-90^{\circ} \mathrm{C} / 0.3$ Torr. $-{ }^{1} \mathrm{H}-\mathrm{NMR}\left(\mathrm{CDCl}_{3}\right): \delta=0.09\left(\mathrm{~d},{ }^{3} \mathrm{~J}_{\mathrm{HH}}=\right.$ $3.7 \mathrm{~Hz}, 3 \mathrm{H} ; \mathrm{SiCH}_{3}$ ), 2.0 (s, verbreitert, nicht lagekonstant, $1 \mathrm{H} ; \mathrm{OH}$ ), $2.23\left(\mathrm{~d},{ }^{3} J_{\mathrm{HH}}=3.5 \mathrm{~Hz}, 2 \mathrm{H} ; \mathrm{SiCH}_{2} \mathrm{C}\right), 3.42\left(\mathrm{~d},{ }^{3} \mathrm{~J}_{\mathrm{HH}}=2.6 \mathrm{~Hz}, 2 \mathrm{H}\right.$ $\mathrm{SiCH}_{2} \mathrm{O}$ ), $3.96\left(\mathrm{qtt},{ }^{3} J_{\mathrm{HH}}=3.7 \mathrm{~Hz},{ }^{3} J_{\mathrm{HH}}=3.5 \mathrm{~Hz},{ }^{3} J_{\mathrm{HH}}=2.6 \mathrm{~Hz}\right.$, $1 \mathrm{H} ; \mathrm{SiH}), 7.0-7.3\left(\mathrm{~m}, 5 \mathrm{H} ; \mathrm{CC}_{6} \mathrm{H}_{5}\right) .-{ }^{13} \mathrm{C}-\mathrm{NMR}\left(\mathrm{CDCl}_{3}\right): \delta=$ -8.3 $\left(\mathrm{SiCH}_{3}\right), 20.7\left(\mathrm{SiCH}_{2} \mathrm{C}\right), 52.4\left(\mathrm{SiCH}_{2} \mathrm{O}\right), 124.4,128.1,128.4$ und $139.2\left(\mathrm{CC}_{6} \mathrm{H}_{5}\right) .-\mathrm{EI}-\mathrm{MS}: m / z(\%)=151(5)\left[M^{+}-\mathrm{CH}_{3}\right], 135$ (100) $\left[M^{+}-\mathrm{CH}_{2} \mathrm{OH}\right], 75$ (27) $\left[M^{+}-\mathrm{CH}_{2} \mathrm{C}_{6} \mathrm{H}_{5}\right]$.

$\mathrm{C}_{9} \mathrm{H}_{14} \mathrm{OSi}$ (166.3) Ber. C 65.00 H 8.49 Gef. C 65.2 H 8.5

Dichlor(chlormethyl)[(trimethylsilyl)methyl]silan (30): Darstellung gemäß Lit. ${ }^{[l e]}$.

Chlor(chlormethyl)methyl/(trimethylsilyl)methyl/silan (31): Ein aus $8.80 \mathrm{~g}$ (0.36 mol) Magnesium und $44.2 \mathrm{~g}(0.36 \mathrm{~mol})$ (Chlormethyl)trimethylsilan in $170 \mathrm{ml}$ Diethylether hergestelltes GrignardReagenz wurde bei $0^{\circ} \mathrm{C}$ unter starkem Rühren innerhalb von $2 \mathrm{~h}$ zu einer Lösung von $58.9 \mathrm{~g}(0.36 \mathrm{~mol}) 26$ in $300 \mathrm{ml}$ Diethylether getropft. Anschließend ließ man 8 h unter Rück $\bigwedge u ß$ rühren, filtrierte den Niederschlag ab, wusch diesen zweimal mit je $20 \mathrm{ml}$ Diethylether, vereinigte Filtrat und Waschlösungen und destillierte bei Normaldruck das Lösungsmittel ab. Der Rückstand wurde in $250 \mathrm{ml}$ Petrolether aufgenommen, der resultierende Niederschlag abfiltriert und das Filtrat durch Destillation bei Normaldruck vom Lösungsmittel befreit. Die fraktionierende Destillation des Rückstandes im Vakuum über eine Vigreux-Kolonne lieferte $50.4 \mathrm{~g}$ $(65 \%)$ einer farblosen Flüssigkeit, Sdp. $100-103^{\circ} \mathrm{C} / 50$ Torr). ${ }^{1} \mathrm{H}$-NMR $\left(\mathrm{CDCl}_{3}\right) ; \delta=0.12\left[\mathrm{~s}, 9 \mathrm{H} ; \mathrm{Si}\left(\mathrm{CH}_{3}\right)_{3}\right], 0.26\left(\mathrm{~s}, 2 \mathrm{H} ; \mathrm{SiCH}_{2} \mathrm{Si}\right)$, 0.54 (s, $\left.3 \mathrm{H} ; \mathrm{SiCH}_{3}\right), 2.93$ (s, $\left.2 \mathrm{H} ; \mathrm{SiCH}_{2} \mathrm{Cl}\right) .-{ }^{13} \mathrm{C}-\mathrm{NMR}\left(\mathrm{CDCl}_{3}\right)$ : $\delta=1.1\left[\mathrm{Si}\left(\mathrm{CH}_{3}\right)_{3}\right], 1.3\left(\mathrm{SiCH}_{3}\right), 3.9\left(\mathrm{SiCH}_{2} \mathrm{Si}\right), 31.6\left(\mathrm{SiCH}_{2} \mathrm{Cl}\right)$. EI-MS: $m / z(\%)=214(73)\left[M^{+}\right], 199(4)\left[M^{+}-\mathrm{CH}_{3}\right], 179$ (38) $\left[M^{+}-\mathrm{Cl}\right], 165$ (5) $\left[M^{+}-\mathrm{CH}_{2} \mathrm{Cl}\right], 127(87)\left[M^{+}-\right.$ $\left.\left.\mathrm{CH}_{2} \mathrm{Si}\left(\mathrm{CH}_{3}\right)_{3}\right], 73(100)\left[\mathrm{Si}\left(\mathrm{CH}_{3}\right)_{3}\right)^{+}\right]$.

$\mathrm{C}_{6} \mathrm{H}_{16} \mathrm{Cl}_{2} \mathrm{Si}_{2}$ (215.3) Ber. C 33.48 H 7.49 Gef. C 33.0 H 7.4
Chlor(chlormethyl)bis/(trimethylsilyl)methyl]silan (32): Darstellung analog zur Synthese von 31 durch Umsetzung einer aus $3.31 \mathrm{~g}$ $(136 \mathrm{mmol})$ Magnesium und $16.7 \mathrm{~g}(136 \mathrm{mmol})$ (Chlormethyl)trimethylsilan in $200 \mathrm{ml}$ Tetrahydrofuran hergestellten Grignard-Lösung mit einer Lösung von $32.0 \mathrm{~g}$ (136 mmol) 26 in $250 \mathrm{ml}$ Tetrahydrofuran; Ausb. $30.5 \mathrm{~g}(78 \%)$ einer farblosen Flüssigkeit, Sdp. $68^{\circ} \mathrm{C} / 0.4$ Torr. - ' $\mathrm{H}-\mathrm{NMR}\left(\mathrm{CDCl}_{3}\right): \delta=0.12\left(\mathrm{~s}, 18 \mathrm{H} ; \mathrm{SiCH}_{3}\right), 0.23$ $\left(\delta_{\mathrm{A}}\right)$ und $0.28\left(\delta_{\mathrm{B}}\right)\left(\mathrm{AB}-\mathrm{System}, J_{\mathrm{AB}}=13.1 \mathrm{~Hz}, 4 \mathrm{H} ; \mathrm{SiCH}_{2} \mathrm{Si}\right), 2.91$ (s, $\left.2 \mathrm{H} ; \mathrm{SiCH}_{2} \mathrm{Cl}\right) .-{ }^{13} \mathrm{C}-\mathrm{NMR}\left(\mathrm{CDCl}_{3}\right): \delta=1.2\left(\mathrm{SiCH}_{3}\right), 5.1$ $\left(\mathrm{SiCH}_{2} \mathrm{Si}\right), 32.4\left(\mathrm{SiCH}_{2} \mathrm{Cl}\right)$. - EI-MS: $m / z(\%)=271(15)\left[M^{+}-\right.$ $\left.\mathrm{CH}_{3}\right], 237(36)\left[M^{+}-\mathrm{CH}_{2} \mathrm{Cl}\right], 199(70)\left[M^{+}-\mathrm{CH}_{2} \mathrm{Si}\left(\mathrm{CH}_{3}\right)_{3}\right], 73$ (100) $\left[\mathrm{Si}\left(\mathrm{CH}_{3}\right)_{3}^{+}\right]$.

\section{$\mathrm{C}_{9} \mathrm{H}_{24} \mathrm{Cl}_{2} \mathrm{Si}_{3}$ (287.5) Ber. C 37.61 H 8.42 Gef. C 36.9 H 8.4}

Acetoxy (acetoxymethyl)methyl[(trimethylsilyl)methyl/silan (33): $\mathrm{Zu}$ einer Lösung von $25.8 \mathrm{~g}(0.12 \mathrm{~mol}) 31$ in $200 \mathrm{ml}$ Toluol wurde bei Raumtemp. unter starkem Rühren innerhalb von $4 \mathrm{~h}$ eine Lösung von $15.9 \mathrm{~g}(0.26 \mathrm{~mol})$ Eisessig in $25 \mathrm{ml}$ Toluol getropft. Nach beendeter Zugabe rührte man $48 \mathrm{~h}$ unter Rückfluß, filtrierte dann den Niederschlag ab, wusch diesen zweimal mit je $40 \mathrm{ml} \mathrm{Pe}$ trolether, vereinigte Filtrat und Waschlösungen und befreite unter vermindertem Druck vom Lösungsmittel. Die fraktionierende Destillation des Rückstandes im Vakuum über eine Vigreux-Kolonne liefcrte $25.8 \mathrm{~g} \mathrm{(82 \% )}$ einer farblosen Flüssigkeit, Sdp. $67^{\circ} \mathrm{C} / 0.25$ Torr. - 'H-NMR $\left(\mathrm{CDCl}_{3}\right): \delta=0.010\left[\mathrm{~s}, 9 \mathrm{H} ; \mathrm{Si}\left(\mathrm{CH}_{3}\right)_{3}\right], 0.012(\mathrm{~s}$, $\left.2 \mathrm{H} ; \mathrm{SiCH}_{2} \mathrm{Si}\right), 0.29$ (s, $\left.3 \mathrm{H} ; \mathrm{SiCH}_{3}\right), 1.95\left(\mathrm{~s}, 3 \mathrm{H} ; \mathrm{CCH}_{3}\right), 1.96(\mathrm{~s}, 3 \mathrm{H}$; $\left.\mathrm{CCH}_{3}\right), 3.84\left(\mathrm{~s}, 2 \mathrm{H} ; \mathrm{SiCH}_{2} \mathrm{O}\right) .-{ }^{13} \mathrm{C}-\mathrm{NMR}\left(\mathrm{CDCl}_{3}\right): \delta=-1.9$ $\left(\mathrm{SiCH}_{3}\right), 0.9\left[\mathrm{Si}\left(\mathrm{CH}_{3}\right)_{3}\right], 1.5\left(\mathrm{SiCH}_{2} \mathrm{Si}\right), 20.5\left(\mathrm{CCH}_{3}\right), 22.6\left(\mathrm{CCH}_{3}\right)$, $57.0\left(\mathrm{SiCH}_{2} \mathrm{O}\right), 171.5(\mathrm{CO}), 171.6(\mathrm{CO})$. - El-MS: $m / z(\%)=262$ (2) $\left[M^{+}\right], 247(2)\left[M^{+}-\mathrm{CH}_{3}\right], 203(77)\left[M^{+}-\mathrm{OC}(\mathrm{O}) \mathrm{CH}_{3}\right], 189$ (22) $\left[M^{+}-\mathrm{CH}_{2} \mathrm{OC}(\mathrm{O}) \mathrm{CH}_{3}\right]$.

\section{$\mathrm{C}_{10} \mathrm{H}_{22} \mathrm{O}_{4} \mathrm{Si}_{2}$ (262.5) Ber. C $45.76 \mathrm{H} 8.45$ Gef. C $45.5 \mathrm{H} 8.4$}

Acetoxy (acetoxymethyl)bis[(trimethylsilyl)methyl]silan (34): Darstellung analog zur Synthese von 33 durch Umsetzung von $15.5 \mathrm{~g}(53.9 \mathrm{mmol}) 32 \mathrm{mit} 8.00 \mathrm{~g}(133 \mathrm{mmol})$ Eisessig und $25 \mathrm{ml}$ Triethylamin in $100 \mathrm{ml}$ Toluol (76 h Rückfluß); Ausb. $14.3 \mathrm{~g} \mathrm{(79 \% )}$ einer farblosen Flüssigkeit, Sdp. $105^{\circ} \mathrm{C} / 0.1$ Torr. - ${ }^{1} \mathrm{H}-\mathrm{NMR}$ $\left(\mathrm{CDCl}_{3}\right): \delta=0.01$ (s, $\left.18 \mathrm{H} ; \mathrm{SiCH}_{3}\right), 0.08\left(\mathrm{~s}, 4 \mathrm{H} ; \mathrm{SiCH}_{2} \mathrm{Si}\right), 1.99$ (s, $\left.3 \mathrm{H} ; \mathrm{CCH}_{3}\right), 2.00\left(\mathrm{~s}, 3 \mathrm{H} ; \mathrm{CCH}_{3}\right), 3.82\left(\mathrm{~s}, 2 \mathrm{H} ; \mathrm{SiCH}_{2} \mathrm{O}\right) .-{ }^{13} \mathrm{C}-\mathrm{NMR}$ $\left(\mathrm{CDCl}_{3}\right): \delta=1.1\left(\mathrm{SiCH}_{3}\right), 2.7\left(\mathrm{SiCH}_{2} \mathrm{Si}\right), 20.7\left(\mathrm{CCH}_{3}\right), 22.8\left(\mathrm{CCH}_{3}\right)$, $56.8\left(\mathrm{SiCH}_{2} \mathrm{O}\right), 171.2(\mathrm{CO}), 171.4(\mathrm{CO})$. - EI-MS: $m / z(\%)=319$ (3) $\left[M^{+}-\mathrm{CH}_{3}\right], 275(61)\left[M^{+}-\mathrm{OC}(\mathrm{O}) \mathrm{CH}_{3}\right], 261(15)\left[M^{+}-\right.$ $\left.\mathrm{CH}_{2} \mathrm{OC}(\mathrm{O}) \mathrm{CH}_{3}\right], 247$ (3) $\left[M^{+}-\mathrm{CH}_{2} \mathrm{Si}\left(\mathrm{CH}_{3}\right)_{3}\right], 73$ (100) $\left[\mathrm{Si}\left(\mathrm{CH}_{3}\right)_{3}^{+}\right]$.

$$
\mathrm{C}_{13} \mathrm{H}_{30} \mathrm{O}_{4} \mathrm{Si}_{3} \text { (334.6) Ber. C } 46.66 \text { H } 9.04 \text { Gef. C } 46.3 \text { H } 9.1
$$

(Hydroxymethyl)methyl((trimethylsilyl)methyl)silan (35): Darstellung analog zur Synthese von 25 durch Umsetzung von $28.9 \mathrm{~g}$ $(0.11 \mathrm{~mol}) 33 \mathrm{mit} 8.70 \mathrm{~g}(0.23 \mathrm{~mol})$ Lithiumaluminiumhydrid in $250 \mathrm{ml}$ Diethylether (10 h RückfluB); Ausb. $14.6 \mathrm{~g}(82 \%)$ einer farblosen Flüssigkeit, Sdp. $45-50^{\circ} \mathrm{C} / 0.02$ Torr. - ' $\mathrm{H}-\mathrm{NMR}\left(\mathrm{CDCl}_{3}\right)$ : $\delta=-0.27\left(\delta_{\mathrm{A}}\right)$ und $-0.18\left(\delta_{\mathrm{B}}\right)$ [AB-Teil des ABX-Systems Si$\left(\mathrm{H}_{\mathrm{X}}\right) \mathrm{CH}_{\mathrm{A}} \mathrm{H}_{\mathrm{B}} \mathrm{Si}, J_{\mathrm{AB}}=13.8 \mathrm{~Hz}, J_{\mathrm{AX}}=3.3 \mathrm{~Hz}, J_{\mathrm{BX}}=1.6 \mathrm{~Hz}, 2 \mathrm{H}$; $\mathrm{SiCH}_{2} \mathrm{Si}$ ], $0.01\left[\mathrm{~s}, 9 \mathrm{H} ; \mathrm{Si}\left(\mathrm{CH}_{3}\right)_{3}\right], 0.12\left(\mathrm{~d},{ }^{3} J_{\mathrm{HH}}=3.7 \mathrm{~Hz}, 3 \mathrm{H}\right.$; $\left.\mathrm{SiCH}_{3}\right), 2.1$ (s, verbreitert, nicht lagekonstant, $1 \mathrm{H} ; \mathrm{OH}$ ), 3.40 (d, $\left.{ }^{3} J_{\mathrm{HH}}=4.6 \mathrm{~Hz}, 2 \mathrm{H} ; \mathrm{SiCH}_{2} \mathrm{O}\right), 4.00-4.04(\mathrm{~m}, 1 \mathrm{HI} ; \mathrm{SiH}) .-{ }^{13} \mathrm{C}-\mathrm{NMR}$ $\left(\mathrm{CDCl}_{3}\right): \delta=-5.4\left(\mathrm{SiCH}_{3}\right),-2.3\left(\mathrm{SiCH}_{2} \mathrm{Si}\right), 0.7\left[\mathrm{Si}\left(\mathrm{CH}_{3}\right)_{3}\right], 54.7$ $\left(\mathrm{SiCH}_{2} \mathrm{O}\right) .-\mathrm{EI}-\mathrm{MS}: m / z(\%)=147(4)\left[M^{+}-\mathrm{CH}_{3}\right], 131(100)$ $\left[M^{+}-\mathrm{CH}_{2} \mathrm{OH}\right], 75(27)\left[M^{+}-\mathrm{CH}_{2} \mathrm{Si}\left(\mathrm{CH}_{3}\right)_{3}\right]$.

$\mathrm{C}_{6} \mathrm{H}_{18} \mathrm{OSi}_{2}$ (162.4) Ber. C 44.38 H 11.17 Gef. C 43.8 H 11.1

(IIydroxymethyl)bis ( trimethylsilyl)methyl]silan (36): Darstellung analog zur Synthese von 25 durch Umsetzung von $16.5 \mathrm{~g}$ (49.3 mmol) 34 mit $3.85(101 \mathrm{mmol})$ Lithiumaluminiumhydrid in $150 \mathrm{ml}$ 
Diethylether (10 h Rückfluß); Ausb. $9.40 \mathrm{~g}(82 \%)$ einer farblosen Flüssigkeit, Sdp. $69-72^{\circ} \mathrm{C} / 0.1$ Torr. - ' $\mathrm{H}$-NMR $\left(\mathrm{CDCl}_{3}\right): \delta=$ $-0.23\left(\delta_{A}\right)$ und $-0.14\left(\delta_{B}\right)$ [AB-Teil des ABX-Systems $\mathrm{Si}\left(\mathrm{H}_{\mathrm{X}}\right)$ $\mathrm{CH}_{\mathrm{A}} \mathrm{H}_{\mathrm{B}} \mathrm{Si}, J_{\mathrm{AB}}=13.9 \mathrm{~Hz}, J_{\mathrm{AX}}=3.6 \mathrm{~Hz}, J_{\mathrm{BX}}=4.1 \mathrm{~Hz}, 4 \mathrm{H}$ $\mathrm{SiCH}_{2} \mathrm{Si}$ ], 0.04 (s, $\left.18 \mathrm{H} ; \mathrm{SiCH}_{3}\right), 1.4$ (s, verbreitert, nicht lagekonstant, $1 \mathrm{H} ; \mathrm{OH}), 3.43\left(\mathrm{~d},{ }^{3} \mathrm{~J}_{\mathrm{HH}}=2.5 \mathrm{~Hz}, 2 \mathrm{H} ; \mathrm{SiCH}_{2} \mathrm{O}\right), 3.98(\mathrm{~m}, 1 \mathrm{H}$; $\mathrm{SiH}) .-{ }^{13} \mathrm{C}-\mathrm{NMR}\left(\mathrm{CDCl}_{3}\right): \delta=-1.2\left(\mathrm{SiCH}_{2} \mathrm{Si}\right), 0.8\left[\mathrm{Si}\left(\mathrm{CH}_{3}\right)_{3}\right]$, $55.2\left(\mathrm{SiCH}_{2} \mathrm{O}\right) .-$ EI-MS: $m / z(\%)=234(1)\left[M^{+}\right], 203(100)\left[M^{+}\right.$ $\left.-\mathrm{CH}_{2} \mathrm{OH}\right], 147$ (5) $\left[M^{+}-\mathrm{CH}_{2} \mathrm{Si}\left(\mathrm{CH}_{3}\right)_{3}\right]$.

$\mathrm{C}_{9} \mathrm{H}_{26} \mathrm{OSi}_{3}$ (234.6) Ber. C 46.09 H. 11.17 Gef. C 46.2 H 11.0

(Hydroxymethyl)diphenylsilan (37) und (Hydroxymethyl)dimethylsilan (38) wurden gemäß Lit. ${ }^{[3]}$ dargestellt.

Deuterio(hydroxymethyl)dimethylsilan ([D]-38): Darstellung in Analogie zur Synthese von 38 durch Umsetzung von Acetoxy(acetoxymethyl)dimethylsilan ${ }^{[3]}$ mit $\left[D_{4}\right]$-Lithiumaluminiumhydrid in Diethylether und nachfolgende wäßrige Aufarbeitung. - ${ }^{1} \mathrm{H}$ $\operatorname{NMR}\left(\mathrm{CDCl}_{3}\right) ; \delta=0.12\left(1: 1: 1-\mathrm{t},{ }^{3} \mathrm{~J}_{\mathrm{HD}}=0.5 \mathrm{~Hz}, 6 \mathrm{H} ; \mathrm{SiCH}_{3}\right), 1.6$ (br. s, $1 \mathrm{H} ; \mathrm{OH}), 3.46\left(, \mathrm{sc}^{\circ}, 2 \mathrm{H} ; \mathrm{SiCH}_{2} \mathrm{O}\right) .-{ }^{13} \mathrm{C}-\mathrm{NMR}\left(\mathrm{CDCl}_{3}\right): \delta=$ -6.5 $\left(\mathrm{SiCH}_{3}\right), 54.2\left(\mathrm{SiCH}_{2} \mathrm{O}\right)$ - - ${ }^{29} \mathrm{Si}-\mathrm{NMR}\left(\mathrm{C}_{6} \mathrm{D}_{6}\right): \delta=-16.3$ $\left(1: 1: 1-\mathrm{t},{ }^{1} J_{\text {SiD }}=28.3 \mathrm{~Hz}\right) .-$ EI-MS: $m / z(\%)=91(6)\left[M^{+}\right], 60$ (100) $\left[M^{+}-\mathrm{CH}_{2} \mathrm{OH}\right]$.

\section{$\mathrm{C}_{3} \mathrm{H}_{9}$ DOSi (91.2) Ber. C $39.51 \mathrm{H}(\mathrm{D}) 12.15$} Gef. C 39.9 H(D) 11.9

Dichlorbis[(trimethylsilyl)methyl]silan (39): Darstellung gemäB Lit. ${ }^{[4]}$.

(1-Methoxyvinyl)bis[(trimethylsilyl)methyl]silan (41): $\mathrm{Zu}$ einer Lösung von $16.5 \mathrm{ml}(0.22 \mathrm{~mol})$ Methylvinylether $(d=0.7725 \mathrm{~g}$ $\mathrm{cm}^{-3}, 0^{\circ} \mathrm{C}$ ) in $100 \mathrm{ml}$ Tetrahydrofuran wurden bei $-78^{\circ} \mathrm{C}$ unter Rühren innerhalb von $1 \mathrm{~h} 115 \mathrm{ml}$ einer $1.6 \mathrm{M} t$-Butyllithium-Lösung in $n$-Pentan $(=0.18 \mathrm{~mol} t$ - $\mathrm{BuLi})$ getropft. Das Reaktionsgemisch wurde innerhalb von $3 \mathrm{~h}$ auf $0^{\circ} \mathrm{C}$ erwärmt und dann unter Rühren innerhalb von $3 \mathrm{~h}$ zu einer auf $-78^{\circ} \mathrm{C}$ gekühlten Lösung von $41.0 \mathrm{~g}(0.15 \mathrm{~mol}) 39 \mathrm{in} 140 \mathrm{ml}$ Tetrahydrofuran getropft. Anschließend ließ man unter Erwärmen auf Raumtemp. $14 \mathrm{~h}$ rühren, filtrierte den Niederschlag ab, wusch diesen zweimal mit je $50 \mathrm{ml}$ Petrolether, vereinigte Filtrat und Waschlösung und befreite unter vermindertem Druck von den leichtflüchtigen Bestandteilen. Die fraktionierende Destillation des Rückstandes im Vakuum über eine Vigreux-Kolonne lieferte $32.8 \mathrm{~g}$ eines Gemisches (Sdp. $130-132^{\circ} \mathrm{C} /$ 40 Torr), das laut 'H-NMR-Analyse aus $79 \mathrm{mmol} \mathrm{40,22 \textrm {mmol }}$ Bis[1-methoxyvinyl]bis[(trimethylsilyl)methyl]silan und $7.5 \mathrm{mmol}$ $t$-Butyl(1-methoxyvinyl)bis[(trimethyl)methyl]silan bestand. Eine Lōsung dieses Gemisches in $50 \mathrm{ml}$ Diethylether wurde dann bei $0^{\circ} \mathrm{C}$ unter starkem Rühren innerhalb von $1 \mathrm{~h}$ zu einer Suspension von $8.00 \mathrm{~g}(0.21 \mathrm{~mol})$ Lithiumaluminiumhydrid in $200 \mathrm{ml}$ Diethylether getropft. Man rührte $24 \mathrm{~h}$ bei Raumtemp., destillierte das Lösungsmittel bei Normaldruck weitgehend ab, versetzte mit $50 \mathrm{ml}$ Petrolether, filtrierte den Niederschlag ab, wusch diesen mit $50 \mathrm{ml}$ Petrolether, vereinigte Filtrat und Waschlösungen, befreite unter Normaldruck vom Lösungsmittel und destillierte den Rückstand im Vakuum fraktionierend über eine Vigreux-Kolonne; Ausb. $15.5 \mathrm{~g}(40 \%$, bezogen auf eingesetztes 39$)$ einer farblosen Flüssigkeit, Sdp. $135^{\circ} \mathrm{C} / 120$ Torr. - 'H-NMR $\left(\mathrm{CDCl}_{3}\right): \delta=-0.07\left(\delta_{\mathrm{A}}\right)$ und $-0.18\left(\delta_{\mathrm{B}}\right)\left(\mathrm{AB}-\mathrm{Teil}\right.$ eines ABX-Systems, $J_{\mathrm{AB}}=13.8 \mathrm{~Hz}, J_{\mathrm{AX}}=$ $\left.4.5 \mathrm{~Hz}, J_{\mathrm{BX}}=3.4 \mathrm{~Hz}, 4 \mathrm{H} ; \mathrm{SiCH}_{2} \mathrm{Si}\right), 0.02\left(\mathrm{~s}, 18 \mathrm{H} ; \mathrm{SiCH}_{3}\right), 3.50(\mathrm{~s}$ $\left.3 \mathrm{H} ; \mathrm{OCH}_{3}\right), 4.08(\mathrm{~m}, 1 \mathrm{H} ; \mathrm{SiH}), 4.42$ und 4.61 (beide $\mathrm{d},{ }^{2} J_{\mathrm{HH}}=$ $\left.1.9 \mathrm{~Hz}, 2 \mathrm{H} ; \mathrm{SiC}(\mathrm{OR})=\mathrm{CH}_{2}\right) .-{ }^{13} \mathrm{C}-\mathrm{NMR}\left(\mathrm{CDCl}_{3}\right): \delta=0.2(\mathrm{Si}-$ $\left.\mathrm{CH}_{2} \mathrm{Si}\right), 0.7\left(\mathrm{SiCH}_{3}\right), 54.1\left(\mathrm{OCH}_{3}\right), 95.0\left[\mathrm{SiC}(\mathrm{OR})=\mathrm{CH}_{2}\right], 169.4$ $\left[\mathrm{SiC}(\mathrm{OR})=\mathrm{CH}_{2}\right]$. - EI-MS: $m / z(\%)=260(1)\left[M^{+}\right], 259(1)$ $\left[M^{+}-\mathrm{H}\right], 245(100)\left[M^{+}-\mathrm{Si}\left(\mathrm{CH}_{3}\right)_{3}\right], 203(50)\left[M^{+}-\right.$ $\left.\mathrm{C}\left(\mathrm{OCH}_{3}\right)=\mathrm{CH}_{2}\right]$.

$\mathrm{C}_{11} \mathrm{H}_{28} \mathrm{OSi}_{3}(260.6)$ Ber. C 50.70 H 10.83 Gef. C $50.3 \mathrm{H} 11.0$
Acetylbis[(trimethylsilyl)methyl]silan (42): Zu $35 \mathrm{ml}$ Aceton/1 M Salzsäure (4/1, v/v) wurden bei Raumtemp. unter Rühren innerhalb von $15 \mathrm{~min} 13.0 \mathrm{~g}(49.9 \mathrm{mmol}) 41 \mathrm{gctropft}$. Man rührte $1.5 \mathrm{~h}$ bci Raumtemp., versetzte mit $25 \mathrm{ml}$ Diethylether und $25 \mathrm{ml}$ Wasser, trennte die organische Phase $a b$, extrahierte dic wäßrige Lösung mit $20 \mathrm{ml}$ Diethylether, wusch die vereinigten ctherischen Extrakte mit Wasser neutral, trocknete mit $\mathrm{Na}_{2} \mathrm{SO}_{4}$ und destillierte den Rückstand im Vakuum fraktionierend über eine Vigreux-Kolonne; Ausb. $9.35 \mathrm{~g}(76 \%)$ einer farblosen Flüssigkeit, Sdp. $120-123^{\circ} \mathrm{C} /$ 80 Torr. $-{ }^{1} \mathrm{H}-\mathrm{NMR}\left(\mathrm{CDCl}_{3}\right): \delta=-0.20-0.04\left(\mathrm{~m}, 4 \mathrm{H} ; \mathrm{SiCH}_{2} \mathrm{Si}\right)$, $0.02\left(\mathrm{~s}, 18 \mathrm{H} ; \mathrm{SiCH}_{3}\right), 2.30\left(\mathrm{~s}, 3 \mathrm{H} ; \mathrm{CCH}_{3}\right), 4.15(\mathrm{~m}, 1 \mathrm{H} ; \mathrm{SiH}) .-{ }^{13} \mathrm{C}-$ NMR $\left(\mathrm{CDCl}_{3}\right): \delta=-1.6\left(\mathrm{SiCH}_{2} \mathrm{Si}\right), 0.7\left[\mathrm{Si}\left(\mathrm{CH}_{3}\right)_{3}\right], 36.6\left(\mathrm{CCH}_{3}\right)$, $224.3(\mathrm{CO})$. - EI-MS: $m / z(\%)=246(1)\left[M^{+}\right], 245(1)\left[M^{+}-\right.$

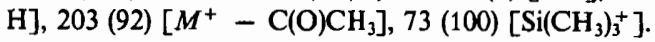

$\mathrm{C}_{10} \mathrm{H}_{26} \mathrm{OSi}_{3}$ (246.6) Ber. C 48.71 H 10.63 Gef. C 48.3 H 10.4

(1-Hydroxyethyl)bis[(trimethylsilyl)methyl]silan (43): Darstellung analog zur Synthese von 25 durch Umsetzung von $7.15 \mathrm{~g}(29.0$ mmol) $42 \mathrm{mit} 2.20 \mathrm{~g}(58.0 \mathrm{mmol})$ Lithiumaluminiumh ydrid in $75 \mathrm{ml}$ Diethylether; Ausb. $5.90 \mathrm{~g}(82 \%)$ einer farblosen Flüssigkeit, Sdp. $100^{\circ} \mathrm{C} / 1$ Torr. $-{ }^{1} \mathrm{H}-\mathrm{NMR}\left(\mathrm{CDCl}_{3}\right): \delta=-0.4-0.1(\mathrm{~m}, 4 \mathrm{H}$; Si$\left.\mathrm{CH}_{2} \mathrm{Si}\right), 0.041$ (s, $\left.9 \mathrm{H} ; \mathrm{SiCH}_{3}\right), 0.044\left(\mathrm{~s}, 9 \mathrm{H} ; \mathrm{SiCH}_{3}\right), 1.1$ (s, verbreitert, nicht lagekonstant, $1 \mathrm{H} ; \mathrm{OH}), 1.28\left[\mathrm{~d},{ }^{3} J_{\mathrm{HH}}=7.4 \mathrm{~Hz}, 3 \mathrm{H}\right.$; $\left.\mathrm{CH}(\mathrm{OH}) \mathrm{CH}_{3}\right], 3.57\left(\mathrm{dq},{ }^{3} \mathrm{~J}_{\mathrm{HH}}=7.4 \mathrm{~Hz},{ }^{3} \mathrm{~J}_{\mathrm{HH}}=1.4 \mathrm{~Hz}, 1 \mathrm{H}\right.$; $\mathrm{CH}(\mathrm{OH}) \mathrm{CH}_{3}$ ), 3.86 (dquint, ${ }^{3} J_{\mathrm{HH}}=5.1 \mathrm{~Hz},{ }^{3} J_{\mathrm{HH}}=1.4 \mathrm{~Hz}, 1 \mathrm{H}$; $\mathrm{SiH}) .-{ }^{13} \mathrm{C}-\mathrm{NMR}\left(\mathrm{CDCl}_{3}\right): \delta=-2.5\left(\mathrm{SiCH}_{2} \mathrm{Si}\right),-2.4\left(\mathrm{SiCH}_{2} \mathrm{Si}\right)$, $0.9\left(\mathrm{SiCH}_{3}\right), 20.2\left[\mathrm{CH}(\mathrm{OH}) \mathrm{CH}_{3}\right], 60.7\left[\mathrm{CH}(\mathrm{OH}) \mathrm{CH}_{3}\right]$ - EI-MS: $m / z(\%)=248(1)\left[M^{+}\right], 203(94)\left[M^{+}-\mathrm{CH}(\mathrm{OH}) \mathrm{CH}_{3}\right], 73(100)$ $\left[\mathrm{Si}\left(\mathrm{CH}_{3}\right)_{3}^{+}\right]$.

$\mathrm{C}_{10} \mathrm{H}_{28} \mathrm{OSi}_{3}$ (248.6) Ber. C $48.32 \mathrm{H} 11.35$ Gef. C $48.4 \mathrm{H} 11.5$

b) 'H-NMR-Untersuchungen zur Kinetik: Zur Bestimmung der kinetischen Parameter (Halbwertszeit $\tau_{1 / 2}$, Geschwindigkeitskonstante $k_{9}^{\mathrm{NMR}}$ ) der thermisch induzierten Umwandlung der Silane $1 \mathrm{a}-20 \mathrm{a}$ wurden jeweils zehn $0.6-1.0 \mathrm{M}$ Lösungen dieser Verbindungen in $C_{6} D_{6}$ bei $-190^{\circ} \mathrm{C}$ unter FeuchtigkeitsausschluB in NMR-Röhrchen eingeschmolzen und sodann in einem thermostatisierbaren Olbad (Gerät D3-19 der Fa. Haake) auf eine definierte Temperatur (siehe Tab. 1) erhitzt. Die Proben wurden dem Thermostaten nach unterschiedlichen Heizzeiten entnommen, sofort in flüssigem Stickstoff abgeschreckt und dann 'H-NMR-spektroskopisch untersucht. Aus dem Integralverhältnis Edukt/Produkt wurde der jeweilige Umsatz $\alpha$ ermittelt. Die Heizzeiten wurden dabei so gewählt, daß der Umsatz im Bereich von $20-80 \%$ lag. Aus dem Umsatz $\alpha$ und der Reaktionszeit $t$ wurde gemä $B$ Gl. (1) für jede Probe die Halbwertszeit $\tau_{1 / 2}$ ermittelt $\left\{\right.$ Gl. (1): $\tau_{1 / 2}=t(\lg 2)[2-$ $\left.\lg (100-\alpha)]^{-1}\right\}$. Aus diesen Einzelwerten wurde dann der Mittelwert für $\tau_{1 / 2}$ bestimmt, wobei sich die Einzelwerte um maximal $8 \%$ voneinander unterschieden. Aus den so bestimmten Mittelwerten von $\tau_{1 / 2}$ wurden gemäB Gl. (2) die entsprechenden Geschwindigkeitskonstanten $k_{3}^{\text {NMR }}$ berechnet [Gl. (2): $k_{3}^{\text {NMR }}=(\ln 2) \tau_{1 / 2}{ }^{-1}$ ]. Die Ergebnisse dieser Untersuchungen sind in Tab. 1 zusammengestellt.

c) DSC-Untersuchungen zur Kinetik. - Durchführung der DSCMessungen: Für die Messungen wurden ein Kalorimeter des Typs Heraeus TA 500 (Wärmefluß-Differenzkalorimeter), Heraeus (Deutschland), sowie ein MCB-Mikrokalorimeter (zu einem Lejstungskompensationskalorimeter umgebautes, modifiziertes CALVET-Typ-Kalorimeter ${ }^{[22}$ ), Thermanalyse (Frankreich), verwendet (Details: Lit. ${ }^{[1 d, 11]}$ ). Beide Kalorimeter wurden einer sorgfältigen Wärme- und Temperaturkalibrierung unter Verwendung mehrerer Kalibriermaterialien unterworfen, deren Schmeizenthalpien und -temperaturen genau bekannt sind (vgl. hierzu Lit. ${ }^{[23)}$ ).

Die Silane 1a-16a (pur und/oder als ca. $0.1 \mathrm{M}$ Lösungen; vgl. hierzu Tab. 2 und 3) wurden in hermetisch verschlossenen Alumi- 
niumkapseln (Heraeus TA 500; Silan-Einwaagen zwischen 5 und $10 \mathrm{mg}$ ) bei Heizraten von 1 bis $10 \mathrm{~K} \mathrm{~min}^{-1}$ oder in bis 90 bar druckfesten, abgeschmolzenen Glasampullen (MCB; Silan-Einwaagen zwischen 30 bis $50 \mathrm{mg}$, z.T. in Lösung) bei Heizraten von 0.3 und $0.5 \mathrm{~K} \mathrm{~min}^{-1}$ vermessen. Das Befüllen der Aluminiumkapseln („Glovebox“) und Glasampullen (Vakuum) erfolgte unter Feuchtigkeitsausschluß. Das durchfahrene Temperaturintervall lag zwischen 20 und $270^{\circ} \mathrm{C}$. Der jeweilige Vergleichsprobenbehälter war leer. Aufgezeichnet wurde der Wärmestrom $\mathrm{d} q / \mathrm{d} t$ als Funktion der Zeit und der Temperatur. Zusätzlich zur Meßkurve wurde jeweils die Grundlinie unter den gleichen Bedingungen, jedoch ohne Edukt (d.h. meistens mit der zu Ende reagierten Probe) aufgenommen.

Auswertung der DSC-Messungen: Die Auswertung der DSC-Kurven erfolgte nach dem in Lit. ${ }^{[1 d, 11]}$ beschriebenen Algorithmus. Die Geschwindigkeitskonstante $k_{\mathrm{i}}$ wurde nach der Methode von Borchardt und Daniels ${ }^{[9]}$ - unter Annahme einer Reaktionsordnung von $n=1$ - ermittelt.

Umsatz $\alpha_{i}$ und Umsatzgeschwindigkeit $\mathrm{d} \alpha_{i} / \mathrm{d} t$ zu einer bestimmten Zeit $t_{i}$ berechnen sich aus der jeweils freigesetzten Teilreaktionsenthalpie $\Delta h_{i}$ und dem dabei freigesetzten Reaktionswärmestrom $\mathrm{d} H_{i} / \mathrm{d} t[\mathrm{Gl} .(3,4)]$ :

$$
k_{i}=A \exp \left(-\frac{E_{\mathrm{A}}}{\mathrm{R} T_{i}}\right)=\frac{\frac{\mathrm{d} \alpha_{i}}{\mathrm{~d} t}}{\left(1-\alpha_{i}\right)}=\frac{\frac{\mathrm{d} H_{\mathrm{i}}}{\mathrm{d} t}}{\Delta_{\mathrm{r}} H\left(1-\frac{\Delta h_{i}}{\Delta_{\mathrm{r}} H}\right)}
$$

mit

$$
\Delta h_{i}=\sum_{j=i_{\text {stor }}}^{i}\left(\frac{\mathrm{d} H_{j}}{\mathrm{~d} t}\right) \Delta t
$$

Aus dem linearen Zusammenhang zwischen $\ln \left(k_{i}\right)$ und $1 / T_{i}(\mathrm{Ar}-$ rhenius-Darstellung) ${ }^{[24 \mid}$ lassen sich mit Hilfe der Linearen Regression $\lg (A)$ und $E_{\mathrm{A}}$ bestimmen. Durch Multiple Lineare Regression ${ }^{[2]}$ können $n, \lg (A)$ und $E_{\mathrm{A}}$ zugleich erhalten werden [Gl. (5)]:

$$
\ln \left(\frac{\mathrm{d} \alpha}{\mathrm{d} t}\right)=\ln (A)-\frac{E_{\mathrm{A}}}{\mathrm{R}} \frac{1}{T}+n \ln (1-\alpha)
$$

Aktivierungsenthalpie $\Delta H_{\bar{T}}^{\ddagger}$ und Aktivierungsentropie $\Delta S_{T}^{\ddagger}$ können unter Verwendung der Eyring-Funktion ${ }^{[26]}$ aus Frequenzfaktor $A$ und Aktivierungsenergie $E_{\mathrm{A}}$ berechnet werden $[\mathrm{Gl} .(6,7)]$ :

$$
\begin{aligned}
& \Delta H_{T}^{\ddagger} \approx E_{\mathrm{A}}-\mathrm{R} T \\
& \Delta S_{T}^{\neq}=\mathrm{R}\left[\ln (A)-\ln (T)-\ln \left(\frac{\mathrm{k}_{\mathrm{B}}}{\mathrm{h}}\right)-1\right]
\end{aligned}
$$

(k $\mathrm{k}_{\mathrm{B}}$ : Boltzmann-Konstante, h: Planck-Konstante)

Die Gibbssche Aktivierungsenergie $\Delta G_{\neq}^{\neq}$und die Geschwindigkeitskonstante $k_{T}$ erhält man aus $\lg (A)$ und $E_{\mathrm{A}}[\mathrm{Gl} .(8,9)]$ :

$$
\begin{aligned}
\Delta G^{\ddagger} & =\Delta H_{F}^{*}-T \Delta S^{\ddagger} \\
k_{T} & =A \exp \left(-\frac{E_{\mathrm{A}}}{\mathrm{R} T}\right) .
\end{aligned}
$$

Die Ergebnisse dieser Untersuchungen sind in Tab. $1-3$ zusam. mengestellt, wobei die MeBwerte in der Form $\bar{x}=\bar{x} \pm \sigma_{x}$ ange- geben sind. Die Berechnung des Mittelwerts $\bar{x}$ und des mittleren Fehler des Mittelwerts $\sigma_{\varepsilon}$ aus $n$ voneinander unabhängigen Einzelmessungen $x_{i}$ erfolgte nach $[\mathrm{Gl} .(10,11)]$ :

$$
\begin{aligned}
\bar{x} & =\frac{1}{n} \sum_{i=1}^{n} x_{i} \\
\sigma_{\ddot{x}} & =\sqrt{\frac{1}{n(n-1)} \sum_{i=1}^{n}\left(x_{i}-\bar{x}\right)^{2}}
\end{aligned}
$$

\section{d) Theoretische Untersuchungen}

Zur Berechnung der Moleküleigenschaften von 21 a und 21 b wurden das semiempirische MNDO-Verfahren ${ }^{[12]}$ und das ab-initioPaket GAUSSIAN $82^{[13]}$ benutzt, wobei die ab-initio-Rechnungen auf dem Hartree-Fock-Niveau vorgenommen wurden. Die Rechnungen wurden auf Anlagen des Typs Amdahl 470/Vb (MNDO) und des Typs Cray X-MP/24 (GAUSSIAN 82) durchgeführt.

Ausgehend von einer Startgeometrie mit Standardbindungsabständen ${ }^{[14]}$ und Bindungswinkeln, die der jeweiligen Hybridisierung der Atome entsprechen, wurden dic Geometrien von 21 a und 21 b mit Hilfe des MNDO-Verfahrens optimiert. Die so erhaltenen Geometrien wurden mit Hilfe einer ab-initio-Rechnung mit minimalem Basissatz (STO-3G) reoptimiert. Die Berechnung der Energien erfolgte mit einem "double-zeta“-Basissatz unter Mitnahme von Polarisationsfunktionen an den Nichtwasserstoff-Atomen (6-31 G*-Basissatz), wobei das Kerngerüst festgehalten wurde.

Die Geometrie des Übergangszustands wurde bei Kenntnis der Geometrie von Anfangs- (21 a) und Endzustand (21 b) auf MNDONiveau mit Hilfe eines Algorithmus nach Dewar ${ }^{[27]}$ berechnet. Die so erhaltene Aktivicrungsenthalpic lag im Vergleich zu den experimentell bestimmten Größen für die Umlagerungen von 1a-16a um den Faktor vier zu hoch. Um den ungewöhnlichen Bindungslängen des Ùbergangszustands gerecht zu werden, wurde bei der Reoptimierung der Geometrie mit Hilfe des ab-initio-Verfahrens ein Basissatz benutzt, bei dessen Valenzorbitalen langreichwcitige und kurzreichweitige Anteile unabhängig voneinander variiert werden konnten (3-21G-Basissatz). Der Zustand geringster Energie mit einem negativen Eigenwert der Hessschen Matrix ${ }^{[28]}$ wurde als Übergangszustand definiert; dieser zerfiel im Verlaufe weiterer Optimierung stets in den Endzustand (21 b). Für die so berechnete Geometrie des Úbergangszustands (vgl. Schema 16) wurde die Bildungsenergie mit einem $6-31 \mathrm{G}^{*}$-Basissatz berechnet.

\section{CAS-Registry-Nummern}

1a: $84802-95-9$ / 1b: 2754-27-0 / 2a: 88811-67-0 / 2b: 17887-60-4 3a: 88811-68-1 / 3b: 17909-07-8 / 4a: 137847-26-8 / 4b: 18407 49-3 / 5a: 122887-66-5 / 5b: 71958-71-9 / 6a: 122887-65-4 / 6b: 5089-53-2 / 7a: 122908-71-8 / 7b: 122887-74-5 / 8a: 137847-27-9 8b: 137847-48-4 /9a: 137847-28-0/9b: 81851-79-8 / 10a: 137847 29-1 / 10b: 137847-49-5 / 11a: 99542-57-1 /[D]-11a: 122887-71-2 / 11 b: 27607-77-8 / [D]-11 b: 122887-72-3/12a: 99542-58-2 / 12 b: 99542-59-3 / 13a: 122887-63-2 / 13b: 91158-37-1 / 14a: 137847 . 30-4 / 14b: 18789-78-1 / 15 a: 122887-67-6 / 15b: 122887-75-6 / 16a 137847-31-5 / 16b: 137847-50-8 / 17a: 122887-70-7 / 17c: 1833 31-4 / 18a: 122887-68-7 / 18c: $13683-11-9$ / 19a: $122887-69-8$ / 19c 110881-29-3 / 20a: 122908-72-9 / 20c: $25261-14-7$ / 21 a: 137847 32-6 / 21 b: 137847-51-9 / 22: 2212-11-5 / 23: 137847-33-7 / 24 137847-34-8 / 25: 137847-35-9 / 26: 1558-33-4 / 27: 66817-66-1 28: 137847-36-0 / 29: 137847-37-1 / 30: 123278-41-1 / 31: 137847 38-2 / 32: 137847-39-3 / 33: 137847-40-6 / 34: 137847-41-7 / 35: 137847-42-8 / 36: 137847-43-9 / 37: 8444-50-6 / 38: 18230-72-3 [D]-38: 137847-52-0 / 39: 18420-19-4/40: 137847-44-0/41: 137847-45-1 / 42: 137847-46-2 / 43: 137847-47-3 
${ }^{[1]}{ }^{11 \mathrm{a}]}$ R. Tacke, H. Lange, Chem. Ber. 1983, 116, 3685-3691. [1b] R. Tacke, M. Link, A. Bentlage-Felten, H. Zilch, Z. Naturforsch., Teil B, 1985, 40, 942-947. - [1c] R. Tacke, M. Link, H. Zilch, Chem. Ber. 1985, 118, 4637-4640. - [1d] S. Sarge, H. K. Cammenga, B. Becker, R. Rohr-Aehle, R. Tacke, J. Thermal Analysis 1988, 33, 1185-1192. - [1e] R. Tacke, R. Rohr-Aehle, J. Organomet. Chem. 1988, 354, 139-146.

${ }^{[2]}$ R. Tacke, J. Sperlich, C. Strohmann, G. Mattern, Chem. Ber. 1991, 124, 1491 - 1496.

${ }^{[3]}$ R. Tacke, H. Lange, A. Bentlage, Chem. Ber. 1982, 115, 3673-3677.

[4] L. H. Sommer, R. M. Murch, F. A. Mitch, J. Am. Chem. Soc. 1954, 76, 1619-1621.

[5] [Sa] E. W. Abel, I. H. Sabherwal, J. Chem. Soc. A 1968

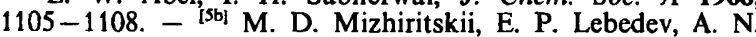
Fufaeva, Zh. Obshch. Khim. 1982, 52, 2089-2092; J. Gen. Chem. 1982, 52, 1859-1862.

${ }^{[6]}$ H. Gilman, K. Shiina, D. Aoki, B. J. Gaj, D. Wittenberg, T. Brennan, J. Organomet. Chem. 1968, 13, 323-328.

[7] M. Kumada, J.-I. Nakajima, M. Ishikawa, Y. Yamamoto, J. Org. Chem. 1958, 23, 292-295.

${ }^{\text {[8] }}$ Mit dieser Methode kann der Umsatz $\propto$ einer Reaktion über die ihm proportionale Teilreaktionsenthalpie $\Delta h$ als Funktion der Temperatur $T$ und zugleich der Zeit $t$ (zeitlineares Temperaturprogramm) verfolgt werden. Aus der erhaltenen Meßkurve lassen sich im Idealfall alle gesuchten Größen (Reaktionsordnung. Frequenzfaktor, Aktivierungsenergie, Reaktionsenthalpie) bestimmen.

[9] H. J. Borchardt, F. Daniels, J. Am. Chem. Soc. 1957, 79, 41-46.

${ }^{[10]} \mathrm{Da}$ DSC-Messungen, bei denen vor Versuchsbeginn bereits ein Teil des Edukts reagiert hat, zwar noch verläßliche kinetische Parameter liefern, aber für die Berechnung der Reaktionsenthalpie unbrauchbar sind, findet man in Tab. 2 und 3 für $j$ und $i$ teilweise unterschiedliche Werte.

[11] S. Sarge, Dissertation, Technische Universität Braunschweig, 1988.

[12] M. J. S. Dewar, W. Thiel, J. Am. Chem. Soc. 1977, 99, $4899-4907$.

[13] W. J. Hehre, C. Radom, P. v. R. Schleyer, J. A. Pople, Ab Initio Molecular Orbital Theory. Wiley-Interscience, New York, 1985.

${ }^{[14]}$ T. Clark, A Handbook of Computational Chemistry, John Wiley \& Sons, New York, 1985

[15] Übersichtsarbeit: [15a] A. G. Brook, A. R. Bassindale, in Rearrangements in Ground and Excited States (Hrsg.: P. de Mayo), Academic Press, New York, 1980, S. 149-227. - Originalarbeiten: ${ }^{[15 b]}$ M. Kumada, M. Ishikawa, K. Tamao, J. Organomet. Chem. 1966, 5, 226-232. - ${ }^{[15 c]}$ A. G. Brook, P. F. Jones, Chem Commun. 1969, 1324-1325. - [15d] W. I. Bevan, R. N. Haszeldine, J. Middleton, A. E. Tipping, J. Chem. Soc. Dalton Trans. 1975, 252-256. - [ise] A. R. Bassindale, A. G. Brook, P. F. Jones, J. M. Lennon, Can. J. Chem. 1975, 53, 332-337. - [1si]
L. S. Zakharov, T. D. Drozdova, V. A. Syoren, L. L. Morozov, M. I. Kabachnik, Izv. Akad. Nauk SSSR, Ser. Khim. 1977, 644-647; Bull. Acad. Sci. USSR, Div. Chem. Sci. (Engl. Transl.)

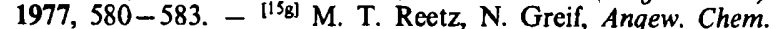
1977, 89, 765-766; Angew. Chem. Int. Ed. Engl. 1977, 16, 712-713. - [15h] J. D. Buynak, J. B. Strickland, T. Hurd, A. Phan, J. Chem. Soc., Chem. Commun. 1989, 89-90.

[16] Neben den von uns bereits veröffentlichten Beispielen für diesen Reaktionstyp (vgl. hierzu Lit. ${ }^{(i)}$ ) sei auch auf Untersuchungen zu der radikalisch initiierten Umlagerung $1 \mathbf{a} \rightarrow \mathbf{1}$ b in Lösung (vgl. hierzu Lit. ${ }^{[20]}$ und auf Studien zu der thermisch induzierten Umlagerung von (Chlormethyl)diorganylhydridosilanen in der Gasphase (vgl. hicrzu Lit. $\left.{ }^{[1]}\right)$ hingewiesen. Die in Lit. ${ }^{[20,21]}$ beschriebenen Umlagerungen sind jedoch mechanistisch nicht mit dem von uns untersuchten Reaktionstyp vergleichbar.

[17) Die hier angewandte nichtisotherme Reaktionsanalyse (DSC) bietet gegenüber der isothermen Bestimmung der Reaktionsgeschwindigkeit durch NMR-Messungen Vorteile, da im Prinzip alle kinetischen und energetischen Parameter einer Reaktion mit einer einzigen Messung bestimmt werden können (von Messung zu Messung schwankende äußere Versuchsbedingungen haben keinen störenden Einfluß auf die Ergebnisse; geringer Substanzbedarf; Zeitersparnis).

[18] Jeweils $300 \mu \mathrm{l}$ einer 5proz. Lösung von 9a in Toluol wurden $69 \mathrm{~h}$ auf $80^{\circ} \mathrm{C}$ erhitzt. Bei einem Druck von 1 bar war kein Umlagerungsprodukt nachweisbar; bei einem Druck von 8.2 kbar wurde ein 40proz. Umsatz zu 9b nachgewiesen ('H-NMR).

[19] Den ${ }^{29} \mathrm{Si}$-NMR-Spektren der Edukte in Lösung (vgl. hierzu den exp. Teil) konnten keine Anhaltspunkte für eine Pentakoordination des Siliciums im Grundzustand entnommen werden. Gleiches gilt für 9a im Kristall (Einkristall-Röntgenstrukturanalyse; H. Goesmann, R. Tacke, F. Wiesenberger, unveröffentlichte Ergebnisse).

[20] J. Wilt, S. M. Keller, J. Am. Chem. Soc. 1983, 105, 1395-1396.

[21] [21a] J. G. Martin, M. A. Ring, H. E. O'Neal, Organometallics 1986, 5, 1228-1230. - [16] I. M. T. Davidson, S. Ijadi-Maghsoodi, Organometallics 1986, 5, 2086-2090. - ${ }^{[21 c]}$ M. P. Clarke, R. Damraur, I. M. T. Davidson, R. Simon, Organometallics 1989, 8, 2031-2033.

[22] J. Reichelt, Dissertation, Technische Universität Braunschweig, 1986.

${ }^{[23]}$ S. Sarge, H. K. Cammenga, Thermochim. Acta 1985, 94, 17-31.

${ }^{[24]}$ S. Arrhenius, Z. Phys. Chem. (Leipzig) 1889, 4, 225-248.

[25] H. Wyden, G. Widman, in Experientia Suppl. Vol. 37 (Hrsg.: E. Marti, H. R. Oswald, H. G. Wiedemann), Birkhäuser Verlag, Basel, 1979, S. 284-290.

${ }^{[26]}$ H. Eyring, Trans. Faraday Soc. 1938, 34, 41-48.

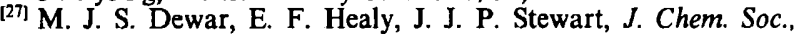
Faraday Trans. 2, 1984, 80, 227-233.

[28] J. D. Head, B. Weiner, M. C. Zerner, Int. J. Quantum Chem. 1988, 33, $177-186$.

[344/91] 